\title{
The effects of oar-shaft stiffness and length on rowing biomechanics
}

Brock Laschowski, The University of Western Ontario

Supervisor: Dr. Volker Nolte, The University of Western Ontario

A thesis submitted in partial fulfillment of the requirements for the Master of Science degree in Kinesiology

(C) Brock Laschowski 2014

Follow this and additional works at: https://ir.lib.uwo.ca/etd

Part of the Biomechanical Engineering Commons, Biomechanics Commons, and the Sports Sciences Commons

\section{Recommended Citation}

Laschowski, Brock, "The effects of oar-shaft stiffness and length on rowing biomechanics" (2014).

Electronic Thesis and Dissertation Repository. 2270.

https://ir.lib.uwo.ca/etd/2270

This Dissertation/Thesis is brought to you for free and open access by Scholarship@Western. It has been accepted for inclusion in Electronic Thesis and Dissertation Repository by an authorized administrator of Scholarship@Western. For more information, please contact wlswadmin@uwo.ca. 


\section{THE EFFECTS OF OAR-SHAFT STIFFNESS AND LENGTH ON ROWING BIOMECHANICS}

(Thesis format: Integrated Article)

by

Brock Laschowski

Graduate Program in Kinesiology

A thesis submitted in partial fulfillment

of the requirements for the degree of

Masters of Science

The School of Graduate and Postdoctoral Studies

The University of Western Ontario

London, Ontario, Canada

(C) Brock Laschowski 2014 


\begin{abstract}
This work investigates the effects of oar-shaft stiffness and length on rowing biomechanics. The mechanical properties of the oar-shafts were examined using an end-loaded cantilever system, and theoretical relations were proposed between the mechanics of the oar-shafts and rowing performance. On-water experiments were subsequently conducted and rowing biomechanics measured via the PowerLine Rowing Instrumentation System. The PowerLine system measures force and oar angle on the oarlock, as well as proper boat acceleration. The convergent validity and test-retest reliability of the PowerLine force measurements were determined prior to the on-water experiments.

Thereafter, rowers were tested over a set distance using oar-shafts of different stiffness and length. There were slight differences in the biomechanics between rowing with the different oar configurations. However, the measured differences in the biomechanical parameters were on the same order of magnitude as the rower's inter-stroke inconsistencies.
\end{abstract}

\title{
Keywords
}

Rowing, Oar-Shaft, Biomechanics, Stiffness, Flexural Rigidity, Deflection, Cantilever Beam, Oarlock, Strain Gauge, Test-Retest Reliability, Convergent Validity 


\section{Co-Authorship Statement}

This work contains material from manuscripts currently under peer-review (Chapters 2-4). On all manuscripts, Brock Laschowski was the first author, and Dr. Volker Nolte was a co-author. Dr. John de Bruyn and Cameron Hopkins were co-authors of Chapter 2, and Michael Adamovsky and Ryan Alexander were coauthors of Chapter 4. All data presented in this thesis was primarily collected, analyzed and interpreted by Brock Laschowski. 


\section{Acknowledgments}

I want to thank:

- Dr. Volker Nolte, my MSc supervisor

- Dr. John de Bruyn and Cameron Hopkins from the Department of Physics and Astronomy

- Michael Adamovsky and Ryan Alexander from the Faculty of Engineering

- Will George from the Canadian Sport Institute Ontario

- Dr. Daniel Bechard and Matt Waddell from the Western Rowing Program

- Katherine Cornacchia, my fiancé

"Think Different" - Steve Jobs (1955-2011) 


\section{Table of Contents}

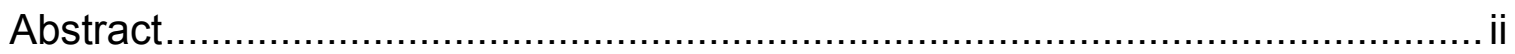

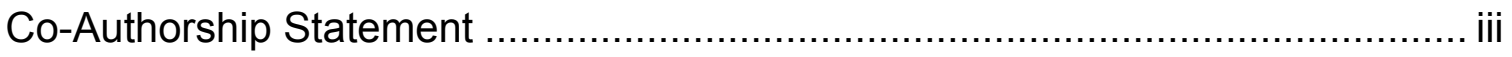

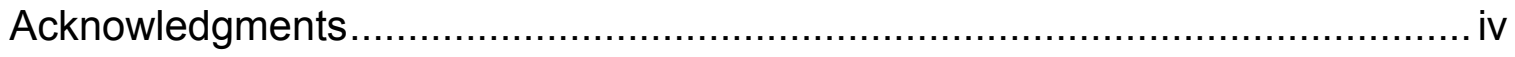

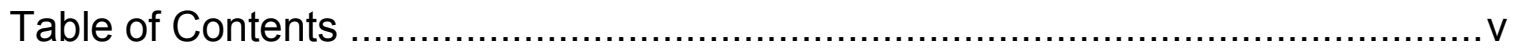

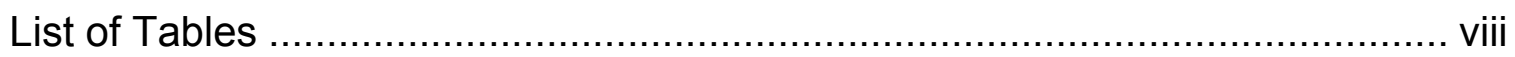

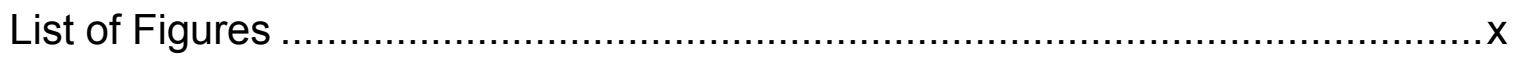

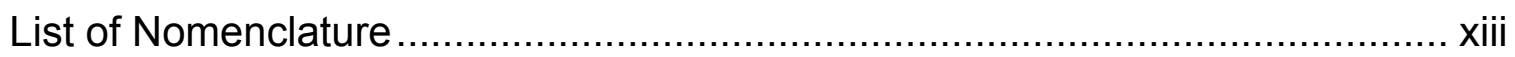

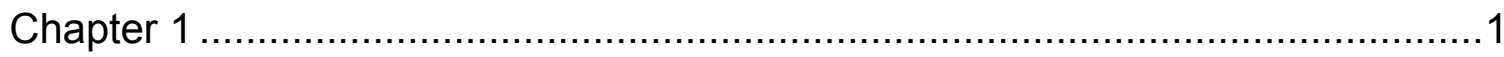

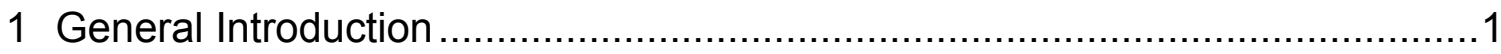

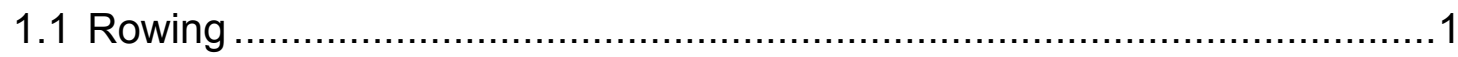

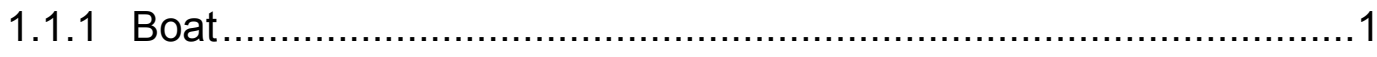

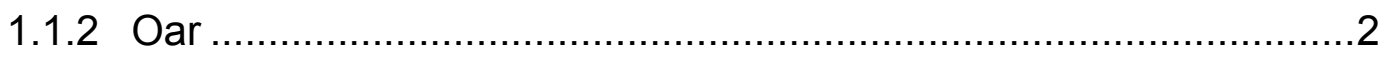

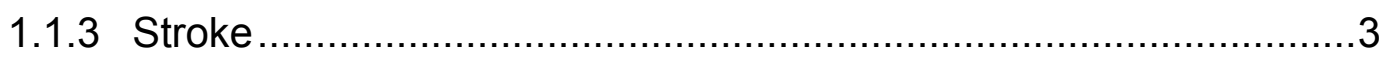

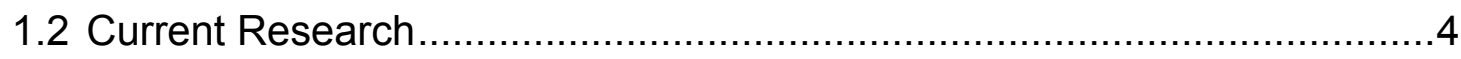

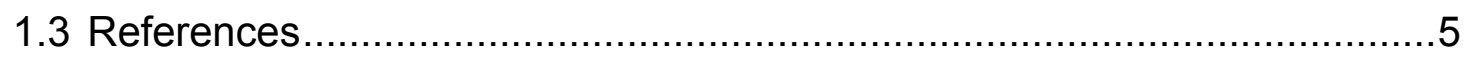

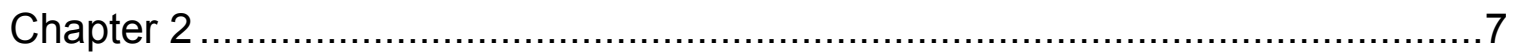

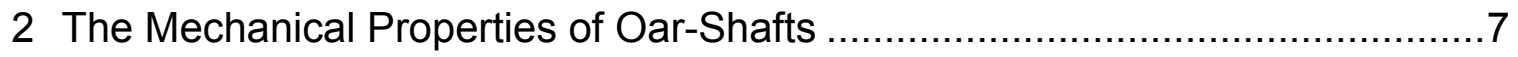

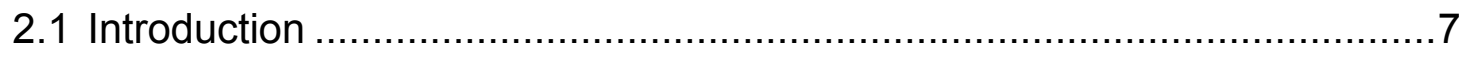

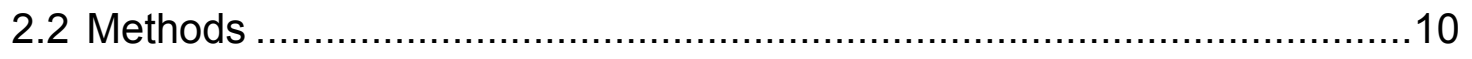

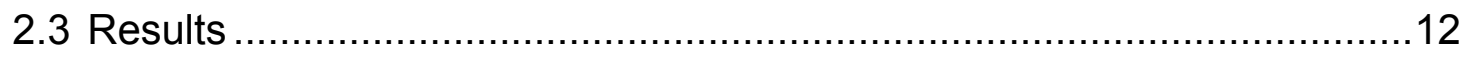

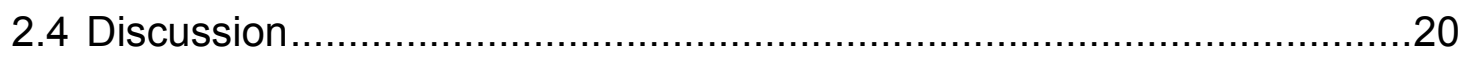

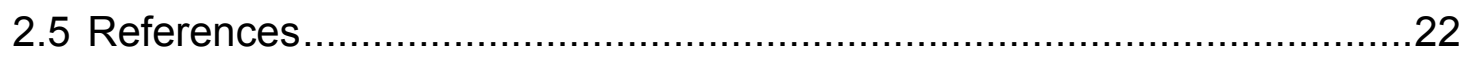

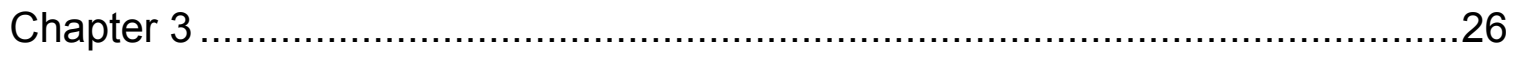


3 Validity and Reliability of the PowerLine Rowing Instrumentation System......26

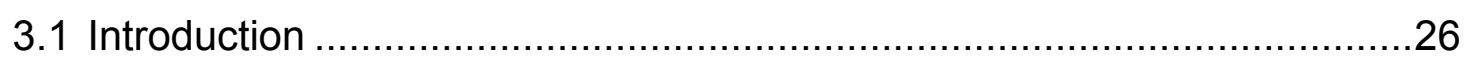

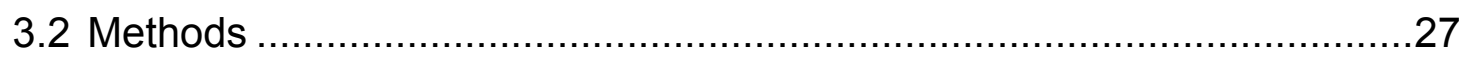

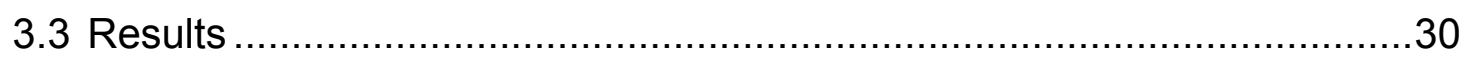

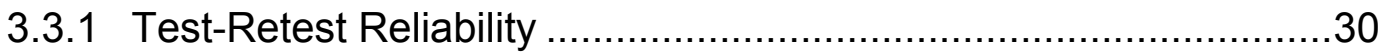

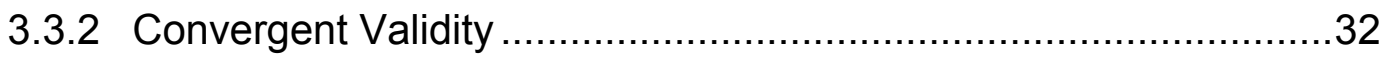

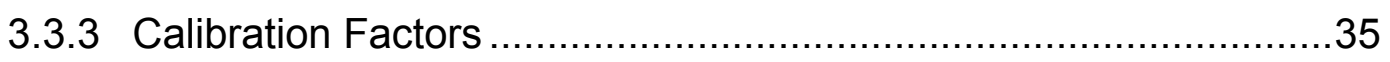

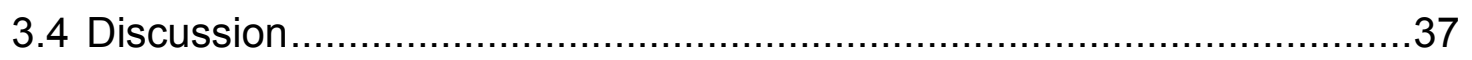

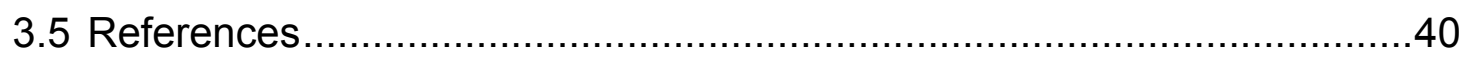

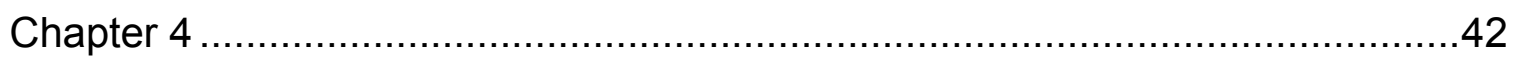

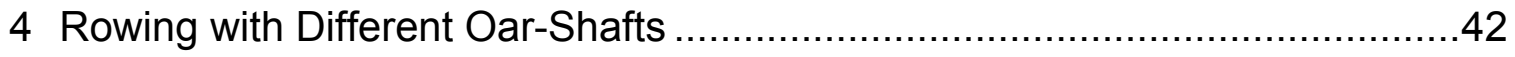

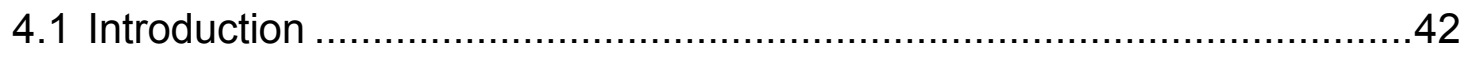

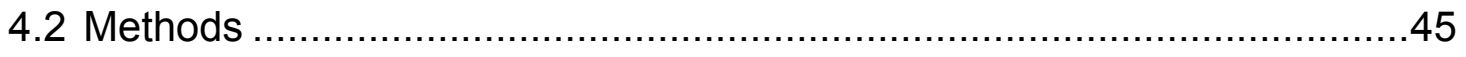

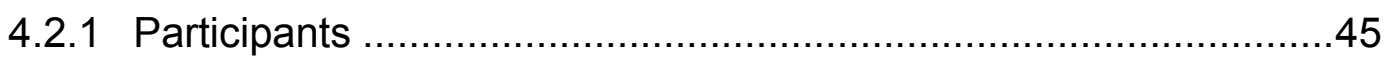

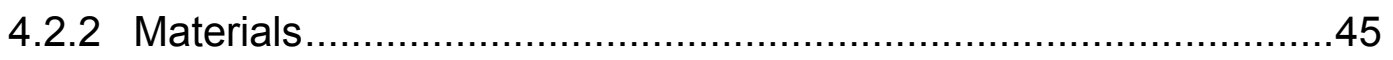

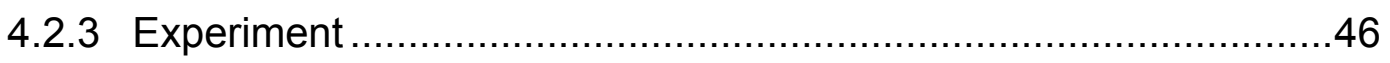

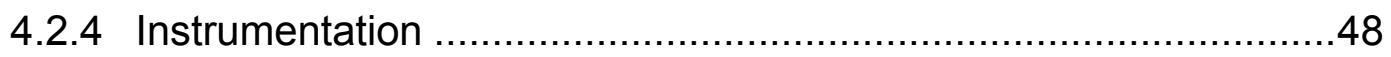

4.2.5 Data Analysis and Signal Processing …...................................49

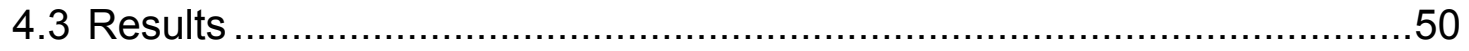

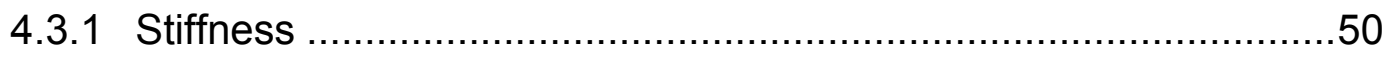

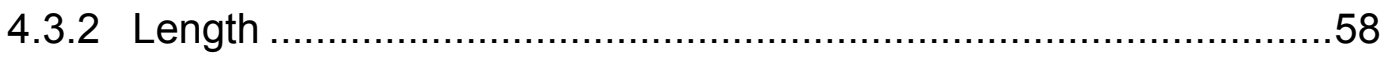

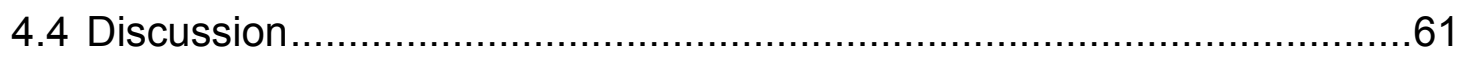

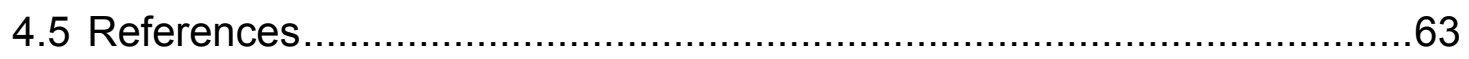

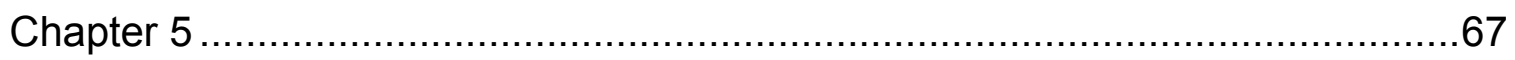

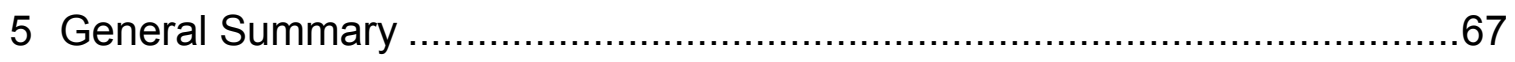




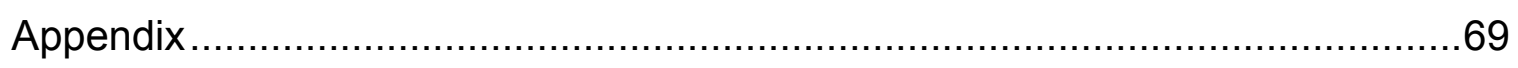

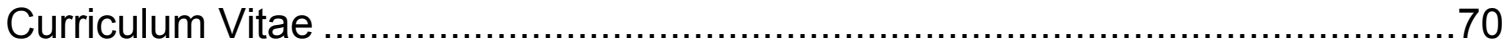




\section{List of Tables}

Table 1. The twelve oar configurations that were investigated. Each configuration is designated by a code that indicates the stiffness ( $M$ or ES), side (P or S), and total length of the oar. The total length of the oar varied by changing $L_{b}$.

Table 2. The flexural rigidity $E I\left(\mathrm{Nm}^{2}\right)$ for each oar configuration at each position $y_{i}$ along the oar-shaft. The uncertainties are SD.

Table 3. The deflection angles $\theta$ at the blade-end of the oar-shafts for each configuration when loaded with $201.04 \mathrm{~N}$. The uncertainties are SD.

Table 4. Analyzing normality of the PL force measurements as a function of the testing date using a Shapiro-Wilk test.

Table 5. Investigating normality of the PL force measurements as a function of the known static forces using a Shapiro-Wilk test

Table 6. Testing the convergent validity of the PL force measurements using a Wilcoxon One-Sample Signed Rank Test.

Table 7. The slope, $y$-intercept and $\mathrm{R}^{2}$ for the $\mathrm{PL}$ force measurements as a function of the known static forces from a least-squares linear regression analysis.

Table 8. The six oar configurations that were tested. Each configuration is designated by a code that indicates the stiffness ( $M$ or ES) and total length of the oar. The total length of the oar varied by changing the outboard length.

Table 9. The mean stroke rates (strokes/min) during each trial for each rower; the uncertainties are SD. The experiment started with trial 1 and ended with trial 6 . The mean $x$ stroke rate for each rower across all six trials is also provided.

Table 10. Testing the six oar configurations in a different order for each rower. .47 
Table 11. Average wind velocity $(\mathrm{m} / \mathrm{s})$ measured along the testing course during

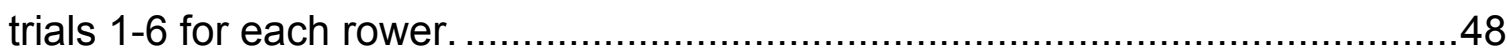

Table 12. The maximum force $(\mathrm{N}), \%$ of the drive to maximum force, and impulse (Ns) measured on port and starboard oarlocks for each oar configuration for all four rowers. The results are given by the mean \pm SD over 20 strokes...............54

Table 13. $200 \mathrm{~m}$ performance time (s) for each rower for each trial. The mean $x$ performance time for each rower across all six trials is also provided..............55 


\section{List of Figures}

Figure 1. Schematic of the rowing boat from an aerial view. The components of the boat are discussed in the text.

Figure 2. Schematic of the rowing oar. The properties of the oar are discussed in the text. 3

Figure 3. The locations of the seat and oars at the catch [grey] and finish [black] positions. The oar angles are $0^{\circ}$ when the oars are perpendicular to the boat's main direction of motion. 4

Figure 4. Photograph of a sweep oar-shaft during on-water rowing with visible deflection.

Figure 5. Schematic of the setup used to measure the oar-shaft's deflection from its equilibrium position $E_{x}$ at six positions $y_{i}$ along the shaft when a static load $W$ is applied at length $L_{b}$ from Clamp 2. The support length $L_{s}$ is the distance between Clamps 1 and 2. The deflection angle at the blade-end of the oar-shaft is denoted by $\theta$. 10

Figure 6. Photograph of the experimental set-up used to measure oar-shaft deflection. The oar is secured to a laboratory bench with a custom-made support stand, and a weight plate is suspended from the oar-shaft using a tether.

Figure 7. Deflection $\delta$ as a function of position $y$ for MP2.70 and ESP2.70 when loaded with $111.8 \mathrm{~N}$. The curves are fits to equation (1)

Figure 8. Deflection $\delta$ as a function of load $W$ for MP2.66 at three positions $y_{i}$ along the oar-shaft. The lines are fits to the data discussed in the text.

Figure 9. Flexural rigidity El as a function of position $y$ for MP2.70 and ESP2.70. 
Figure 10. Flexural rigidity $E l$ as a function of position $y$ for port and starboard versions of Oar $\mathrm{M}$. The three data points in each group correspond to oar lengths of $2.66,2.68$, and $2.70 \mathrm{~m}$, as shown by the sketched curves

Figure 11. Deflection angles $\theta$ at the blade-end of the oar-shafts for MP2.70 and ESP2.70 as a function of load $W$. The linear fits are expressions to equation (3).

Figure 12. Orientating the PL swivel perpendicular to the base of the inner tube (shown in the left side of the photograph) using a spirit level.

Figure 13. Photograph of the experimental setup used to test the PL oarlocks. A bar is supported by two stands, and a PL oarlock is fixed to the bar. The PL swivel is pointed in the $x$-direction and the base of the inner tube is pointed in the $y$-direction. The PL oarlock is connected to a data-logger, and a suspension rig is hanging from the oarlock.

Figure 14. The PL force measurements as a function of the known static forces for a single sculling oarlock. The linear fit is a regression line.

Figure 15. Schematic of the oar-shaft's dynamic behavior during the drive. The equilibrium position $E_{x}$ refers to the point where the magnitude of the oar-shaft's deflection in the $x$-axis is zero. $\delta$ is deflection and $\delta^{-1}$ is inverse deflection, as described in the text. Deflection of the inboard is neglected in this model.

Figure 16. Free body diagram of the external forces that act on the rowing oar during the drive. $F_{h}$ is the force applied by the rower to the handle, $F_{\mathrm{o}}$ is the normal reaction force at the oarlock, $M$ is the resultant moment of force, $F_{b}$ is the load on the blade, $L_{b}{ }^{\prime}$ is the beam moment arm, and $L_{s}{ }^{\prime}$ is the support moment arm.

Figure 17. Boat acceleration as a percentage of the drive between ES2.70 and M2.70 for rower 1 . The fits to the data are smoothing splines. 
Figure 18. Boat acceleration as a percentage of the drive between ES2.70 and M2.70 for rower 2. The fits to the data are smoothing splines.

Figure 19. Boat acceleration as a percentage of the drive between ES2.70 and M2.70 for rower 3. The fits to the data are smoothing splines.

Figure 20. Boat acceleration as a percentage of the drive between ES2.70 and M2.70 for rower 4 . The fits to the data are smoothing splines.

Figure 21. Oarlock force as a function of angle between ES2.70 and M2.70 for rower 1 on port side. The fits are smoothing splines.

Figure 22. Oarlock force as a function of angle between ES2.70 and M2.70 for rower 2 on port side. The fits are smoothing splines.

Figure 23. Oarlock force as a function of angle between ES2.70 and M2.70 for rower 3 on port side. The fits are smoothing splines.

Figure 24. Oarlock force as a function of angle between ES2.70 and M2.70 for rower 4 on port side. The fits are smoothing splines.

Figure 25. Oarlock force as a function of angle between M2.66 and M2.70 for rower 1 on port side. The fits are smoothing splines.

Figure 26. Oarlock force as a function of angle between M2.66 and M2.70 for rower 2 on port side. The fits are smoothing splines.

Figure 27. Oarlock force as a function of angle between M2.66 and M2.70 for rower 3 on port side. The fits are smoothing splines.

Figure 28. Oarlock force as a function of angle between M2.66 and M2.70 for rower 4 on port side. The fits are smoothing splines. 


\section{List of Nomenclature}

\begin{tabular}{|c|c|}
\hline AOA & Angle of attack $\left({ }^{\circ}\right)$ \\
\hline$M$ & Medium oar-shaft \\
\hline ES & Extra-Soft oar-shaft \\
\hline$y_{i}$ & Position on the oar-shaft in the $y$-axis $(\mathrm{m})$ \\
\hline $\boldsymbol{W}$ & Static load $(\mathrm{N})$ \\
\hline$L_{b}$ & Beam length $(\mathrm{m})$ \\
\hline$L_{s}$ & Support length (m) \\
\hline$\theta$ & Angle of deflection $\left({ }^{\circ}\right)$ \\
\hline$\delta$ & Deflection (m) \\
\hline$P$ & Port \\
\hline S & Starboard \\
\hline$E$ & Young's modulus $\left(\mathrm{N} / \mathrm{m}^{2}\right)$ \\
\hline I & Area moment of inertia $\left(\mathrm{m}^{4}\right)$ \\
\hline El & Flexural rigidity $\left(\mathrm{N} \cdot \mathrm{m}^{2}\right)$ \\
\hline $\mathrm{PL}$ & PowerLine \\
\hline$R_{e}$ & Electrical resistance $(\Omega)$ \\
\hline$\rho$ & Electrical resistivity $(\Omega \cdot \mathrm{m})$ \\
\hline$L_{g}$ & Length of strain gauge $(\mathrm{m})$ \\
\hline$A$ & Cross-sectional area $\left(\mathrm{m}^{2}\right)$ \\
\hline $\mathrm{R}^{2}$ & Coefficient of determination \\
\hline$H_{0}$ & Null hypothesis \\
\hline SD & Standard deviation \\
\hline$E_{x}$ & Equilibrium position of oar-shaft in $x$-axis \\
\hline $\mathbf{F}_{\mathbf{I}}$ & Lift force $(\mathrm{N})$ \\
\hline $\mathbf{F}_{\mathbf{d}}$ & Drag force $(\mathrm{N})$ \\
\hline$C_{l}$ & Lift coefficient \\
\hline$C_{d}$ & Drag coefficient \\
\hline $\boldsymbol{v}$ & Velocity (m/s) \\
\hline
\end{tabular}




$\begin{array}{ll}A^{\prime} & \text { Reference area }\left(\mathrm{m}^{2}\right) \\ \boldsymbol{\alpha} & \text { Angular acceleration }\left(\mathrm{rad} / \mathrm{s}^{2}\right) \\ \rho_{\mathrm{w}} & \text { Density of water }\left(\mathrm{kg} / \mathrm{m}^{3}\right) \\ \mathrm{F}_{\text {diff }} & \text { Difference in force }(\mathrm{N}) \\ \text { ANOVA } & \text { Analysis of variance } \\ L_{b}{ }^{\prime} & \text { Beam moment arm }(\mathrm{m}) \\ L_{s}^{\prime} & \text { Support moment arm }(\mathrm{m}) \\ \mathbf{F}_{\mathbf{h}} & \text { Handle force }(\mathrm{N}) \\ \mathbf{F}_{\mathbf{b}} & \text { Blade force }(\mathrm{N}) \\ \mathbf{F}_{o} & \text { Oarlock force }(\mathrm{N}) \\ \boldsymbol{M} & \text { Moment of force }(\mathrm{N} \cdot \mathrm{m}) \\ I_{m} & \text { Mass moment of inertia }\left(\mathrm{kg} \cdot \mathrm{m}^{2}\right) \\ \mathrm{F}_{\max } & \text { Maximum force }(\mathrm{N}) \\ \mathrm{F}_{\max \%} & \text { Percentage of drive to } \mathrm{F}_{\max }(\%) \\ r & \text { Pearson product-moment correlation coefficient } \\ \boldsymbol{\delta}^{-1} & \text { Inverse deflection }(\mathrm{m}) \\ \overline{\boldsymbol{x}} & \text { Arithmetic mean } \\ \boldsymbol{x}, y, z & \text { Three-dimensional Cartesian coordinate system } \\ \mathrm{pp} & \text { Percentage point }\end{array}$




\section{Chapter 1}

\section{General Introduction}

\subsection{Rowing}

The main objective in competitive rowing is for a rower to cover a $2000 \mathrm{~m}$ race distance, in a rowing boat, in the least amount of time. Rowing is divided into two classes: sweep and sculling. In sweep, the rower rows with one oar gripped with both hands. There are three types of sweep boats: the pair (i.e., two rowers), the four (i.e., four rowers) and the eight (i.e., eight rowers). In sculling, the rower rows with one oar in each hand simultaneously. There are three types of sculling boats: the single (i.e., one rower), the double (i.e., two rowers) and the quad (i.e., four rowers). The following work will pertain to single sculling unless otherwise specified.

\subsubsection{Boat}

The components of the rowing boat are illustrated schematically in Figure 1. The bow is the end of the boat that leads in the direction of motion, and the stern is the end opposite to the bow. Facing the bow, the left side of the boat is called port and the right side of the boat is called starboard. The three-dimensional Cartesian coordinate system shown in Figure 1, and used throughout this work, is relative to the boat's direction of motion. The $x$-axis is the direction parallel to the boat's main motion (i.e., the stern-bow axis), the $y$-axis is perpendicular to the boat's main motion (i.e., the port-starboard axis) and the $z$-axis is the vertical axis. 


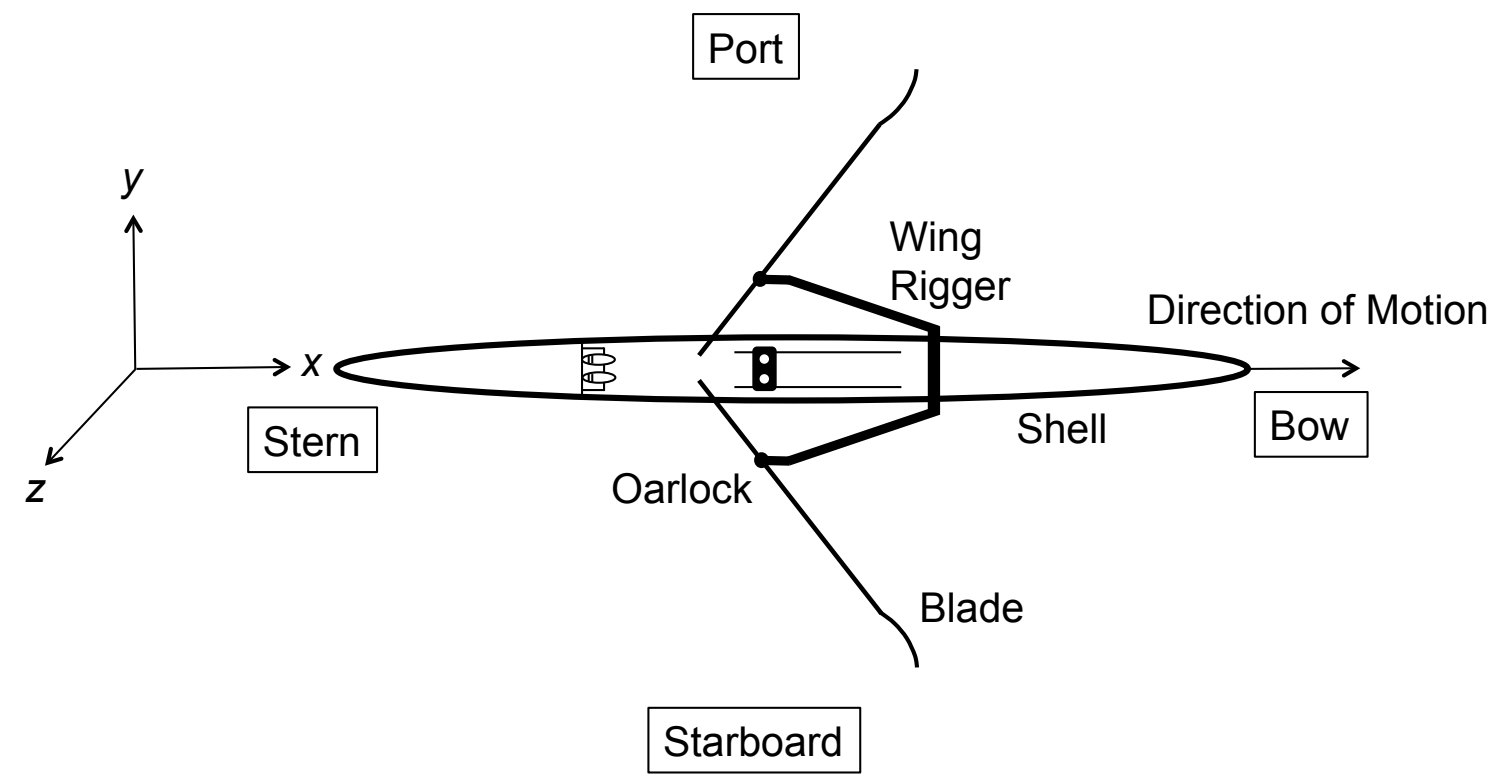

Figure 1. Schematic of the rowing boat from an aerial view. The components of the boat are discussed in the text.

The shell is the supporting structure of the boat that interacts with the water. A wing rigger is mounted on top of the shell, and extends outward from the centerline of the boat in the port and starboard directions. Cylindrical pins at the ends of the wing rigger extend upward in the z-axis. "Oarlocks" slide onto the pins and feature U-shaped channels that are used to support the oars on the wing rigger. Throughout the rowing stroke, the oars rotate with the oarlocks around the pins in the z-axis.

\subsubsection{Oar}

Figure 2 illustrates the components of the rowing oar. The blade is the cleavershaped part of the oar that interacts with the water. The handle is where the rower grips the oar and is located at the opposite end to the blade. The shaft is the long tubular structure between the blade and the handle. A sleeve is attached to the shaft, and a collar is clamped around the sleeve to prevent the oar from translating longitudinally through the oarlock. The outboard length is the distance from the collar to the tip of the blade and the inboard length is the distance from 
the collar to the tip of the handle. The total length of the oar is the sum of the outboard and inboard lengths.

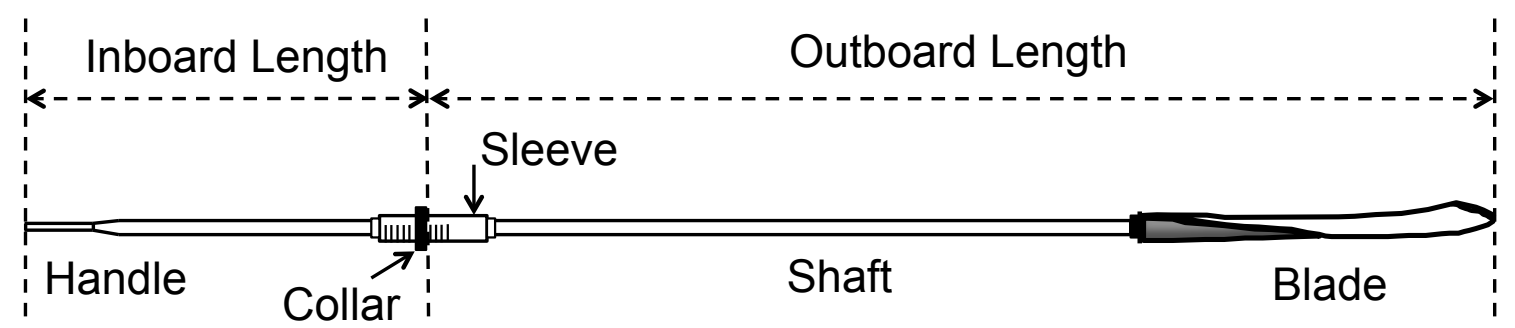

Figure 2. Schematic of the rowing oar. The properties of the oar are discussed in the text.

\subsubsection{Stroke}

The rowing stroke is divided into two positions (i.e., catch and finish) and two phases (i.e., drive and recovery). The oar angles are defined by their rotation around the pins in the z-axis. The catch position is the point where the handles are closest to the stern; it has the highest magnitude of negative oar angle and is defined as the start of the rowing stroke (Figure 3). Following the catch position, the blades enter the water and the rower pulls on the handles. As the handles move towards the bow, the oar angles decrease in negative magnitude. The oar angles are considered to be $0^{\circ}$ when the oars are perpendicular to the boat's main direction of motion [1-2], as illustrated in Figure 3. Passing the perpendicular position, the oar angles increase in positive magnitude as the handles move towards the bow. The finish position is the point in the stroke where the handles are closest to the bow, which has the highest magnitude of positive oar angle. Typical catch and finish angles for an elite heavyweight female sculling rower, averaged over 20 strokes at a rate of 20 strokes $/ \mathrm{min}$, is purportedly $63.2 \pm 1.2^{\circ}$ and $44.0 \pm 0.9^{\circ}$, respectively (Canadian Sport Institute Ontario, 2014, personal communication). The blades are removed from the water slightly before the finish. The drive phase is the motion from the catch to the finish position, and the recovery phase is the motion from the finish position back to the catch. 


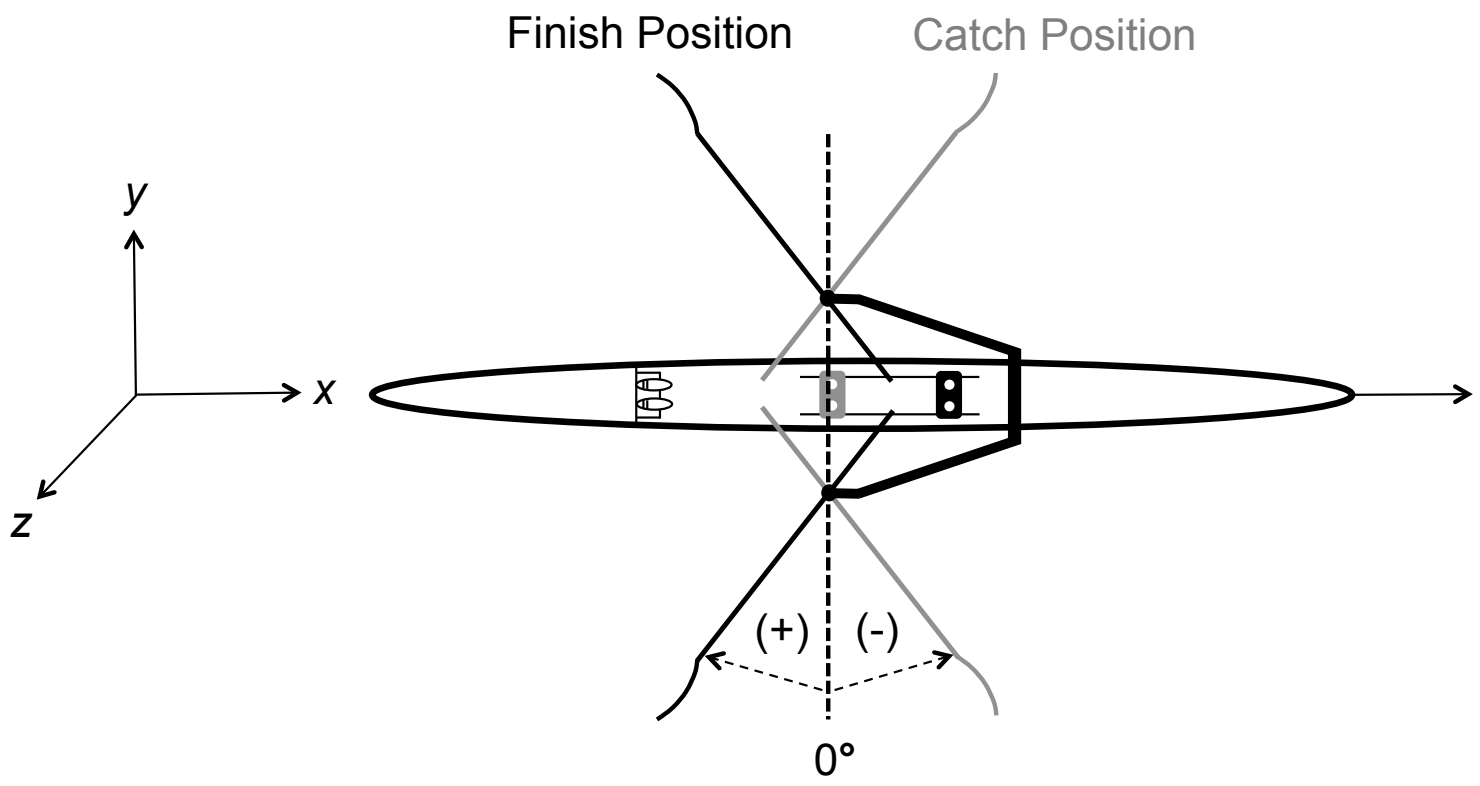

Figure 3. The locations of the seat and oars at the catch [grey] and finish [black] positions. The oar angles are $0^{\circ}$ when the oars are perpendicular to the boat's main direction of motion.

\subsection{Current Research}

Rowing oar-shafts are engineered in a variety of circumferences, lengths, materials and structural designs. However, the effects of these properties on rowing performance are not well known. This is particularly true for oar-shaft stiffness, as many previous studies have assumed that the shaft is perfectly rigid [3-13]. In addition, the effect of oar length on rowing performance is widely discussed. For many years, rower's opted for longer oars because increasing oar length was associated with greater force on the blade [14]. Recently, Nolte [15] presented evidence that suggests shorter oars are more effective in rowing. Previous studies that have considered the effects of oar-shaft stiffness [16, 17] and length [15] on rowing performance have been largely theoretical. In contrast, the following work experimentally investigates the effects of oar-shaft stiffness and length on rowing biomechanics via the PowerLine Rowing Instrumentation System. 


\subsection{References}

1. Dal Monte $A$ and Komor $A$. Rowing and sculling mechanics. In: Vaughan $C L$ (eds) Biomechanics of Sport. USA: CRC Press, 1989, pp.53-119.

2. Nolte V. Die Effektivität des Ruderschlages. [The efficiency of the rowing stroke.] Germany: Bartels and Wernitz, 1984.

3. Affeld K, Schichl K and Ziemann A. Assessment of rowing efficiency. International Journal of Sports Medicine 1993; 14: 39-41.

4. Alexander FH. The theory of rowing. Proceedings of the University of Durham Philosophical Society 1925, pp.160-179.

5. Baudouin A and Hawkins D. A biomechanical review of factors affecting rowing performance. British Journal of Sports Medicine 2002; 36: 396-402.

6. Brearley MN and De Mestre NJ. Modeling the rowing stroke and increasing its efficiency. In: The third Conference on Mathematics and Computers in Sport, Bond University, Queensland, Australia, 30 Sept-2 Oct 1996, pp.35-46.

7. Cabrera D, Ruina A and Kleshnev V. A Simple 1+ Dimensional Model of Rowing Mimics Observed Forces and Motions. Human Movement Science 2006; 25: 192-220.

8. Findlay M and Turnock SR. Mechanics of a rowing stroke: Surge speed variations of a single scull. Proceedings of the Institution of Mechanical Engineers, Part P: Journal of Sports Engineering and Technology 2010; 224: 89100.

9. Hofmijster MJ, Landman EH, Smith RM and Van Soest AJ. Effect of stroke rate on the distribution of net mechanical power in rowing. Journal of Sports Sciences 2007; 25: 403-411. 
10. Macrossan MN and Macrossan NW. Energy efficiency of the rowing oar from catch to square-off. Report, University of Queensland, Australia, May 2008.

11. Sanderson B and Martindale W. Towards optimizing rowing technique. Medicine and Science in Sports and Exercise 1986; 18: 454-468.

12. Serveto S, Barre S, Kobus JM and Mariot JP. A three-dimensional model of the boat-oars-rower system using ADAMS and LifeMOD commercial software. Proceedings of the Institution of Mechanical Engineers, Part P: Journal of Sports Engineering and Technology 2010; 224: 75-88.

13. Zatsiorsky VM and Yakunin N. Mechanics and biomechanics of rowing: A review. International Journal of Sport Biomechanics 1991; 7: 229-281.

14. Adam K, Lenk H, Nowacki P, Rulffs M and Schröder W. Rudertraining. [Rowing training.] Germany: Limpert, 1977.

15. Nolte V. Shorter Oars Are More Effective. Journal of Applied Biomechanics 2009; 25: 1-8.

16. Hofmijster M, De Koning J and Van Soest AJ. Estimation of the energy loss at the blades in rowing: Common assumptions revisited. Journal of Sports Science 2010; 28: 1093-1102.

17. Sliasas $A$ and Tullis $S$. Modelling the effect of oar shaft bending during the rowing stroke. Proceedings of the Institution of Mechanical Engineers Part P Journal of Sports Engineering and Technology 2011; 225: 265-270. 


\section{Chapter 2}

\section{The Mechanical Properties of Oar-Shafts}

\subsection{Introduction}

Rowing oar-shafts transfer force applied to the handles by the rower to the blades, which act on the water to propel the boat. The oar-shaft's efficiency [1] in transferring force is affected by its mechanical design and material composition. Until the 1980s, oar-shafts were predominantly made out of Sitka spruce from the northern United States and Canada because their shorter growing seasons produced a finer grain [2, 3]. The wood was cut into strips, laminated, shelved for weeks, and finished by hand cutting and polishing [3-5]. Ash was occasionally glued onto the oar-shafts for added durability [4]. The Sitka spruce oar-shafts tapered from the sleeves towards the blades [5], and had masses of 4 to $4.3 \mathrm{~kg}$ [3].

Most present-day oar-shafts are engineered from composite materials, like carbon fiber reinforced polymers that are cured at high temperatures [2-4, 6]. These composite oar-shafts are stiffer, and up to $60 \%$ lighter, than wood oarshafts [4-6]. Oar manufacturers typically classify a shaft's stiffness based on its deflection at the junction between the shaft and the blade, when a static load of 98.1 $\mathrm{N}$ is applied to the junction area [7-9]. Its stiffness can be affected by the amount and distribution of high-modulus carbon fiber. For instance, Concept2 designs and markets "Extra-Soft" shafts, which contain approximately $20 \%$ high modulus carbon fiber, and "Medium" stiffness oar-shafts with about $40 \%$; Medium oar-shafts are heavily loaded with high modulus carbon fiber near the sleeves (D. Dreissigacker. 2013, personal communication). Medium oar-shafts are the most popular among competitive rowers [2]. However, oars are typically selected based on the rowers' subjective preferences [3] rather than quantitative analysis. 
The mechanical properties of rowing oar-shafts are not well known. Many previous studies have assumed that the oar-shaft is perfectly rigid [10-20]. This assumption is simple but unrealistic, since oar-shaft deflections can be seen with the naked eye during on-water rowing (Figure 4). Sliasas and Tullis [21] estimated a maximum deflection of $0.0164 \mathrm{~m}$ in $3.75 \mathrm{~m}$ sweep oars by comparing the angular velocities measured at the oarlocks with those calculated at the blades through beam theory. Hofmijster et al. [22] looked at the effect oar-shaft deflections have on rowing biomechanics. Instrumented oars and oarlocks were dynamically loaded with up to $150 \mathrm{~N}$ using an end-loaded cantilever beam system, and relations were calculated between the measured signals, oar-shaft deflection and blade force. A "world class" rower was subsequently tested at race pace over $500 \mathrm{~m}$ using the instrumented equipment. The blade kinematics during the drive were reconstructed from the on-water measurements and compared with those of a perfectly rigid oar [22]. Compared to the tested oars, the rigid-oar assumption changed the reconstructed blade kinematics during the drive, which changed the hydrodynamic forces calculated on the blades [22]. 


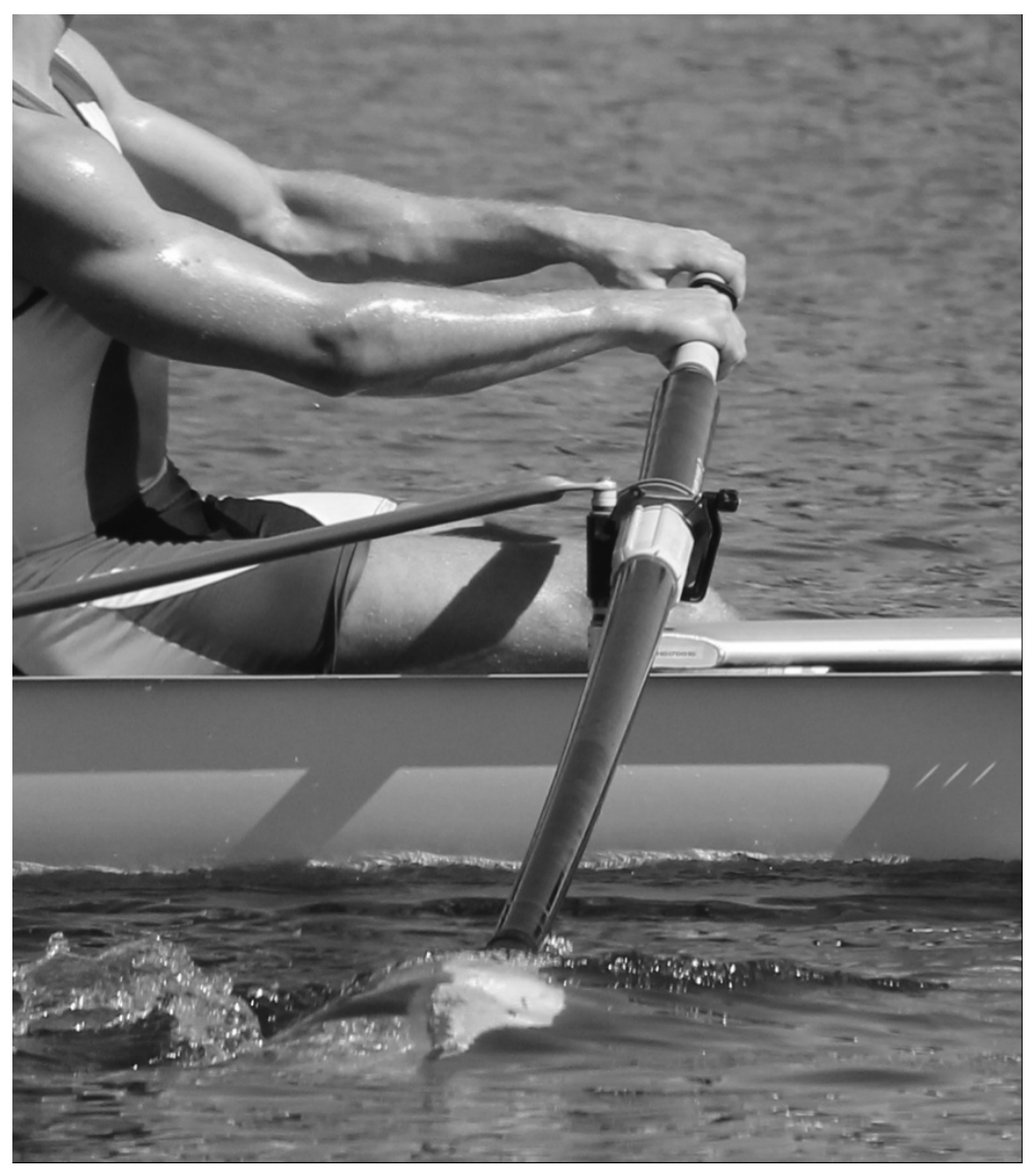

Figure 4. Photograph of a sweep oar-shaft during on-water rowing with visible deflection.

The angle of attack AOA is the angle between the blade's reference line and the vector representing the oncoming flow of water. The reference line is an imaginary straight line joining the leading and trailing edges of the blade. The AOA has implications for the hydrodynamic forces on the blades [7, 23-24]. As the blade moves through the water and the oar-shaft deflects, the AOA may differ from that of a perfectly rigid oar-shaft, resulting in different hydrodynamic forces on the blades. An oar-shaft's stiffness and length can affect its deflection during on-water rowing, and thus has implications for boat propulsion. In this chapter, the deflection of oar-shafts was experimentally studied as a function of length and stiffness, and compared to the behaviour of a homogenous end-loaded cantilever beam. 


\subsection{Methods}

Two sets of sculling oars with "skinny" shafts (Concept2 Inc., Vermont, United States) of different stiffness were investigated. Medium oar-shafts, which are designed to deflect $0.045 \pm 0.002 \mathrm{~m}$ at the junction between the shaft and the blade when loaded with $98.1 \mathrm{~N}$, are referred to as "M" oars; oar-shafts denoted as "ES" are Extra-Soft and designed to deflect $0.065 \pm 0.002 \mathrm{~m}$ [8]. The circumferences of Oar M and Oar ES both taper from $0.111 \mathrm{~m}$ at the sleeves to $0.108 \mathrm{~m}$ at the blades. Oar M and Oar ES have masses of 1.4 and $1.3 \mathrm{~kg}$, respectively.
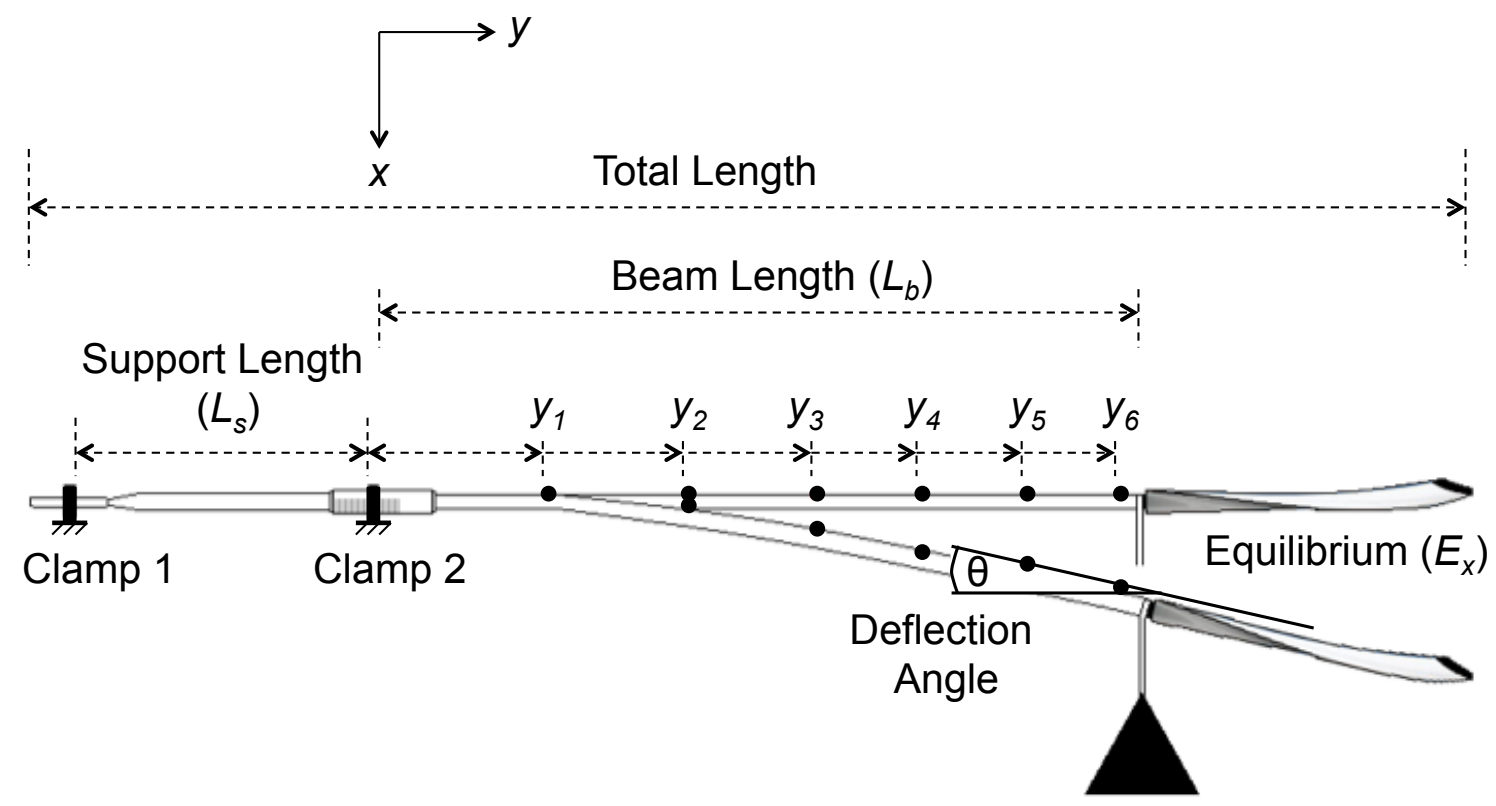

Static Load $(\boldsymbol{W})$

Figure 5. Schematic of the setup used to measure the oar-shaft's deflection from its equilibrium position $E_{x}$ at six positions $y_{i}$ along the shaft when a static load $\boldsymbol{W}$ is applied at length $L_{b}$ from Clamp 2. The support length $L_{s}$ is the distance between Clamps 1 and 2 . The deflection angle at the blade-end of the oar-shaft is denoted by $\theta$.

The experimental set-up is illustrated schematically in Figure 5 and a photograph is shown in Figure 6. The oars were clamped at the handle (1) and sleeve (2), simulating the rower's hand and oarlock, respectively. The distance 
between Clamps 1 and 2 (i.e., the support length $L_{s}$ ) was fixed at $0.715 \mathrm{~m}$. The beam length $L_{b}$ is the distance between the load and Clamp 2, and ranged between 1.279 and $1.319 \mathrm{~m}$. The total length of the oars, which were set to 2.66 , 2.68 and $2.70 \mathrm{~m}$, varied by changing $L_{b}$. All length measurements were taken with $a \pm 9 \times 10^{-5} \mathrm{~m}$ tolerance (Lufkin, Texas, USA). The oars have a built-in length adjustment system, whereby the oar can adjust up to $0.05 \mathrm{~m}$ in total length [8]. Six positions were marked along the oar-shafts at $y_{1-6}$ measured from Clamp 2 (i.e., where $y$ is zero). A digital height gage (Mitutoyo Inc., Quebec, Canada), with $a \pm 5 \times 10^{-5} \mathrm{~m}$ tolerance, was used to measure the linear deflection $\delta$ in the $x$-axis at positions $y_{1-6}$ relative to the oar-shafts equilibrium position $E_{x}$. Port (P) and starboard (S) versions of Oar M and Oar ES were tested with three different lengths, for a total of twelve configurations (Table 1).

Table 1. The twelve oar configurations that were investigated. Each configuration is designated by a code that indicates the stiffness (M or ES), side (P or S), and total length of the oar. The total length of the oar varied by changing $L_{b}$.

\begin{tabular}{ccccc}
\hline Code & Stiffness & Side & Total Length $(\mathrm{m})$ & $L_{b}(\mathrm{~m})$ \\
\hline MP2.66 & Medium & Port & 2.66 & 1.279 \\
MP2.68 & Medium & Port & 2.68 & 1.299 \\
MP2.70 & Medium & Port & 2.70 & 1.319 \\
MS2.66 & Medium & Starboard & 2.66 & 1.279 \\
MS2.68 & Medium & Starboard & 2.68 & 1.299 \\
MS2.70 & Medium & Starboard & 2.70 & 1.319 \\
ESP2.66 & Extra-Soft & Port & 2.66 & 1.279 \\
ESP2.68 & Extra-Soft & Port & 2.68 & 1.299 \\
ESP2.70 & Extra-Soft & Port & 2.70 & 1.319 \\
ESS2.66 & Extra-Soft & Starboard & 2.66 & 1.279 \\
ESS2.68 & Extra-Soft & Starboard & 2.68 & 1.299 \\
ESS2.70 & Extra-Soft & Starboard & 2.70 & 1.319 \\
\hline
\end{tabular}


Static loads $\boldsymbol{W}$ of $12.75,21.57,41.19,111.8,152$, and $201.04 \mathrm{~N}$ were individually applied to the oars at distances $L_{b}$ from Clamp 2 by suspending weight plates from the shafts using a tether. The weights of the plates and tether were measured using a digital bench scale (Rice Lake Weighing Systems, Wisconsin, United States) with an engineering tolerance of $\pm 0.98 \mathrm{~N}$. The range of $\boldsymbol{W}$ was chosen to parallel the range of forces previously estimated on the blade during on-water rowing [22]. Four measurement trials were conducted for each value of $\boldsymbol{W}$ and $y$, and the results are arithmetic means with the uncertainties given by standard deviations SD. Error bars are not included in the figures because they were smaller than the symbols. This demonstrates that any measurement errors are substantially smaller than the measured deflections. All figures were generated in MATLAB 2013a (The MathWorks Inc., Massachusetts, USA).

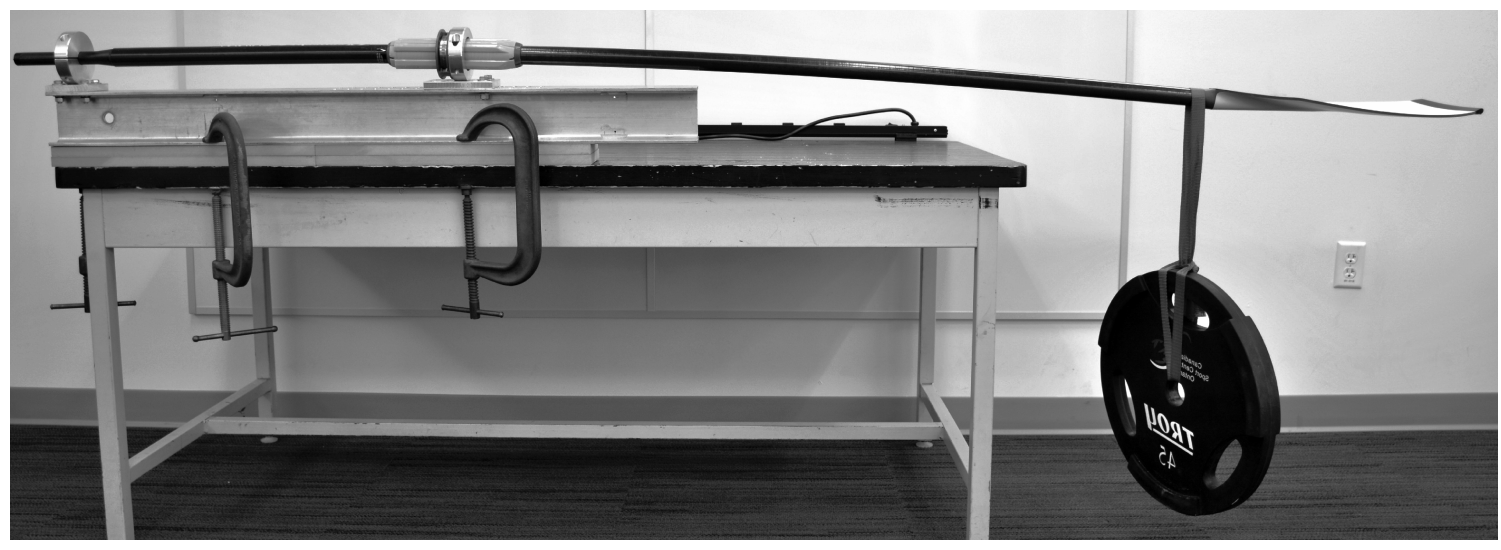

Figure 6. Photograph of the experimental set-up used to measure oar-shaft deflection. The oar is secured to a laboratory bench with a custom-made support stand, and a weight plate is suspended from the oar-shaft using a tether.

\subsection{Results}

Figure 7 shows $\delta$ as a function of $y$ for MP2.70 and ESP2.70 when loaded with $111.8 \mathrm{~N}$. As expected, Oar ES deflected more than Oar $M$ at $y_{1-6}$, with a maximum difference in deflection of $29.5 \%$. The differences in deflection 
between the two oars increased with distance towards the blades. This trend was seen in all the configurations over the range of $\boldsymbol{W}$ studied. Deflection data were fit to the expression for the deflection of an end-loaded cantilever beam with a homogenous cross-section [25] given by

$\boldsymbol{\delta}_{y_{i}}=\frac{W y_{i}^{2}}{6 E I}\left(3 L_{b}-y_{i}\right)$

where $E$ is the Young's modulus, $I$ is the area moment of inertia, and the combination $E l$ is the flexural rigidity. The weight of the beam is neglected in this model. Since the values of $\boldsymbol{W}$ and $L_{b}$ are known, $E /$ was the only fitting parameter. Typical fits are shown in Figure 7. While equation (1) models the deflection of the oar-shafts qualitatively, it systematically under predicts the deflection at small $y_{i}$ and over predicts the deflection at large $y_{i}$. Note that the model is based on an end-loaded cantilever beam while the experimental setup arguably resembles a three-point bending flexural test. 


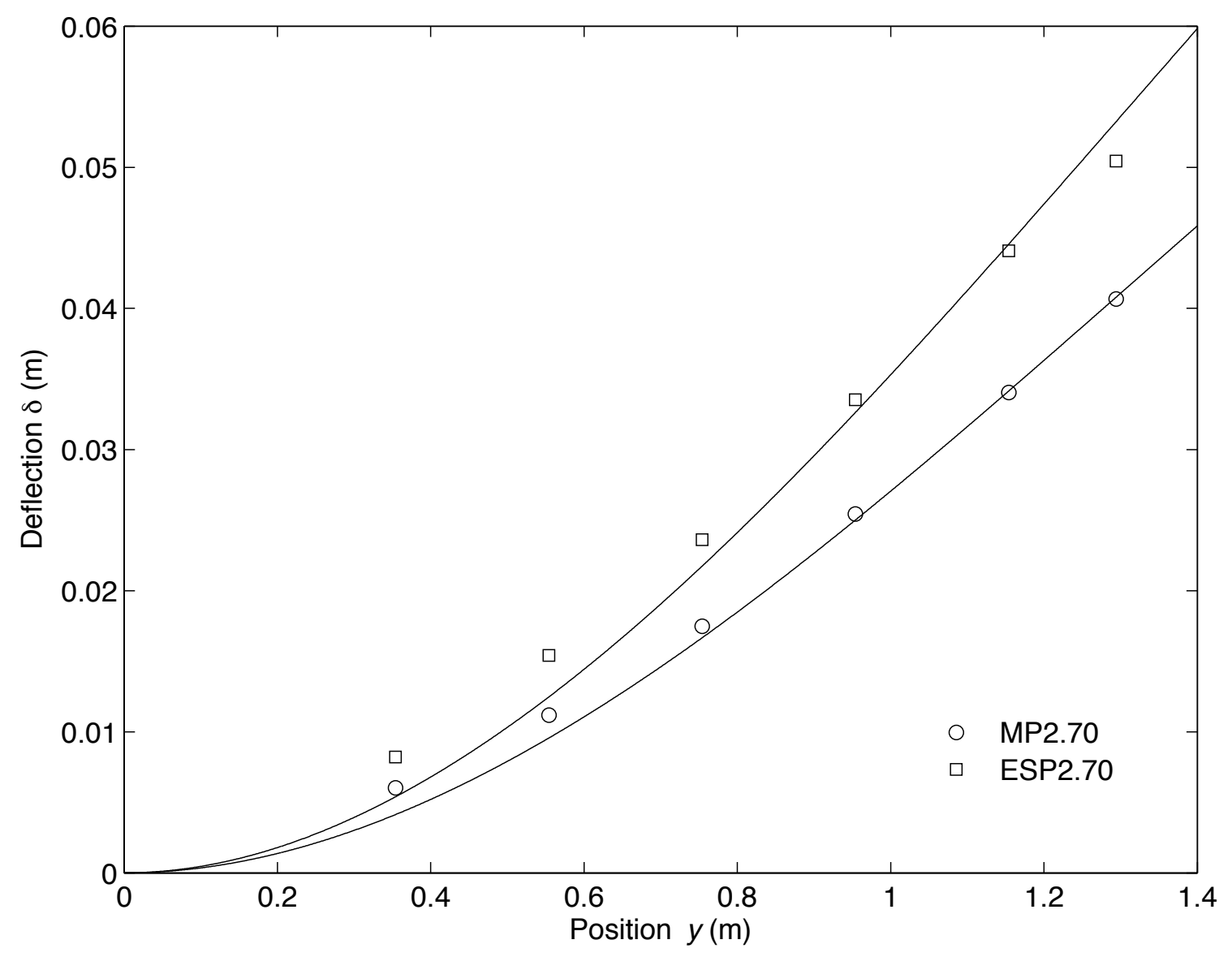

Figure 7. Deflection $\delta$ as a function of position $y$ for MP2.70 and ESP2.70 when loaded with $111.8 \mathrm{~N}$. The curves are fits to equation (1). 


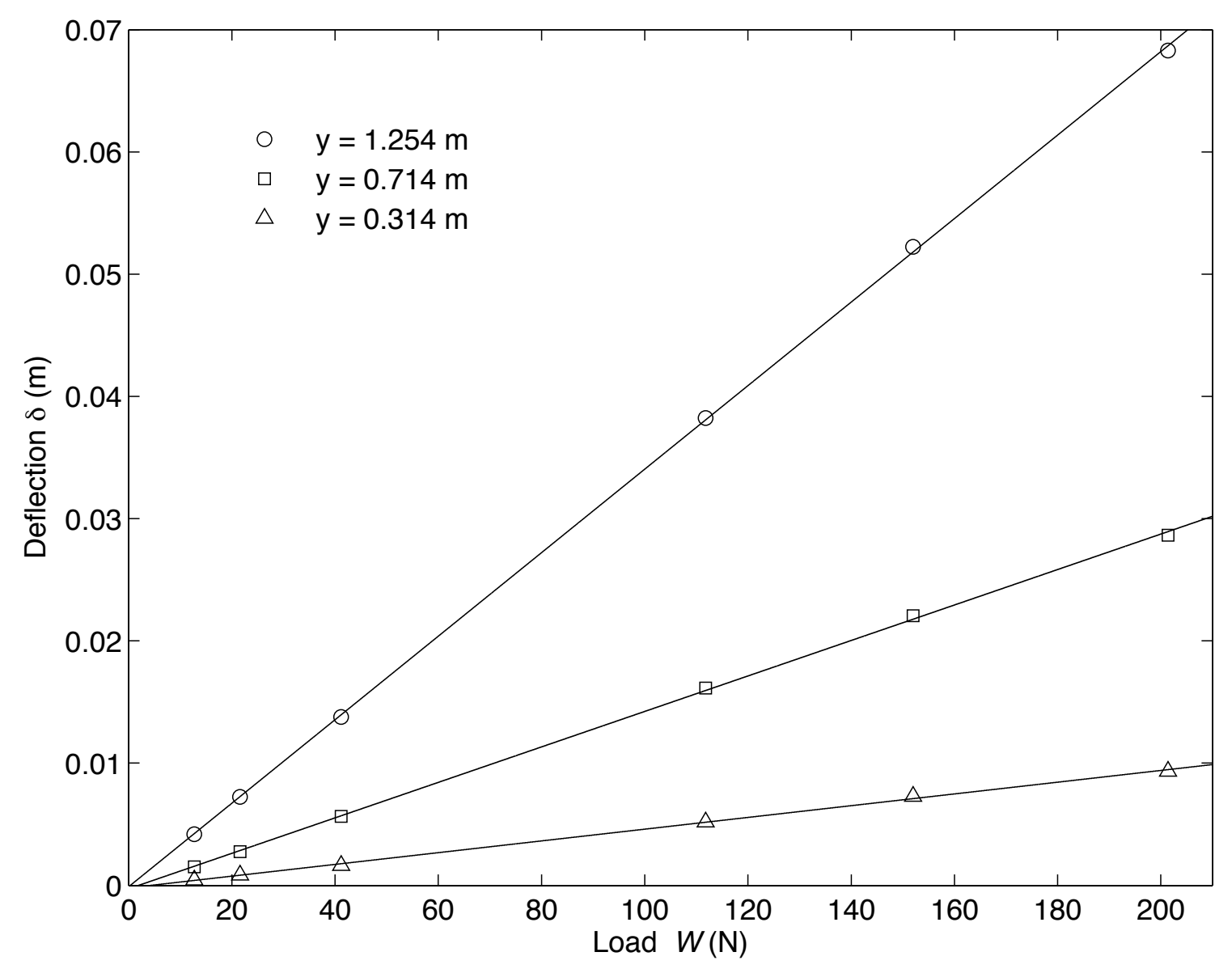

Figure 8. Deflection $\delta$ as a function of load $\boldsymbol{W}$ for MP2.66 at three positions $y_{i}$ along the oar-shaft. The lines are fits to the data discussed in the text.

Figure 8 shows $\delta$ as a function of $\boldsymbol{W}$ for MP2.66 at three positions $y$. At each $y_{i}, \boldsymbol{\delta}$ was accurately proportional to $\boldsymbol{W}$, as expected from Hooke's Law; this trend was seen in all the configurations. The slope $m$ of the data for a given $y_{i}$ is the compliance of the oar-shaft at that position, and is related to the flexural rigidity El via

$E I_{y_{i}}=\frac{y_{i}{ }^{2}}{6 m_{y_{i}}}\left(3 L_{b}-y_{i}\right)$

Typical results for $E I$ as a function of $y$ are illustrated for two oar configurations in Figure 9. Both oars are most compliant near the sleeves and become progressively stiffer towards the blades. Similar results were seen in all 
the configurations whereby the blade-end of the oar-shafts are stiffer, by up to $64.4 \%$ for Oar $\mathrm{M}$ and $78.9 \%$ for Oar ES, than compared to near the sleeves. In Figure $9, E I$ is approximately $33.9 \%$ larger for Oar M than Oar ES.

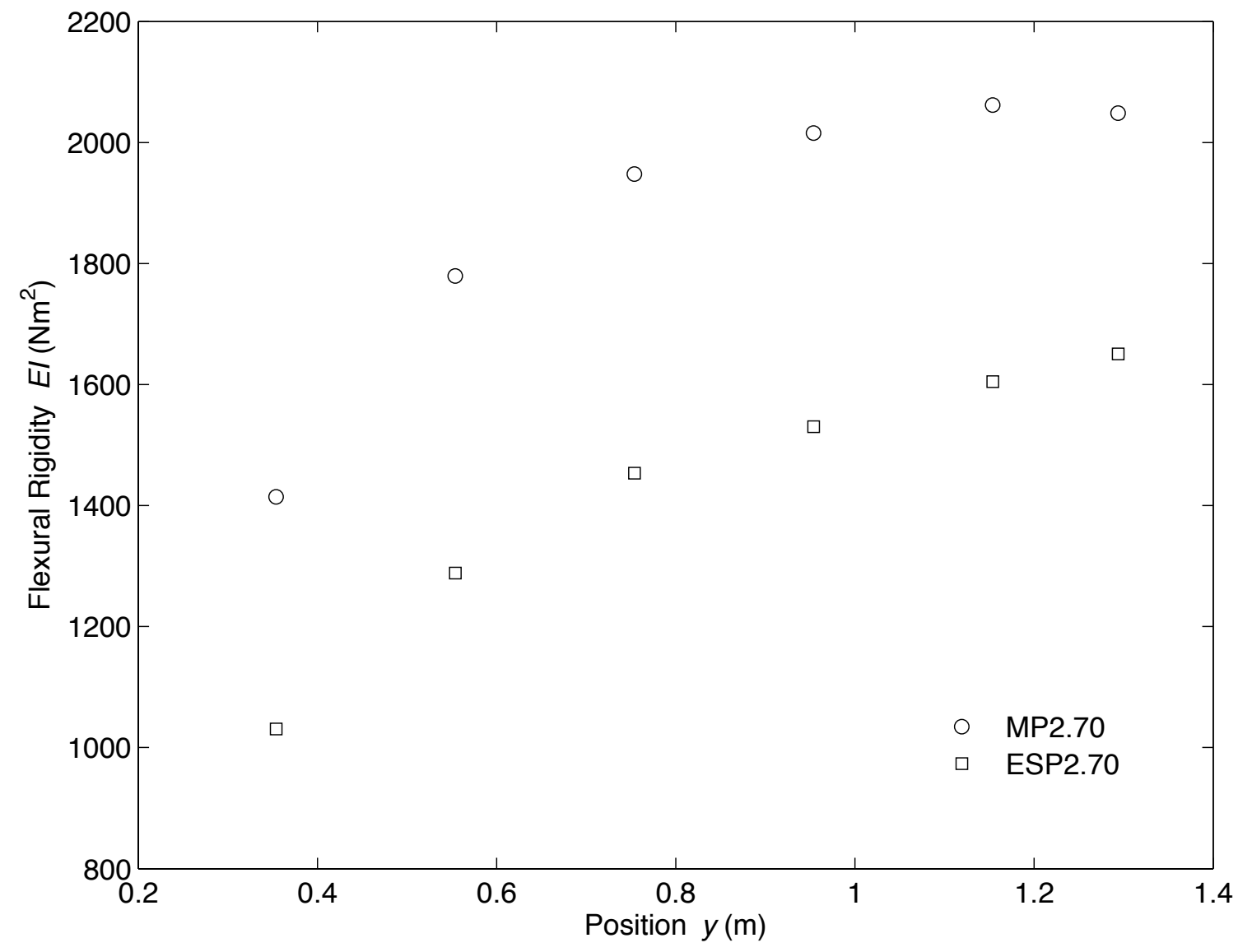

Figure 9. Flexural rigidity $\mathrm{El}$ as a function of position $y$ for MP2.70 and ESP2.70.

Figure 10 shows $E l$ as a function of $y$ for port and starboard versions of Oar $\mathrm{M}$ at three different lengths. For a given $y_{i}$, increasing the oar length from 2.66 to $2.70 \mathrm{~m}$ increased $E /$ by up to $17.5 \%$. Likewise, there was a $13.7 \%$ maximum difference in $E l$ between port and starboard oars of the same length. The differences between port and starboard oars presumably reflect uncontrolled variations in the manufacturing process, and are substantially smaller than the differences in $E l$ between oar-shafts of different stiffness. Table 2 shows the $E I$ values for all twelve configurations. 


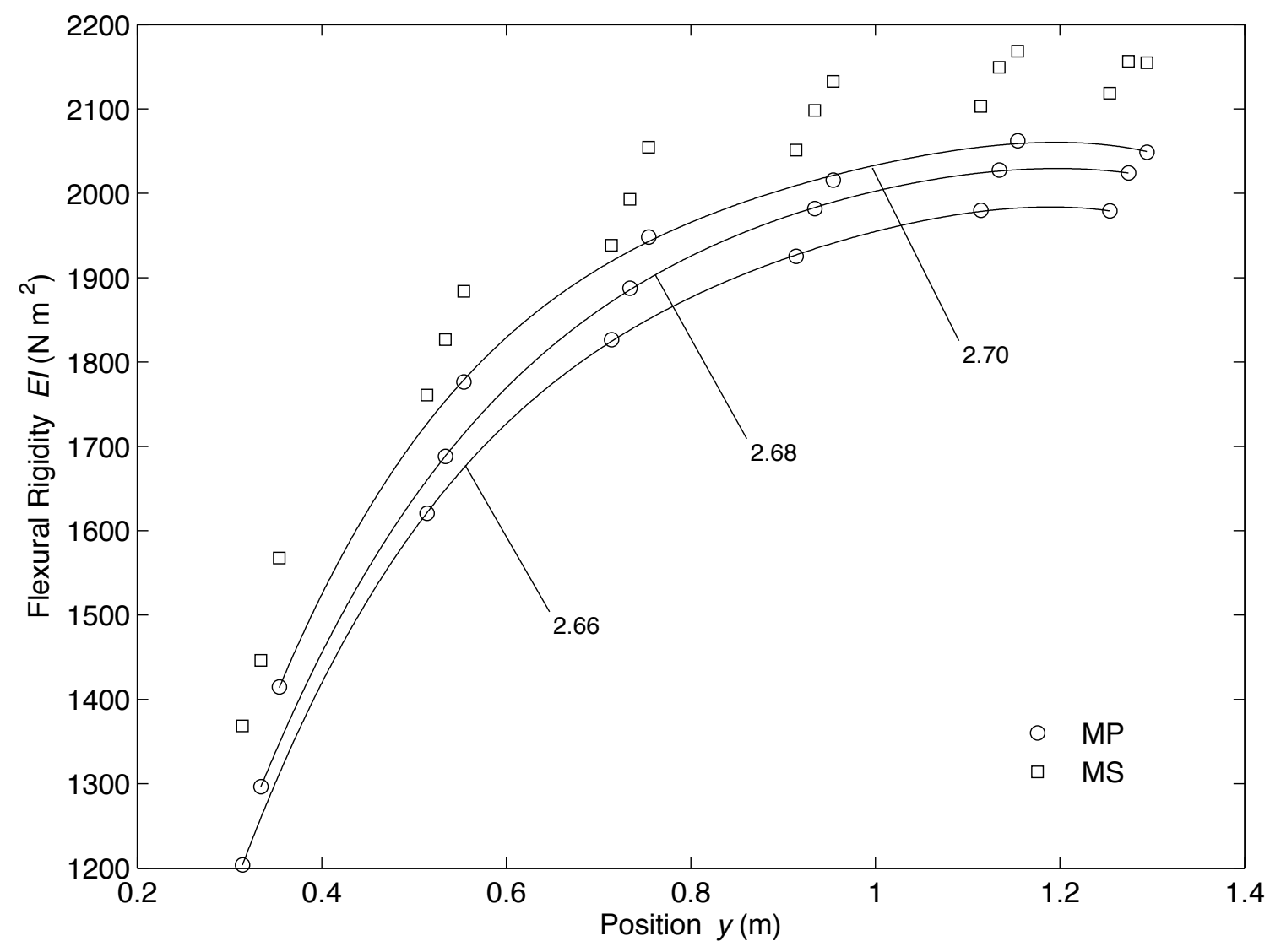

Figure 10. Flexural rigidity $E l$ as a function of position $y$ for port and starboard versions of Oar M. The three data points in each group correspond to oar lengths of $2.66,2.68$, and $2.70 \mathrm{~m}$, as shown by the sketched curves. 
Table 2. The flexural rigidity $E I\left(\mathrm{Nm}^{2}\right)$ for each oar configuration at each position $y_{i}$ along the oar-shaft. The uncertainties are SD.

\begin{tabular}{ccccccc}
\hline Code & $y_{1}$ & $y_{2}$ & $y_{3}$ & $y_{4}$ & $y_{5}$ & $y_{6}$ \\
\hline MP2.66 & $1246.8 \pm 3.2$ & $1646.4 \pm 2.6$ & $1863.1 \pm 1.7$ & $1946.2 \pm 1.1$ & $1998.0 \pm 0.6$ & $1992.7 \pm 1.0$ \\
MP2.68 & $1321.6 \pm 2.9$ & $1724.5 \pm 6.3$ & $1926.2 \pm 1.3$ & $2009.1 \pm 2.2$ & $2047.7 \pm 1.3$ & $2037.7 \pm 1.2$ \\
MP2.70 & $1439.5 \pm 9.7$ & $1784.1 \pm 5.2$ & $1953.7 \pm 4.5$ & $2027.9 \pm 2.9$ & $2074.6 \pm 3.8$ & $2062.9 \pm 5.4$ \\
MS2.66 & $1370.9 \pm 4.4$ & $1768.3 \pm 4.9$ & $1954.8 \pm 5.0$ & $2060.7 \pm 3.4$ & $2112.7 \pm 1.7$ & $2130.5 \pm 1.6$ \\
MS2.68 & $1441.4 \pm 3.6$ & $1828.3 \pm 3.2$ & $2007.5 \pm 2.3$ & $2106.2 \pm 4.8$ & $2157.9 \pm 1.2$ & $2164.9 \pm 2.7$ \\
MS2.70 & $1533.2 \pm 9.9$ & $1879.7 \pm 8.1$ & $2055.2 \pm 3.6$ & $2138.5 \pm 3.1$ & $2166.6 \pm 1.7$ & $2169.0 \pm 3.5$ \\
ESP2.66 & $919.9 \pm 2.5$ & $1227.7 \pm 4.6$ & $1400.3 \pm 1.7$ & $1509.3 \pm 1.0$ & $1544.2 \pm 1.0$ & $1611.5 \pm 0.8$ \\
ESP2.68 & $980.5 \pm 2.6$ & $1255.0 \pm 2.9$ & $1424.6 \pm 2.4$ & $1534.5 \pm 0.9$ & $1583.1 \pm 1.8$ & $1624.8 \pm 1.7$ \\
ESP2.70 & $1033.1 \pm 6.6$ & $1292.4 \pm 3.1$ & $1455.8 \pm 0.8$ & $1537.3 \pm 1.2$ & $1603.2 \pm 2.0$ & $1658.4 \pm 0.7$ \\
ESS2.66 & $939.7 \pm 2.4$ & $1230.2 \pm 2.7$ & $1392.8 \pm 2.3$ & $1499.5 \pm 1.4$ & $1561.4 \pm 1.1$ & $1579.0 \pm 0.6$ \\
ESS2.68 & $976.2 \pm 2.7$ & $1234.4 \pm 3.6$ & $1387.7 \pm 2.0$ & $1492.7 \pm 1.5$ & $1564.1 \pm 1.4$ & $1584.1 \pm 0.9$ \\
ESS2.70 & $992.7 \pm 4.5$ & $1244.6 \pm 3.4$ & $1408.0 \pm 1.3$ & $1504.8 \pm 1.0$ & $1570.9 \pm 0.2$ & $1600.7 \pm 0.6$ \\
\hline
\end{tabular}

The deflection angle $\theta$ was calculated from the tangent between the deflections at the two positions $y_{5}$ and $y_{6}$ at the blade-end of the oar-shafts, as illustrated in Figure 5. Figure 11 shows $\theta$ as a function of $\boldsymbol{W}$ for MP2.70 and ESP2.70. As expected, $\theta$ increased proportionally with $\boldsymbol{W}$. The relative differences in $\theta$ between the two oars also increased with $\boldsymbol{W}$, with $\theta$ being $1.18 \pm$ $0.01^{\circ}$ larger for ESP2.70 than for MP2.70 when $\boldsymbol{W}=201.04 \mathrm{~N}$. The angle of deflection at the free end of a homogenous end-loaded cantilever beam [25] is given by

$\theta_{\text {beam }}=\frac{W L_{b}{ }^{2}}{2 E I_{y_{6}}}$

The linear fits in Figure 11 are based on the predications from equation (3) using the $E I$ values at $y_{6}$ calculated from equation (2). The model provides a good description of the oar-shafts' behaviour. 


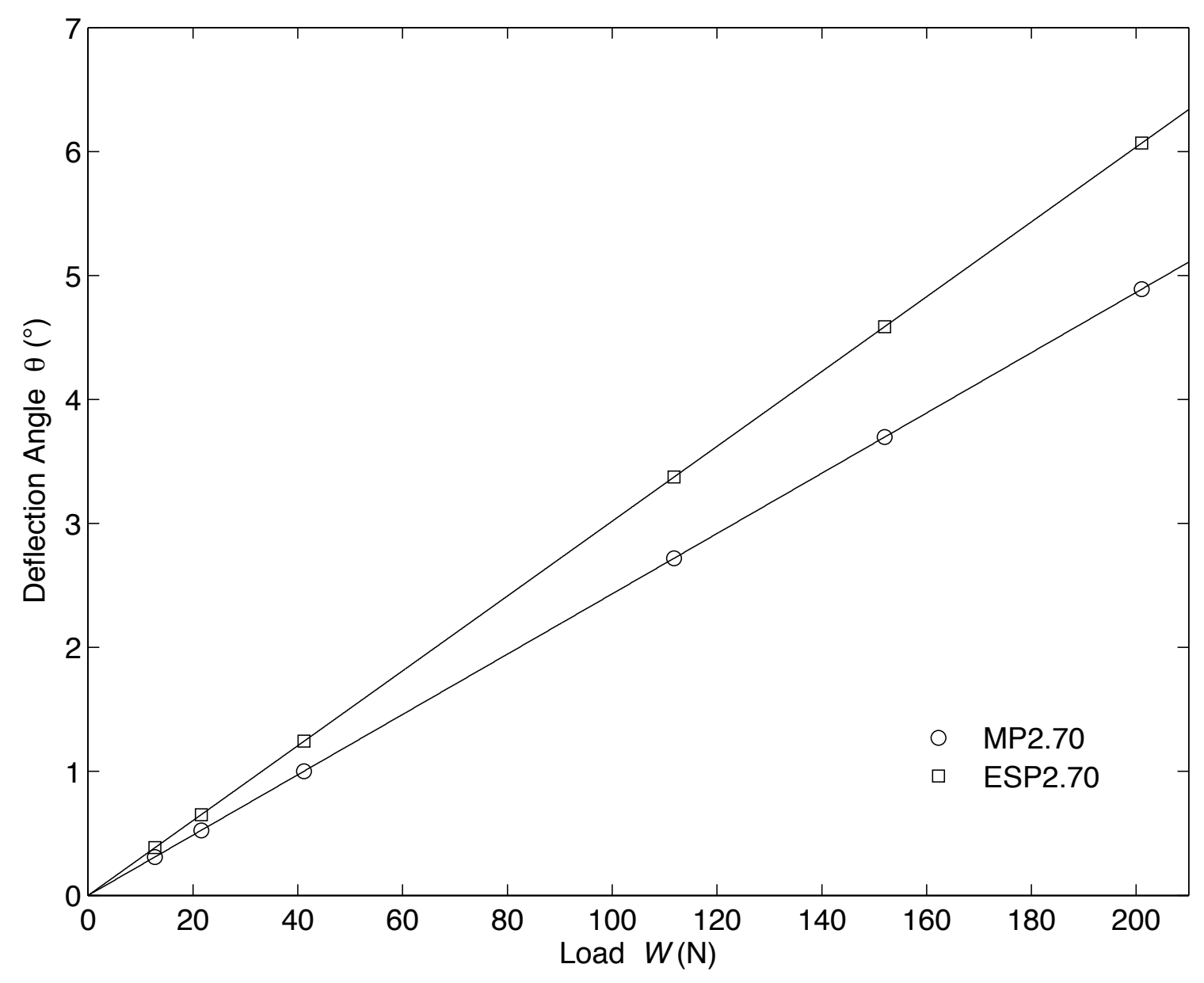

Figure 11. Deflection angles $\theta$ at the blade-end of the oar-shafts for MP2.70 and ESP2.70 as a function of load $\boldsymbol{W}$. The linear fits are expressions to equation (3).

Increasing oar length from 2.66 to $2.70 \mathrm{~m}$ increased $\theta$ by up to $0.20 \pm$ $0.07^{\circ}$ when loaded with $201.04 \mathrm{~N}$. Similarly, there was a maximum difference in $\theta$ of $0.22 \pm 0.07^{\circ}$ between port and starboard oars of the same stiffness and length. Table 3 shows the calculated values of $\theta$ for all twelve configurations. 
Table 3. The deflection angles $\theta$ at the blade-end of the oar-shafts for each configuration when loaded with $201.04 \mathrm{~N}$. The uncertainties are SD.

\begin{tabular}{cc}
\hline Code & Deflection Angle $\left(^{\circ}\right)$ \\
\hline MP2.66 & $4.73 \pm 0.002$ \\
MP2.68 & $4.77 \pm 0.003$ \\
MP2.70 & $4.86 \pm 0.013$ \\
MS2.66 & $4.42 \pm 0.003$ \\
MS2.68 & $4.49 \pm 0.006$ \\
MS2.70 & $4.62 \pm 0.007$ \\
ESP2.66 & $5.85 \pm 0.003$ \\
ESP2 2.68 & $5.98 \pm 0.006$ \\
ESP2.70 & $6.04 \pm 0.002$ \\
ESS2.66 & $5.97 \pm 0.002$ \\
ESS2.68 & $6.13 \pm 0.003$ \\
ESS2.70 & $6.26 \pm 0.003$ \\
\hline
\end{tabular}

\subsection{Discussion}

The aim of this chapter was to investigate the mechanical properties of oar-shafts with different stiffness and length. Oars were clamped at the handles and sleeves, and statically loaded at the blade-end of the oar-shafts; deflections were measured at several positions along the shafts. This technique provides more information than previous investigations [7-9], which measured deflection simply at the junction between the shaft and the blade. The use of static loads to test the mechanical properties of the oar-shafts is consistent with the methods used by oar manufacturers to design and determine oar-shaft stiffness [8].

As expected, Extra-Soft oar-shafts deflected more than Medium oarshafts. Concept2 specifies that Extra-Soft and Medium oar-shafts deflect 
approximately 0.065 and $0.045 \mathrm{~m}$ when a static load of $98.1 \mathrm{~N}$ is applied at a distance of $1.50 \mathrm{~m}$ from the support [8]. To compare with the manufacturers specifications, the fits to deflection as a function of load at a beam length of $1.319 \mathrm{~m}$ were used to estimate the deflection at $98.1 \mathrm{~N}$. The calculated deflections for the Extra-Soft and Medium oar-shafts were approximately 30 and $22 \%$ lower than the specified deflections. Since the oar-shafts are most compliant near the sleeves and Concept 2 uses a $12 \%$ longer distance between the support and load, this could explain why Concept2 measures larger deflections.

There were slight differences in the deflections between starboard and port oars of the same stiffness and length. This is likely not intentional, but rather a result of variations in the manufacturing process. It is expected that similar variations would be seen between, for example, several port oars. Asymmetric transfer of force from the handles to the port and starboard blades could produce a moment of force about the z-axis [10]. This would lead to what is known as yaw rotation. Yaw rotation has a negative effect on boat velocity, since altering the boat's main direction of motion increases form drag on the underside of the shell [26-29]. However, differences in force between port and starboard oars are assumed to be predominately caused by the rower's asymmetric application of force to the handles as oppose to any mechanical differences between the port and starboard oars, like the ones measured in this work.

The deflection angle at the blade-end of the oar-shaft was affected by both the oar-shaft's stiffness and length. However, the effect of stiffness on the deflection angle was large compared to the effect of increasing oar length from 2.66 to $2.70 \mathrm{~m}$. Differences in deflection angle have implications for on-water rowing because they may affect the AOA for a given oar angle. The AOA has implications for the hydrodynamic forces on the blades [7, 23-24]. There was a maximum difference in the deflection angle of $1.18 \pm 0.01^{\circ}$ between the ExtraSoft and Medium oar-shafts when loaded with 201.04 N. In contrast, the angular displacement of the oar-blade about the pin throughout the drive for an elite 
sculling rower, averaged over 20 strokes at a rate of 20 strokes $/ \mathrm{min}$, is reported to be $109.1 \pm 1.8^{\circ}$ (Canadian Sport Institute Ontario, 2014, personal communication). The change in deflection angle due to changing oar-shaft stiffness was thus smaller than the inter-stroke inconsistencies in the oar angles (i.e., the uncertainties) for an elite sculling rower.

The results show that the flexural rigidity of Concept2 oars is not constant along the shafts. For instance, differences of up to $775.30 \mathrm{Nm}^{2}$ were calculated between the sleeve and the blade for MP2.66. The oar-shafts are most compliant near the sleeves and become progressively stiffer towards the blades. These findings can enhance the accuracy of numerical models that rely on the properties of the oar-shafts for input parameters because they provide more information than compared to previous research [21], which assumed a constant flexural rigidity of $8668 \mathrm{Nm}^{2}$ along the Concept2 oar-shaft. In addition, since the location of the point of force application on the blade seemingly varies with respect to drive time [30], investigating the oar-shaft's torsional rigidity presents an interesting topic future research.

In summary, the mechanical properties of oar-shafts with different stiffness and length were investigated, and their implications for boat propulsion and rowing performance discussed. Actual rowing performance, however, depends on many factors unrelated to the properties of the equipment, such as the technique of the rower and the weather conditions. Incorporating these factors would contribute to a more realistic analysis of the effects of oar-shaft mechanics on rowing performance.

\subsection{References}

1. Cabrera DS and Ruina AL. Propulsive Efficiency of Rowing Oars. Report, Cornell University, USA, February 2006.

2. McNeely E and Royle M. Skillful Rowing. UK: Meyer \& Meyer Sport, 2002. 
3. Ashby MF. Materials selection in mechanical design. 3rd ed. UK: Elsevier Butterworth-Heinemann, 2005.

4. Boyne DJ. Essential Sculling: An Introduction to Basic Strokes, Equipment, Boat Handling, Technique, and Power. Canada: Globe Pequot Press, 2000.

5. Ritchie AC. Effect of Oar Design on the Efficiency of the Rowing Stroke. In: Subic A, Fuss FK and Ujihashi S (eds) The Impact of Technology on Sport II. UK: Taylor \& Francis Group, 2007, pp.509-512.

6. Maybery K. Rowing: The essential guide to equipment and technique. UK: New Holland Publishers, 2002.

7. Macrossan MN. The direction of the water force on a rowing blade and its effect on efficiency. Report, University of Queensland, Australia, March 2008.

8. Concept2. Oar-Shafts Stiffness Options, http://www.concept2.com/oars/oaroptions/shafts/stiffness (accessed 9 April 2014).

9. Brača-sport Rowing. Oar-Shaft Stiffness, http://rowing.bracasport.com/oars/shafts/shaft-stiffness.html (accessed 9 April 2014).

10. Baudouin A and Hawkins D. A biomechanical review of factors affecting rowing performance. British Journal of Sports Medicine 2002; 36: 396-402.

11. Brearley MN and De Mestre NJ. Modeling the rowing stroke and increasing its efficiency. In: The third Conference on Mathematics and Computers in Sport, Bond University, Queensland, Australia, 30 Sept-2 Oct 1996, pp.35-46.

12. Cabrera D, Ruina A and Kleshnev V. A Simple 1+ Dimensional Model of Rowing Mimics Observed Forces and Motions. Human Movement Science 2006; 25: 192-220. 
13. Hofmijster MJ, Landman EH, Smith RM and Van Soest AJ. Effect of stroke rate on the distribution of net mechanical power in rowing. Journal of Sports Sciences 2007; 25: 403-411.

14. Sanderson B and Martindale W. Towards optimizing rowing technique. Medicine and Science in Sports and Exercise 1986; 18: 454-468.

15. Zatsiorsky VM and Yakunin N. Mechanics and biomechanics of rowing: A review. International Journal of Sport Biomechanics 1991; 7: 229-281.

16. Findlay M and Turnock SR. Mechanics of a rowing stroke: Surge speed variations of a single scull. Proceedings of the Institution of Mechanical Engineers, Part P: Journal of Sports Engineering and Technology 2010; 224: 89100.

17. Affeld K, Schichl K and Ziemann A. Assessment of rowing efficiency. International Journal of Sports Medicine 1993; 14: 39-41.

18. Serveto S, Barre S, Kobus JM and Mariot JP. A three-dimensional model of the boat-oars-rower system using ADAMS and LifeMOD commercial software. Proceedings of the Institution of Mechanical Engineers, Part P: Journal of Sports Engineering and Technology 2010; 224: 75-88.

19. Macrossan MN and Macrossan NW. Energy efficiency of the rowing oar from catch to square-off. Report, University of Queensland, Australia, May 2008.

20. Alexander FH. The theory of rowing. Proceedings of the University of Durham Philosophical Society 1925, pp.160-179.

21. Sliasas $A$ and Tullis $S$. Modelling the effect of oar shaft bending during the rowing stroke. Proceedings of the Institution of Mechanical Engineers Part P Journal of Sports Engineering and Technology 2011; 225: 265-270. 
22. Hofmijster M, De Koning J and Van Soest AJ. Estimation of the energy loss at the blades in rowing: Common assumptions revisited. Journal of Sports Science 2010; 28: 1093-1102.

23. Coppel A, Gardner T, Caplan N and Hargreaves D. Simulating the fluid dynamic behaviour of oar blades in competition rowing. Proceedings of the Institution of Mechanical Engineers Part P - Journal of Sports Engineering and Technology 2010; 224: 25-35.

24. Nolte V. Shorter Oars Are More Effective. Journal of Applied Biomechanics 2009; 25: 1-8.

25. Gere JM. Mechanics of Materials. 5th ed. USA: Brooks/Cole Publishing Company, 2002.

26. Schneider $E$ and Hauser M. Biomechanical analysis of performance in rowing. In: Morecki A, Fidelus K, Kedzior K and Wit A (eds) Biomechanics VII-B. USA: University Park Press, 1981, pp.430-435.

27. Smith RM and Spinks WL. Discriminant analysis of biomechanical differences between novice, good and elite rowers. Journal of Sports Science 1995; 13: 377385.

28. Loschner C, Smith R and Galloway M. Boat orientation and skill level in sculling boats. In: XVIII International Symposium on Biomechanics in Sports, The Chinese University of Hong Kong, Hong Kong, June 252000.

29. Wing AM and Woodburn C. The coordination and consistency of rowers in a racing eight. Journal of Sports Science 1995; 13: 187-197.

30. Kinoshita T, Miyashita M, Kobayashi $\mathrm{H}$ and Hino T. Rowing Velocity Prediction Program with Estimating Hydrodynamic Load Acting on an Oar Blade. In: Kato $\mathrm{N}$ and Kamimura S (eds) Bio-mechanisms of Swimming and Flying: Fluid Dynamics, Biomimetic Robots, and Sports Science. Japan: Springer, 2008, pp.345-359. 


\section{Chapter 3}

\section{Validity and Reliability of the PowerLine Rowing Instrumentation System}

\subsection{Introduction}

The PowerLine (PL) Rowing Instrumentation System features replacement oarlocks that include strain gauge load cells, which quantify force at $50 \mathrm{~Hz}$. The load cell consists of three concentric tubes connected in series [1]. The inner tube fits onto the pin of a wing rigger and has a locking mechanism that prevents its rotation around the pin [1]. A swivel fits onto the outer tube of the load cell and can rotate freely; four strain gauges are bound to the middle tube [1]. Individual strain gauges work on the concept of electrical resistance $R_{e}$,

$R_{e}=\rho \frac{L_{g}}{A}$

where $\rho$ represents the material's electrical resistivity, $L_{g}$ is the length of the gauge and $A$ is its cross-sectional area [2]. When force is applied to an instrumented oarlock, its material deforms, the gauge responds to the elastic deformation of the oarlock's material (i.e., by changing $L_{g}$ and $A$ ) and the electrical resistance $R_{e}$ changes. "Gauge factors" are used to relate the changes in $R_{e}$ to changes in strain. Measures of strain from individual strain gauges are temperature sensitive because thermal expansion can affect the volume of matter in the material of the gauge and in the material of the object to which the strain gauge is attached [2]. The PL oarlocks minimize their sensitivity to changes in temperature by connecting the individual strain gauges in a Wheatstone bridge circuit [1].

According to Haines [1], the strain gauges in the PL oarlocks are configured to measure the forces applied in the direction parallel to the boat's 
main motion. In other words, the PL oarlocks are engineered to be insensitive to the forces applied in the orthogonal and vertical directions. The PL force measurements were originally sensitive to the location of the point of force application [1]. Accordingly, the PL oarlocks were reengineered to use voltage outputs from two Wheatstone half-bridges to estimate the location of an applied force on the face of the PL swivel and automatically calibrate the force measurements [1]. The PL force measurements have an engineering tolerance of $\pm 2 \%$ of the force measurement [3].

Many national rowing programs use the PL system including Great Britain, South Africa, Brazil, New Zealand, France, Denmark, Netherlands, United States and Canada [3]. Despite its global popularity, only one independent study has investigated the validity of the PL force measurements [4]. Dynamic forces of up to $554.8 \pm 20.4 \mathrm{~N}$ were manually applied to a loading bar that was suspended from the PL oarlocks with a load cell linked in series. The results of a linear regression analysis indicated excellent agreement between the $\mathrm{PL}$ and load cell measurements. The authors concluded that "the validity of the PL measurements were acceptable over the range tested in the laboratory" [4]. However, the consistency of the PL force measurements over time has not been established, and previous research [4] has only tested the accuracy of PL scull oarlocks. Therefore, the following work investigates the convergent validity and test-retest reliability of the force measurements from sweep and scull PL oarlocks.

\subsection{Methods}

Seventeen PL oarlocks ( $n=17$ ), nine sweep and eight scull, were stored and tested in a laboratory with a regulated room temperature of $22 \pm 2{ }^{\circ} \mathrm{C}$. The oarlock's angle was measured in order to resolve the forces on the PL oarlocks that represent those that act in the $x$-direction. The angular displacement of the $P L$ swivel is measured relative to the inner tube through two Hall effect sensors and an 8-axial pole ring magnet [1]. The angle measurements can also be determined relative to the rowing boat because the inner tube secures to the pin 
in a fixed direction (i.e., in the $y$-axis). The PL angle measurements have $a \pm 0.5^{\circ}$ tolerance [3].

The inner tubes of the PL oarlocks were secured to a cylindrical bar that was supported by two squat stands; the bar represents the pin on a wing rigger. The bases of the inner tubes were orientated perpendicular to the PL swivels using a spirit level (Figure 12). The PL swivels were pointed in the $x$-direction and the bases of the inner tubes were pointed in the $y$-direction. Through this perpendicular orientation, any mass suspended from the $\mathrm{PL}$ swivel will act in a direction that represents the direction of the boat's main motion. Connection cables linked the PL oarlocks to a programmable data-logger that displayed the real-time measurements. With the perpendicular orientation between the inner tubes and the PL swivels, the force and angle measurements were zeroed through the data-logger's local interface.

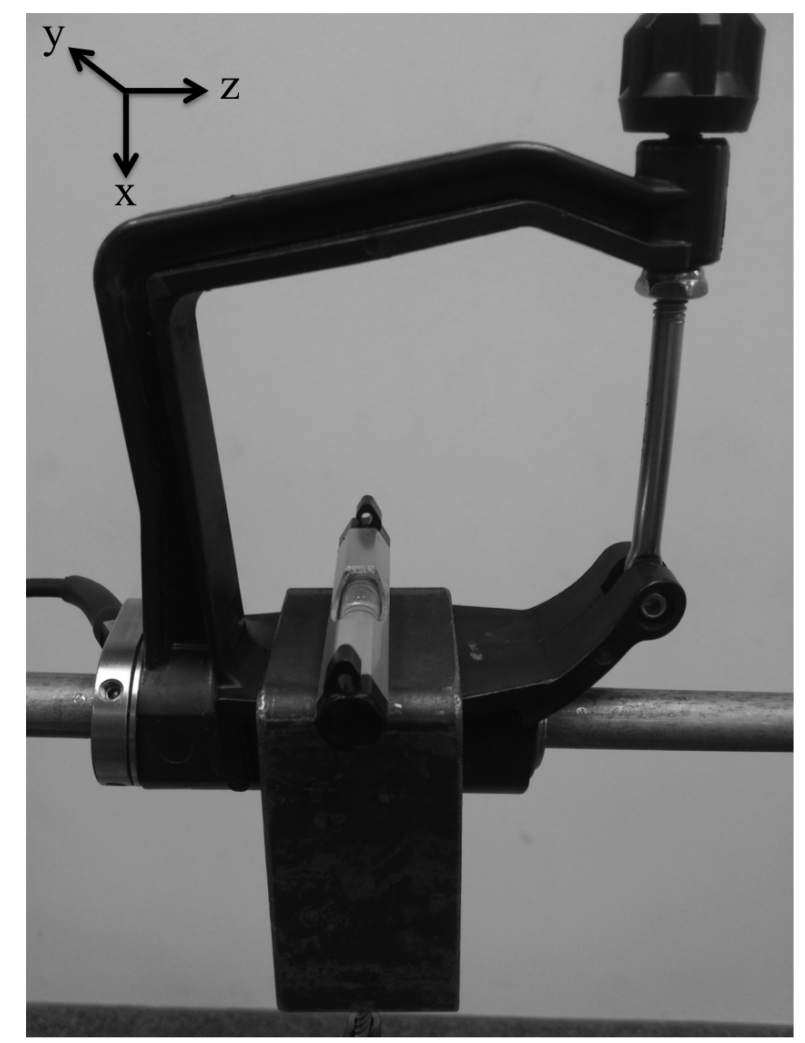

Figure 12. Orientating the PL swivel perpendicular to the base of the inner tube (shown in the left side of the photograph) using a spirit level. 
Static forces of $0,32.4,255.1$ and $431.6 \mathrm{~N}$ were individually applied to the PL oarlocks using a custom-made suspension rig that was loaded with weight plates. The suspension rig consisted of a box, wire cable and a loading bar connected in series (Figure 13). The weights of the plates and the suspension rig were measured using a digital bench scale (Rice Lake Weighing Systems, Wisconsin, United States) with a $\pm 0.98 \mathrm{~N}$ tolerance. $0 \mathrm{~N}$ is the theoretical force on the oarlock when it points in the $x$-direction, $32.4 \mathrm{~N}$ is the weight of the suspension rig, and 255.1 and $431.6 \mathrm{~N}$ are the weights of the plates, which includes the weight of the suspension rig.

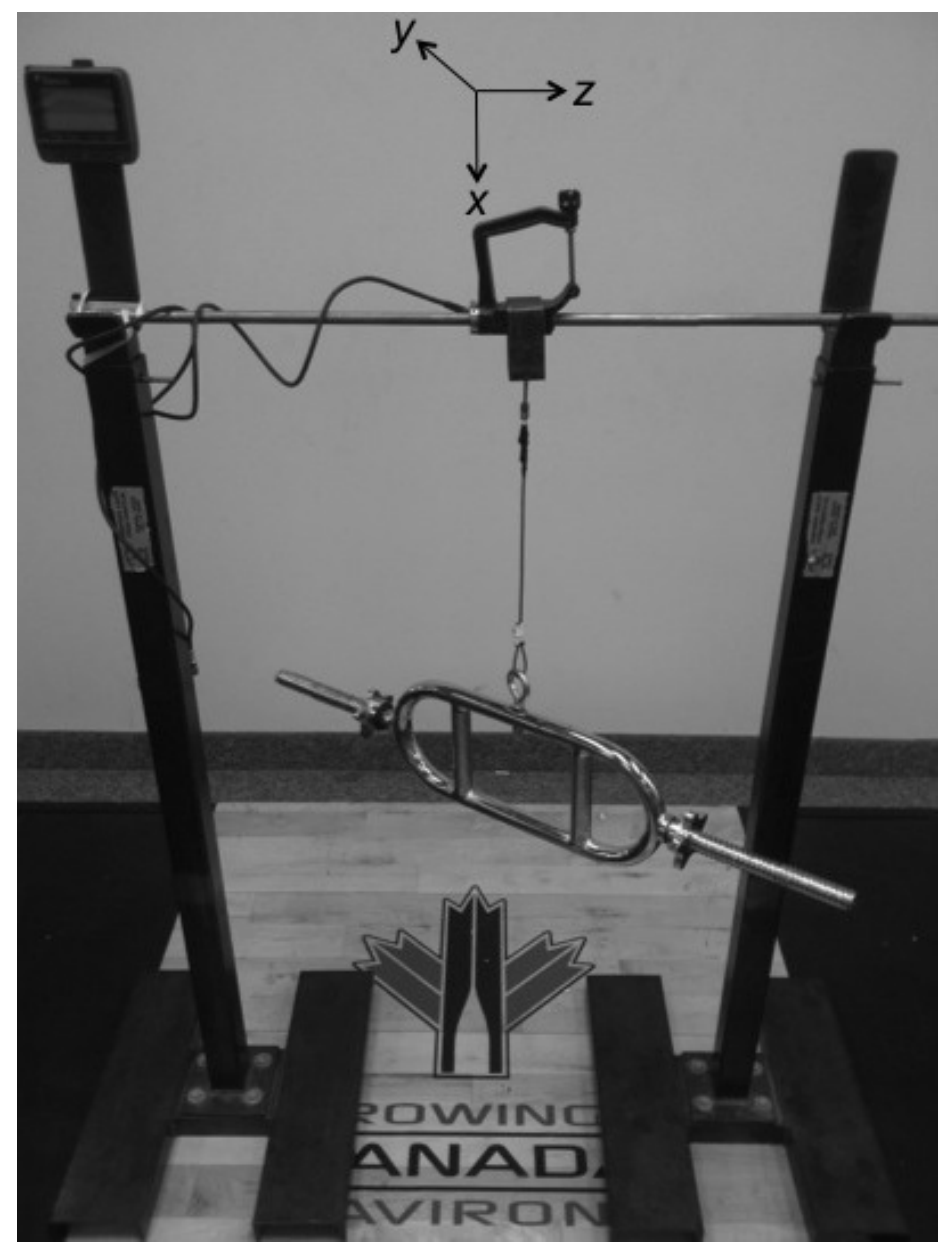

Figure 13. Photograph of the experimental setup used to test the PL oarlocks. A bar is supported by two stands, and a PL oarlock is fixed to the bar. The PL swivel is pointed in the $x$-direction and the base of the inner tube is pointed in the $y$-direction. The PL oarlock is connected to a data-logger, and a suspension rig is hanging from the oarlock. 
The PL force measurements slightly fluctuate while the oarlocks are statically loaded - this was described as random error. Random error is inherently unpredictable fluctuations in a measuring instrument [5], and can be reduced through calculating the arithmetic mean of multiple measurements [6]. The PL oarlocks were statically loaded for five seconds and the mean force measurement over that period was calculated and used in the analysis. Note that for a typical rowing drive, the duration of force application from the oar to the oarlock is approximately one second [7]. Data were collected over fifteen days and statistically analyzed using SPSS Statistics Version 21 (IBM Corp., Ontario, Canada). The statistical significance was set to .05 , and the results are presented with $95 \%$ confidence.

\subsection{Results}

\subsubsection{Test-Retest Reliability}

Test-retest reliability is the consistency of an instrument to reproduce similar measurements over time [8]. The distributions of the PL force measurements were examined for normality and homogeneity of variance. Normality refers to a theoretical frequency distribution that is "bell-shaped" and symmetric about the mean [9]. A Shapiro-Wilk test [10] was used to test for normality in the distributions. The null hypothesis $H_{0}$ is that the PL force measurements are normally distributed as a function of the testing date. The $p$-values are shown in Table 4 . Since the majority of $p$-values were $<.05$, this suggests that the results are inconsistent with the assumption that the $H_{0}$ is true, and thus it must be rejected. 
Table 4. Analyzing normality of the PL force measurements as a function of the testing date using a Shapiro-Wilk test.

\begin{tabular}{ccc}
\hline Testing Date & Sweep $p$-values & Scull $p$-values \\
\hline 1 & .000 & .000 \\
2 & $.269^{*}$ & .020 \\
3 & .018 & .000 \\
4 & .008 & .000 \\
5 & .001 & .000 \\
6 & $.357^{*}$ & .002 \\
7 & .045 & .005 \\
8 & .000 & .002 \\
9 & .002 & .037 \\
10 & .000 & .004 \\
11 & .005 & $.707^{*}$ \\
12 & .008 & .028 \\
13 & $.054^{*}$ & .000 \\
14 & .020 & .046 \\
15 & .007 & .030 \\
\hline
\end{tabular}

Note: If the $p$-value is $<.05$, the results reject the $H_{0}$. An asterisk * indicates a normal distribution $(p>.05)$.

Since the results were statistically significant, a non-parametric Levene's F-test [11] was used to test for homogeneity of variance in the PL force measurements over the fifteen days of testing. Data is homoscedastic if all variables in a sample have similar variance [12]. In contrast, heteroscedasticity is when the variables in a sample have different variance [12]. The $H_{0}$ is that there is homogeneity of variance. The $p$-values for sweep and scull oarlocks were .203 and .142 , respectively. Since the $p$-values were $>.05$, the results fail to reject the $H_{0}$. This indicates homogeneity of variance in the distributions of the PL force 
measurements as a function of the testing date. Although a parametric analysis of variance (ANOVA) can be robust to violations of normality [13], a nonparametric model was selected to provide a more conservative analysis of the test-retest reliability of the PL force measurements. The reduced statistical power associated with non-parametric models was considered.

A Kruskal-Wallis One-Way ANOVA was used to determine the test-retest reliability of the PL force measurements over the fifteen days of testing; the analysis does not assume a normal distribution. The differences $F_{\text {diff }}$ between the $\mathrm{PL}$ force measurements and the known static forces were calculated. The independent variable was the testing date and the dependant variable was the $F_{\text {diff. }}$ The $H_{o}$ is that there is no difference in the $F_{\text {diff }}$ over the fifteen days of testing. The $p$-values were .335 for scull and .451 for sweep oarlocks. Since the $p$-values were $>.05$, the results fail to reject the $H_{0}$. This suggests that the PL force measurements were consistent over the fifteen days of testing. The maximum differences in the PL force measurements over the fifteen days of testing when loaded with $431.6 \mathrm{~N}$, for instance, were $18.8 \pm 11.9 \mathrm{~N}$ for sweep and $16.8 \pm 6.2 \mathrm{~N}$ for scull oarlocks; the uncertainties are SD.

\subsubsection{Convergent Validity}

Convergent validity is the observed correlation between two independent measures that theoretically correlate [8]. Shapiro-Wilk tests [10] and histogram plots were used to examine the distributions of the PL force measurements as a function of the known static forces (Table 5). The mean PL force measurements, obtained from the histogram plots, were at least $97.2 \%$ of the values of the known static forces. Excluding the baseline measurements at $0 \mathrm{~N}$, the variations in the distributions (i.e., shown in the uncertainty) ranged from 1.2 to $5.7 \%$ of the mean PL force measurements. These variations can be attributed to round-off errors or limitations in the PL oarlock's sampling rate. The distributions were statistically analyzed for normality using a Shapiro-Wilk test [10]. The $H_{0}$ is that the PL force measurements are normally distributed as a function of the known 
static forces. Since the majority of $p$-values were $<.05$ (Table 5 ), the results reject the $H_{0}$. This indicates that the PL force measurements are not normally distributed as a function of the known static forces. A non-parametric Levene's Ftest [11] was used to test for homogeneity of variance in the $P L$ force measurements as a function of the known static forces. The $H_{0}$ is that there is homogeneity of variance in the distributions. The $p$-values for sweep and scull oarlocks were both .100 . Since the $p$-values were $>.05$, the results fail to reject the $H_{0}$. This indicates homogeneity of variance in the PL force measurements over the range of forces that were tested.

Table 5. Investigating normality of the PL force measurements as a function of the known static forces using a Shapiro-Wilk test.

\begin{tabular}{ccccc}
\hline $\begin{array}{c}\text { Known Force } \\
(\mathrm{N})\end{array}$ & $\begin{array}{c}\text { Sweep } \\
\text { Force }(\mathrm{N})\end{array}$ & Sweep p-value & $\begin{array}{c}\text { Scull } \\
\text { Force }(\mathrm{N})\end{array}$ & Scull p-value \\
\hline 0.0 & $0.1 \pm 0.1$ & .000 & $0.1 \pm 0.1$ & .000 \\
32.4 & $31.5 \pm 1.8$ & .000 & $31.7 \pm 1.6$ & $.100^{*}$ \\
255.1 & $250.7 \pm 3.6$ & .016 & $249.9 \pm 4.1$ & .000 \\
431.6 & $425.8 \pm 5.5$ & .000 & $425.3 \pm 5.1$ & .043 \\
\hline
\end{tabular}

Note: If the $p$-value is $<.05$, the results reject the $H_{0}$. An asterisk * indicates a normal distribution $(p>.05)$. The PL force measurements for all sweep and scull oarlocks, combined over the fifteen days of testing, are presented as the mean \pm SD for each load.

Since the data violated the parametric assumption of normality, a Wilcoxon One-Sample Signed Rank Test was used to analyze the convergent validity of the PL force measurements. The Wilcoxon One-Sample Signed Rank Test is a non-parametric statistic, which examines the relationship between a measured sample median and a pre-determined hypothesized median [13]. The known static forces represent the hypothesized medians and the PL force measurements are the measured medians. The $H_{0}$ is that the median PL force measurements are equal to the values of the known static forces. Typical results 
are shown in Table 6. Although the median PL force measurements were $98.1 \%$ \pm 0.8 percentage points $(\mathrm{pp})$ of the values of the known static forces, the results rejected the $H_{o}$ since the $p$-values were $<.05$. This is considered Type 1 error since the results rejected the $H_{o}$ when, in reality, it was true. From this point forward, the convergent validity of the PL force measurements will be discussed numerically. Excluding the baseline measurements at $0 \mathrm{~N}$, the median PL force measurements were slightly less than the values of the known static forces (i.e., $2.0 \% \pm 0.8 \mathrm{pp}$ ). The maximum differences between the PL force measurements and the known static forces were $15 \pm 4 \mathrm{~N}$ for scull and $14 \pm 7 \mathrm{~N}$ for sweep oarlocks.

Table 6. Testing the convergent validity of the PL force measurements using a Wilcoxon One-Sample Signed Rank Test.

\begin{tabular}{ccccc} 
Known Force (N) & $\begin{array}{c}\text { Median Sweep } \\
\text { Force (N) }\end{array}$ & Sweep p-value & $\begin{array}{c}\text { Median Scull } \\
\text { Force (N) }\end{array}$ & Scull $p$-value \\
\hline 0.0 & 0.1 & .000 & 0.1 & .000 \\
32.4 & 31.3 & .000 & 31.8 & .000 \\
255.1 & 250.2 & .000 & 250.2 & .000 \\
431.6 & 425.6 & .000 & 426.2 & .000 \\
\hline
\end{tabular}

Note: If the $p$-value is $<.05$, the results reject the $H_{0}$.

One scull and sweep oarlock were used to test whether the PL force measurements, for a constant load, depend on the point of force application on the face of the PL swivel. The box of the suspension rig, while loaded with 255.1 $\mathrm{N}$, was translated along the face of the PL swivels in the z-axis. Anecdotal results showed that the differences in the PL force measurements as a function of the point of force application were on the same order of magnitude as the small fluctuations in the PL force measurements associated with random error. 


\subsubsection{Calibration Factors}

Calibration factors for each PL oarlock were established to correct for the slight discrepancies in the force measurements. Figure 14 shows an example of the PL force measurements as a function of the known static forces; the other PL oarlocks showed a similar trend. There are no visual signs of heteroscedasticity, which is in agreement with the aforementioned results from the non-parametric Levene's F-tests. A linear model was fit to the data using a least squares linear regression analysis generated in MATLAB 2013a (The MathWorks Inc., Massachusetts, USA). Though not shown here, the residuals were scattered randomly about the zero point.

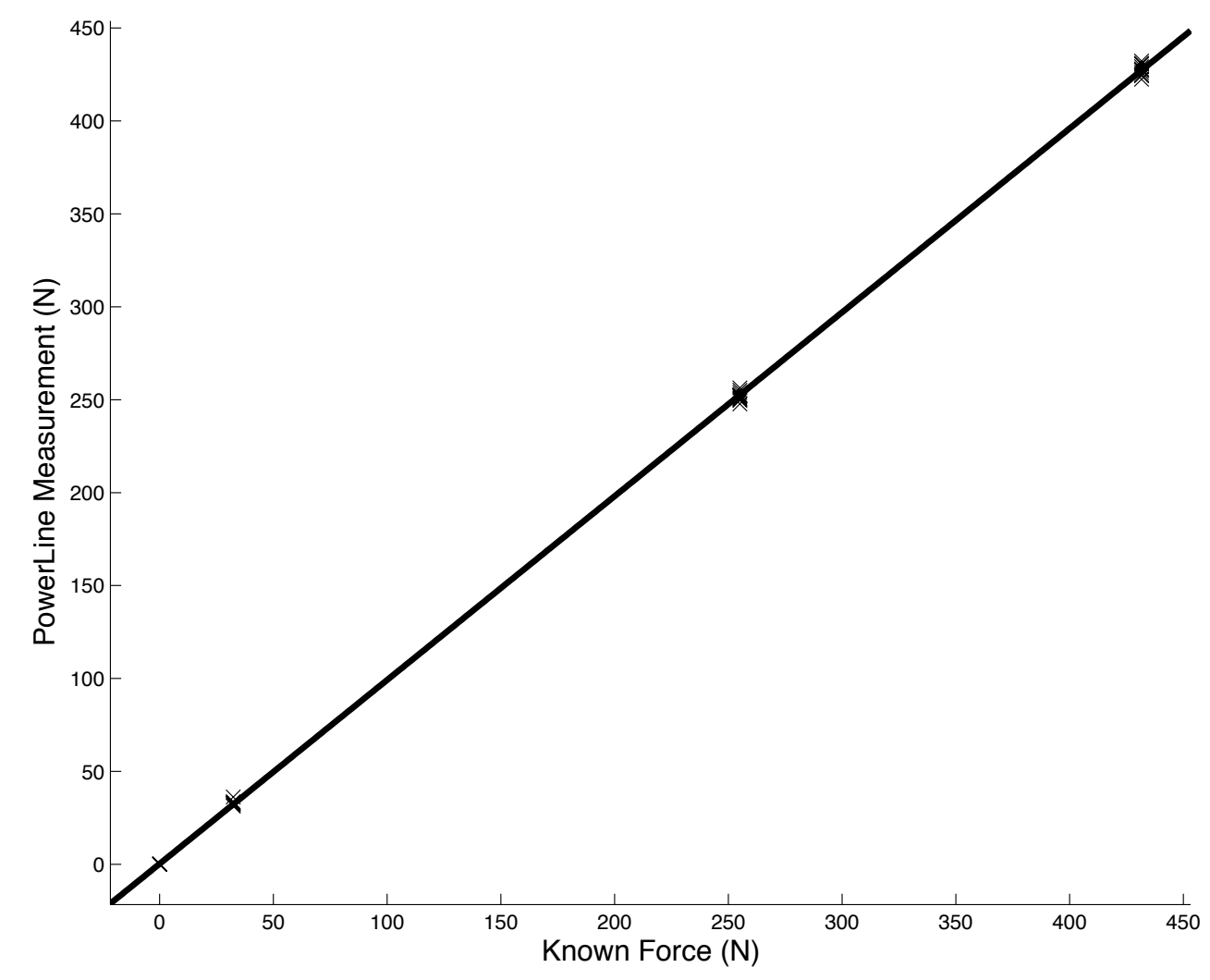

Figure 14. The PL force measurements as a function of the known static forces for a single sculling oarlock. The linear fit is a regression line. 
Table 7 shows the slope, coefficient of determination $\left(R^{2}\right)$ and $y$-intercept for the PL force measurements as a function of the known static forces. The $R^{2}$ quantifies how well the linear regression fits the data [14]. The linear regressions accurately fit the data with $R^{2} \geq .999$. In a calibration experiment, the linear regression should ideally pass through the origin [14]. For the analysis, the $H_{0}$ is that there is no difference between the $y$-intercept and the origin. Since the $p$ values were $>.05$ (Table 7), the results fail to reject the $H_{0}$. This indicates that the $y$-intercepts passed through the origin. The slope value for each PL oarlock is its calibration factor. For example, a slope of .982 indicates that, on average, applying a known force of $1 \mathrm{~N}$ will correspond to a model prediction of a PL force measurement of $0.982 \mathrm{~N}$. The slopes ranged from .976 to .993 , which indicates that the PL oarlocks underestimated the applied forces. 
Table 7. The slope, $y$-intercept and $\mathrm{R}^{2}$ for the $\mathrm{PL}$ force measurements as a function of the known static forces from a least-squares linear regression analysis.

\begin{tabular}{|c|c|c|c|c|c|}
\hline Type & Oarlock ID & Slope & $y$-intercept & $\begin{array}{c}y \text {-intercept } \\
p \text {-value }\end{array}$ & $\mathrm{R}^{2}$ \\
\hline \multirow[t]{9}{*}{ Sweep } & 2664 & $.989 \pm .003$ & $-.18 \pm .88$ & .837 & .999 \\
\hline & 2442 & $.982 \pm .002$ & $-.52 \pm .47$ & .273 & 1 \\
\hline & 2441 & $.985 \pm .001$ & $-.32 \pm .38$ & .396 & 1 \\
\hline & 2435 & $.985 \pm .003$ & $-1.08 \pm .76$ & .161 & .999 \\
\hline & 2443 & $.983 \pm .003$ & $.39 \pm .84$ & .644 & .999 \\
\hline & 3214 & $.991 \pm .002$ & $-.08 \pm .53$ & .880 & 1 \\
\hline & 3215 & $.991 \pm .002$ & $-.07 \pm .41$ & .869 & 1 \\
\hline & 2665 & $.993 \pm .002$ & $-.38 \pm .40$ & .338 & 1 \\
\hline & 2299 & $.978 \pm .002$ & -.68 .47 & .157 & 1 \\
\hline \multirow[t]{8}{*}{ Scull } & 2305 & $.989 \pm .001$ & $.24 \pm .36$ & .511 & 1 \\
\hline & 2444 & $.990 \pm .002$ & $-.38 \pm .66$ & .570 & 1 \\
\hline & 2445 & $.987 \pm .003$ & $-.16 \pm .70$ & .818 & .999 \\
\hline & 2307 & $.982 \pm .002$ & $.18 \pm .55$ & .752 & .999 \\
\hline & 2447 & $.976 \pm .003$ & $-.75 \pm .78$ & .343 & .999 \\
\hline & 3646 & $.980 \pm .002$ & $.03 \pm .60$ & .960 & 1 \\
\hline & 2446 & $.984 \pm .002$ & $-.87 \pm .44$ & .055 & 1 \\
\hline & 2306 & $.989 \pm .002$ & $-.53 \pm .53$ & .329 & 1 \\
\hline
\end{tabular}

Note: The slope and $y$-intercepts for each PL oarlock are expressed as the coefficient \pm SD. If the $p$-value is $>.05$, the results fail to reject the $H_{0}$.

\subsection{Discussion}

The day-to-day consistency of the PL force measurements has not been formerly documented. Inter-day differences in force measurements from strain gauge 
based technology, outside of human error, have been largely attributed to changes in temperature, pressure and humidity [15]. Considering that the PL oarlocks were stored and tested in a laboratory with a regulated room temperature, it is somewhat expected that the PL force measurements were consistent over the fifteen days of testing. Since rowers compete on-water in a wide variety of weather conditions, investigating the test-retest reliability of the PL force measurements in an outdoor setting presents an interesting topic for future research.

The differences between the PL force measurements and the known static forces were at most $15 \pm 4 \mathrm{~N}$ for scull and $14 \pm 7 \mathrm{~N}$ for sweep oarlocks. These findings show that the PL force measurements are more accurate than that originally proposed by Coker et al. [4], who reported maximum differences of 15.5 to $45.6 \mathrm{~N}$ between a load cell and the PL oarlocks. Maximum oarlock forces for an elite heavyweight female rower, averaged over 20 strokes at a rate of 20 strokes/min, are purportedly $807.2 \pm 79.4 \mathrm{~N}$ in sweep and $449.9 \pm 10.1 \mathrm{~N}$ in sculling (Canadian Sport Institute Ontario, 2014, personal communication). Therefore, the inter-stroke inconsistencies (i.e., shown in the uncertainties) in the maximum oarlock forces for an elite rower were generally the same as or larger than the maximum differences between the PL force measurements and the independent measures of force observed in this chapter and in previous research [4].

The maximum forces used in the laboratory tests were 95 and $53 \%$ of the maximum forces previously measured on scull and sweep oarlocks for an elite heavyweight rower during on-water rowing (Canadian Sport Institute Ontario, 2014, personal communication). These differences in maximum force are not considered a limitation of this work because the convergent validity of the PL force measurements was independent to the magnitude of the applied static forces. It is projected that suspending heavier loads would yield similar results. 
The PL force measurements are supposedly insensitive to the location of the point of force application [1]. This was assessed by translating the box of the suspension rig along the face of two PL swivels in the z-axis while loaded with a constant force. The differences in the PL force measurements as a function of the point of force application were on the same order of magnitude as the small fluctuations in the PL force measurements associated with random error.

Total error in a measuring instrument consists of both random error, as previously described, and systematic error $[5,16]$. Systematic error refers to predictable measurement errors that consistently differ from a known value [5, 16]. Systematic error in a measuring instrument can result from zero error (i.e., also known as off-set error). Zero error is when an instrument does not measure zero when the known quantity is zero [6]; this can "off-set" the $y$-intercept from the origin. Imperfect zeroing of a measuring instrument is generally the cause of zero error [6]. The results show that the $y$-intercepts passed through the origin, which indicates that the PL oarlocks measured approximately $0 \mathrm{~N}$ when the known static force was $0 \mathrm{~N}$. These results support the accuracy of the zeroing protocol used.

Calibration factors can be applied to a set of measurements to compensate for bias associated with systematic error [17]. What remains, after the calibration factors have been applied, are the uncertainties associated with systematic errors [17]. The slopes ranged from .976 to .993 . These results indicate that the PL oarlocks slightly underestimated the applied forces, and that the uncertainties associated with systematic error were relatively small (i.e., 0.7 to $2.4 \%$ ). The slope and $R^{2}$ strongly agreed with those from previous research [4], which reported slopes of $1.01 \pm .04$ and $R^{2}$ of $.999 \pm .004$. In conclusion, the calibration factors produced in this chapter can be applied to the PL force measurements to correct for the slight discrepancies. 


\subsection{References}

1. Haines P. Force-sensing system. Patent 7114398 B2, USA, 2004.

2. Blake A. Handbook of Mechanics, Materials, and Structures. USA: John Wiley \& Sons, 1985.

3. Peach Innovations. PowerLine Rowing Instrumentation System, www.peachinnovations.com (assessed 11 June 2014).

4. Coker J, Hume P and Nolte V. Validity of the PowerLine Boat Instrumentation System. In: Scientific Proceedings of the 27th International Conference on Biomechanics in Sports (ed R Anderson, D Harrison and I Kenny), University of Limerick, Ireland, August 17-21 2009, pp.65-68. International Society of Biomechanics in Sports.

5. American Society of Mechanical Engineers. Test Uncertainty. Performance Test Code 19.1, 2005.

6. Singh M. Introduction to Biomedical Instrumentation. India: PHI Learning Private Limited, 2010.

7. Baca A and Kornfeind P. A Feedback System for Coordination Training in Double Rowing. In: Estivalet M and Brisson P (eds) The Engineering of Sport 7: Volume 1. France: Springer, 2009, pp.659-668.

8. Kimberlin CL and Winterstein AG. Validity and reliability of measurement instruments used in research. American Journal of Health-System Pharmacy 2008; 65: 2276-2284.

9. Peck R, Olsen C and Devore J. Introduction to Statistics and Data Analysis (4 ${ }^{\text {th }}$ ed). USA: Cengage Learning, 2011.

10. Shapiro SS and Wilk MB. An Analysis of Variance Test for Normality (Complete Samples). Biometrika 1965; 52: 591-611. 
11. Nordstokke DW and Zumbo BD. A new nonparametric Levene test for equal variances. Psicológica 2010; 31: 401-430.

12. Sheskin DJ. Handbook of Parametric and Nonparametric Statistical Procedures ( $3^{\text {rd }}$ ed). USA: Chapman \& Hall/CRC, 2004.

13. Schmider E, Ziegler M, Danay E, Beyer $L$ and Bühner $M$. Is it really robust? Reinvestigating the robustness of ANOVA against violations of the normal distribution assumption. European Journal of Research Methods for the Behavioural and Social Sciences 2010; 6: 147-151.

14. Barwick V. Preparation of Calibration Curves: A Guide to Best Practice. Report, National Measurement System Valid Analytical Measurement Programme, UK, 2003.

15. Steinchen W and Yang L. Digital Shearography: Theory and Application of Digital Speckle Pattern Shearing Interferometry. USA: The Society of PhotoOptical Instrumentation Engineering, 2003.

16. Atkinson $G$ and Nevill AM. Statistical Methods For Assessing Measurement Error (Reliability) in Variables Relevant to Sports Medicine. Sports Medicine 1998; 26: 217-238.

17. Kirkup L and Frenkel RB. An Introduction to Uncertainty in Measurement: Using the GUM (Guide to the Expression of Uncertainty in Measurement). UK: Cambridge University Press, 2006. 


\section{Chapter 4}

\section{Rowing with Different Oar-Shafts}

\subsection{Introduction}

The effect of oar-shaft stiffness on rowing biomechanics is not well known. Many previous studies have assumed that the oar-shaft is perfectly rigid [1-11]. The dynamic behaviour of the oar-shaft during the drive is illustrated schematically in Figure 15. The equilibrium position $E_{x}$ is the point where the magnitude of the oar-shaft's deflection in the $x$-axis is zero (i.e., during the recovery when there is no load on the blades - neglecting air resistance). Following the catch position, the blades enter the water and the rower pulls on the handles. The oar-shafts deflect $\delta$ towards the bow as the blades experience load while moving through the water. This deflection stores elastic potential energy in the shaft's material. Towards the end of the drive, the rower's force application to the handles decreases and the oar-shaft's inversely deflect $\delta^{-1}$ back to their $E_{x}$ position (Figure 15).

Less stiff oar-shafts presumably deflect more than stiffer oars during the drive, and thus store more elastic potential energy. Some rowing enthusiasts claim that the amount of elastic energy stored in less stiff oar-shafts is large enough that, when transformed back to kinetic energy, the oar-shafts inversely deflect at a rate that could increase boat acceleration [12]. However, the water provides a damping effect on the blade's movement via viscous drag, thus reducing the likelihood of the less stiff oar-shafts inversely deflecting at high enough rates to generate propulsive effects. In addition, a portion of the mechanical energy will dissipate as thermal energy since on-water rowing is not an isolated system. 


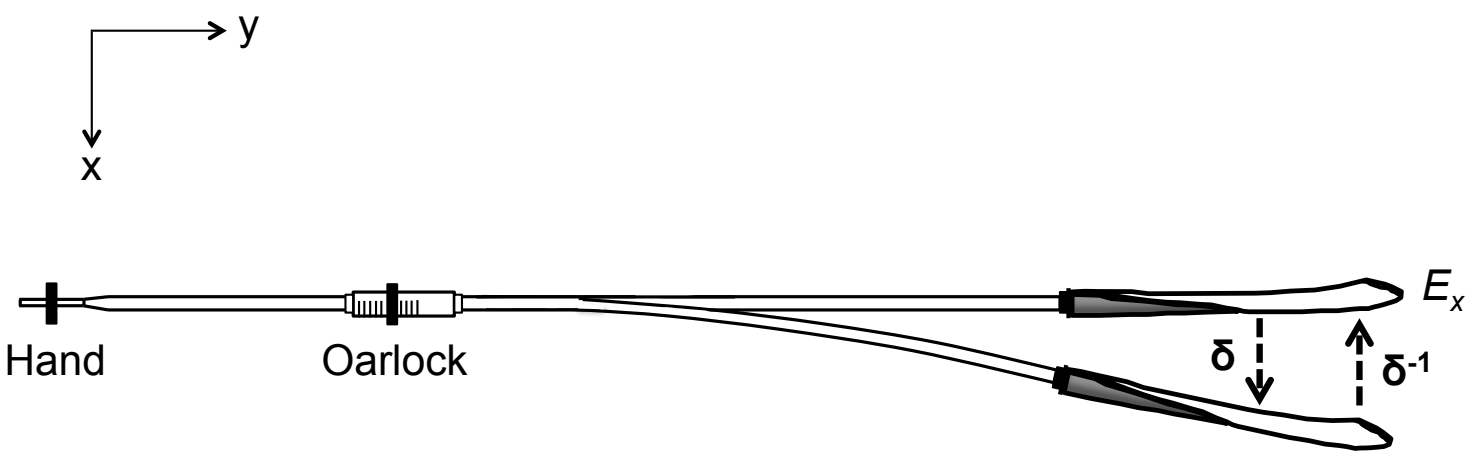

Figure 15. Schematic of the oar-shaft's dynamic behavior during the drive. The equilibrium position $E_{x}$ refers to the point where the magnitude of the oar-shaft's deflection in the $x$-axis is zero. $\delta$ is deflection and $\delta^{-1}$ is inverse deflection, as described in the text. Deflection of the inboard is neglected in this model.

Since an oar-shaft's stiffness will effect its deflection during the drive, it also has implications on the blade's AOA. To recall, the AOA is the angle between the blade's reference line and the vector representing the oncoming flow of water. The AOA indirectly affects the hydrodynamic lift $\mathbf{F}_{\mathbf{I}}$ and drag $\mathbf{F}_{\mathbf{d}}$ forces on the blades [13], which are calculated via

$$
\begin{aligned}
& \mathbf{F}_{\mathbf{l}}=1 / 2 \mathrm{C}_{\mid} \rho_{\mathrm{w}} A^{\prime} \boldsymbol{v}^{2} \\
& \mathbf{F}_{\mathrm{d}}=1 / 2 \mathrm{C}_{\mathrm{d}} \rho_{\mathrm{w}} A^{\prime} \mathbf{v}^{2}
\end{aligned}
$$

where $\rho_{w}$ is the water density, $A^{\prime}$ is the blade's reference area, $c_{d}$ and $c_{l}$ are dimensionless drag and lift coefficients, and $\boldsymbol{v}$ is the resultant velocity of the blade relative to the water. The blade's geometry and AOA affect $c_{d}$ and $c_{l}[13]$. An oar-shaft's stiffness may affect the AOA and $A^{\prime}$ during the drive, and thus change the hydrodynamic forces on the blade. Hofmijster et al. [14] investigated this using both theoretical and experimental techniques, which are described in Chapter 2. The authors found that assuming a perfectly rigid oar-shaft changed the reconstructed blade kinematics during the drive, which changed the hydrodynamic forces calculated on the blades [14].

The effects of oar length on rowing biomechanics are also of interest. The external forces that act on the rowing oar during the drive are commonly 
illustrated using a lever model (Figure 16). $\mathbf{F}_{\mathbf{h}}$ represents the effort applied by the rower to the handle, $\mathbf{F}_{\mathbf{b}}$ is the load on the blade, and $\mathbf{F}_{\mathbf{o}}$ is the normal reaction force at the oarlock, which is the sum of $\mathbf{F}_{\mathrm{b}}$ and $\mathbf{F}_{\mathrm{h}}$. The lines of action are all modelled in the $x$-axis. The support moment arm $L_{s}{ }^{\prime}$ is the perpendicular distance between the points of application of the force vectors $\mathbf{F}_{\mathbf{h}}$ and $\mathbf{F}_{\mathbf{o}}$, and the beam moment arm $L_{b}{ }^{\prime}$ is the perpendicular distance between the points of application of vectors $\mathbf{F}_{\mathbf{o}}$ and $\mathbf{F}_{\mathbf{b}}$.

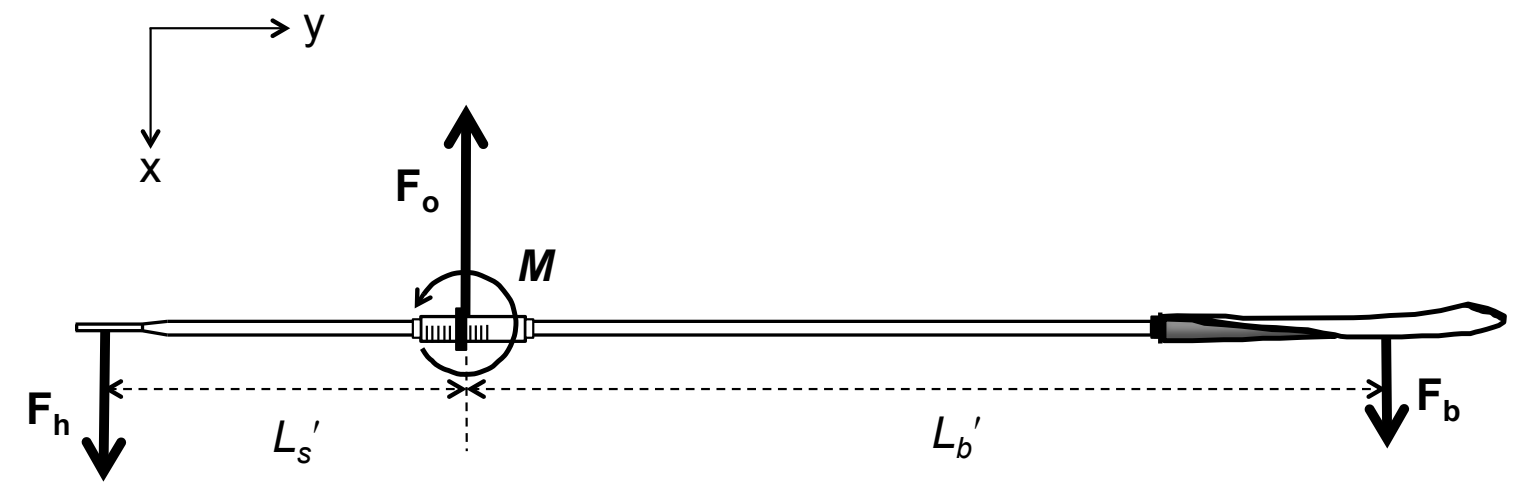

Figure 16. Free body diagram of the external forces that act on the rowing oar during the drive. $\mathbf{F}_{\mathrm{h}}$ is the force applied by the rower to the handle, $\mathbf{F}_{\mathrm{o}}$ is the normal reaction force at the oarlock, $\boldsymbol{M}$ is the resultant moment of force, $\mathbf{F}_{\mathbf{b}}$ is the load on the blade, $L_{b}{ }^{\prime}$ is the beam moment arm, and $L_{s}{ }^{\prime}$ is the support moment arm.

The moment of force about the oarlock $\boldsymbol{M}$ (i.e., the fulcrum) in dynamic equilibrium is calculated [13] via

$\sum \boldsymbol{M}=\boldsymbol{F}_{\boldsymbol{h}} L_{s}{ }^{\prime}-\boldsymbol{F}_{\boldsymbol{b}} L_{b}{ }^{\prime}-I_{m} \boldsymbol{\alpha}=0$

where $\boldsymbol{\alpha}$ is the angular acceleration of the oar and $I_{m}$ is its mass moment of inertia. Nolte [13] presented evidence that suggests $I_{m} \boldsymbol{\alpha}$ can be neglected in most cases, as it is relatively small compared to the other terms in the equation. Therefore, equation (7) can be rewritten as

$\boldsymbol{F}_{\boldsymbol{h}} L_{s}{ }^{\prime}=\boldsymbol{F}_{\boldsymbol{b}} L_{b}{ }^{\prime}$ 
Assuming a hydrodynamically efficient blade design, Nolte [13] estimated that shorter oars are more effective in rowing since a shorter $L_{b}{ }^{\prime}$ could produce larger $\mathbf{F}_{\mathbf{b}}$ for a given $\mathbf{F}_{\mathbf{h}}$ and $L_{s}{ }^{\prime}$. However, the rowing oar will not likely yield an ideal mechanical advantage since there is friction between the oarlock and pin, and because the oar-shaft deflects as the blade moves through the water. In addition, recent work using computational fluid dynamics has reported that the location of the point of force application on the blade varies with respect to drive time [15]. Therefore, treating $\mathbf{F}_{\mathbf{b}}$ as a constant force that acts at a fixed distance $L_{b}{ }^{\prime}$ from the collar may be unrealistic.

Previous studies that have considered the effects of oar-shaft stiffness $[14,16]$ and length [13] on rowing performance have been largely theoretical. In contrast, the following work experimentally measures the biomechanics of rowing with oar-shafts of different stiffness and length, and discusses the results with relation to oar-shaft deflection, inverse deflection, and lever theory.

\subsection{Methods}

\subsubsection{Participants}

Four female rowers (mean $\pm \mathrm{SD}$ : age $=22 \pm 3$ years, mass $=60.1 \pm 1.2 \mathrm{~kg}$, and height $=1.69 \pm 0.03 \mathrm{~m}$ ) were recruited from the University of Western Ontario's varsity program. Previous research with similar objectives used smaller sample sizes $[6,14,17]$. The rowers gave informed written consent to participate. The University of Western Ontario Research Ethics Board for Health Sciences Research involving Human Subjects approved this work (Appendix 1).

\subsubsection{Materials}

The same oars previously investigated in Chapter 2 were used in this experiment. To recall, the two sets of sculling oars have different shaft stiffness. Medium oar-shafts are referred to as "M" oars and oar-shafts denoted as "ES" are Extra-Soft. The circumferences of Oar M and Oar ES both taper from 0.111 
$\mathrm{m}$ at the sleeves to $0.108 \mathrm{~m}$ at the blades. The shafts have a so-called "skinny" construction and "Fat2" blades were used (Concept2 Inc., Vermont, United

States). Oar M and Oar ES have masses of 1.4 and $1.3 \mathrm{~kg}$, respectively. The two sets of oars were analyzed with three different lengths, for a total of six configurations (Table 8). The outboard length ranged from 1.79 to $1.83 \mathrm{~m}$ and the inboard length was fixed at $0.87 \mathrm{~m}$. All length measurements had an engineering tolerance of $\pm 9 \times 10^{-5} \mathrm{~m}$ (Lufkin, Texas, USA).

Table 8. The six oar configurations that were tested. Each configuration is designated by a code that indicates the stiffness (M or ES) and total length of the oar. The total length of the oar varied by changing the outboard length.

\begin{tabular}{llcc}
\hline Code & Stiffness & Total Length $(\mathrm{m})$ & Outboard Length $(\mathrm{m})$ \\
\hline M2.66 & Medium & 2.66 & 1.79 \\
M2.68 & Medium & 2.68 & 1.81 \\
M2.70 & Medium & 2.70 & 1.83 \\
ES2.66 & Extra-Soft & 2.66 & 1.79 \\
ES2.68 & Extra-Soft & 2.68 & 1.81 \\
ES2.70 & Extra-Soft & 2.70 & 1.83 \\
\hline
\end{tabular}

\subsubsection{Experiment}

Each rower performed a self-directed warm up. The rowers were tested in single sculling boats instrumented with the PL system. Starting from a zero boat velocity relative to the water, the rowers used approximately $100 \mathrm{~m}$ to accelerate to their individual race pace, subsequently rowing an additional $200 \mathrm{~m}$ at a constant race pace for data collection. Race pace refers to the individualized stroke rate that a rower maintains for the majority of their $2000 \mathrm{~m}$ competitive races. Each rower completed six trials, and each trial was used to test a different oar configuration. Table 9 shows the mean stroke rates during each trial for each rower. The maximum difference in stroke rate between the six trials was approximately 2.3 
$\%$. The rowers had $12 \pm 3$ minutes to rest between trials. The experiment was single-blinded whereby the configurations of the oars were unknown to the rowers. The six configurations were tested in a different order for each rower, as shown in Table 10.

Table 9. The mean stroke rates (strokes/min) during each trial for each rower; the uncertainties are SD. The experiment started with trial 1 and ended with trial

6 . The mean $\bar{x}$ stroke rate for each rower across all six trials is also provided.

\begin{tabular}{ccccc}
\hline Trial & Rower 1 & Rower 2 & Rower 3 & Rower 4 \\
\hline 1 & $31.2 \pm 0.5$ & $30.4 \pm 0.4$ & $31.5 \pm 0.5$ & $33.4 \pm 0.5$ \\
2 & $31.1 \pm 0.5$ & $30.5 \pm 0.4$ & $31.4 \pm 0.5$ & $33.7 \pm 0.5$ \\
3 & $31.4 \pm 0.4$ & $30.7 \pm 0.3$ & $31.0 \pm 0.4$ & $33.5 \pm 0.6$ \\
4 & $31.3 \pm 0.4$ & $30.8 \pm 0.4$ & $31.2 \pm 0.5$ & $33.6 \pm 0.5$ \\
5 & $31.3 \pm 0.5$ & $30.8 \pm 0.5$ & $31.7 \pm 0.3$ & $33.1 \pm 0.5$ \\
6 & $31.0 \pm 0.5$ & $30.7 \pm 0.4$ & $31.0 \pm 0.4$ & $33.6 \pm 0.4$ \\
$\bar{x}$ & $31.2 \pm 0.1$ & $30.7 \pm 0.2$ & $31.3 \pm 0.3$ & $33.5 \pm 0.2$ \\
\hline
\end{tabular}

Table 10. Testing the six oar configurations in a different order for each rower.

\begin{tabular}{ccccc}
\hline Trial & Rower 1 & Rower 2 & Rower 3 & Rower 4 \\
\hline 1 & M2.70 & ES2.68 & ES2.66 & M2.68 \\
2 & ES2.68 & M2.70 & M2.68 & ES2.66 \\
3 & M2.68 & ES2.66 & ES2.70 & M2.66 \\
4 & ES2.66 & M2.68 & M2.66 & ES2.70 \\
5 & M2.66 & ES2.70 & M2.70 & ES2.68 \\
6 & ES2.70 & M2.66 & ES2.68 & M2.70 \\
\hline
\end{tabular}




\subsubsection{Instrumentation}

An anemometer (Krestrel 2000 Pocket Wind Meter, Nielsen-Kellerman, United States) was used to measure the average wind velocity along the testing course during each trial. The anemometer has an engineering tolerance of $\pm 3 \%$ of the wind measurement [18]. The experiments were conducted on the conservative condition that the measured wind velocities be less than $2.5 \mathrm{~m} / \mathrm{s}$ (Table 11). There was a Pearson product-moment correlation coefficient $r$ of .24 between the measured wind velocities and $200 \mathrm{~m}$ performance times. The Pearson $r$ quantifies the strength of a linear association between two variables, and can range between -1 and +1 [19].

Table 11. Average wind velocity $(\mathrm{m} / \mathrm{s})$ measured along the testing course during trials 1-6 for each rower.

\begin{tabular}{ccccc}
\hline Trial & Rower 1 & Rower 2 & Rower 3 & Rower 4 \\
\hline 1 & 0.3 & 0.3 & 0.8 & 0.6 \\
2 & 0.5 & 0.5 & 1.0 & 1.0 \\
3 & 1.1 & 0.8 & 0.4 & 0.4 \\
4 & 0.8 & 1.0 & 0.0 & 0.0 \\
5 & 0.9 & 0.9 & 0.4 & 0.4 \\
6 & 0.9 & 0.7 & 0.8 & 0.8 \\
\hline
\end{tabular}

Accelerometers (Peach Innovations Ltd., Cambridge, United Kingdom) were mounted to the inside of the rowing shells and were orientated in the sternbow axis; the accelerometers are calibrated to measure proper accelerations in the $x$-direction. In addition, 200 m performance times were measured using manually operated digital chronographs (Interval 2000, Nielsen-Kellerman, United States). According to the National Institute of Standards and Technology, the measurement error associated with human reaction time using manually operated chronographs is approximately $\pm 0.1 \mathrm{~s}[20]$. 
Oarlock biomechanics were measured using the PL Rowing Instrumentation System (Peach Innovations Ltd., Cambridge, United Kingdom). To recall, the PL system features replacement oarlocks that measure the angular displacement of the swivel about pin via two Hall effect sensors and an 8-axial pole ring magnet [21]. The angle measurements have an engineering tolerance of $\pm 0.5^{\circ}$ [22]. The PL oarlocks are also instrumented with load cells, which measure the forces applied to the PL swivels in the $x$-direction [21]. The results in Chapter 3 show that the PL force measurements were consistent over multiple days of testing, but were slightly less than the values of the known applied forces. Accordingly, calibration factors for each PL oarlock were established to correct for the discrepancies in the force measurements.

Each boat had the rower's customized foot-stretcher, seat and oarlock settings. These settings did not change while the boats were instrumented with the PL oarlocks and accelerometers. However, the distance between the starboard and port pins (i.e., the span) was set to $1.58 \mathrm{~m}$ for all boats. The oarlock force and angle measurements were zeroed using a protocol outlined by the PL manufacturers [22]. Data-loggers were mounted to the inside of the rowing shells, and were connected to the PL oarlocks and accelerometers. The loggers store the data measured during on-water rowing. The loggers were removed from the shells post-testing, and the data were downloaded to PC software for analysis.

\subsubsection{Data Analysis and Signal Processing}

Data were analyzed and processed in MATLAB 2013a (The MathWorks Inc., Massachusetts, USA). The calibration factors outlined in Chapter 3 were applied to the force data from each PL oarlock. To supress unwanted features of the signal, data were smoothed using a moving average recursive filter with a window-based finite impulse response design. The results presented below are arithmetic means over 20 strokes with uncertainties given by SD. The fitted curves in the figures are smoothing splines. Since the rower does not apply 
propulsive forces to the oar during the recovery [23], only the results from the drive phase are presented. Note that the start of the drive is at the catch position.

\subsection{Results}

\subsubsection{Stiffness}

Figures 17-20 show the boat acceleration as a percentage of the drive for ES2.70 and M2.70 for rowers 1-4. Each rower had a distinctive acceleration curve. The relative differences in the curves between the two oar configurations, for a given rower, were primarily of interest. There were very small observable differences in the acceleration curves between Oar M and Oar ES. These differences were presumably on the same order of magnitude as the rower's inter-stroke inconsistencies (i.e., shown in the scatter of the data). The relative differences in the acceleration curves between the two oar configurations were consistent across the all four rowers. Similar results were observed at oar lengths of 2.66 and $2.68 \mathrm{~m}$. 


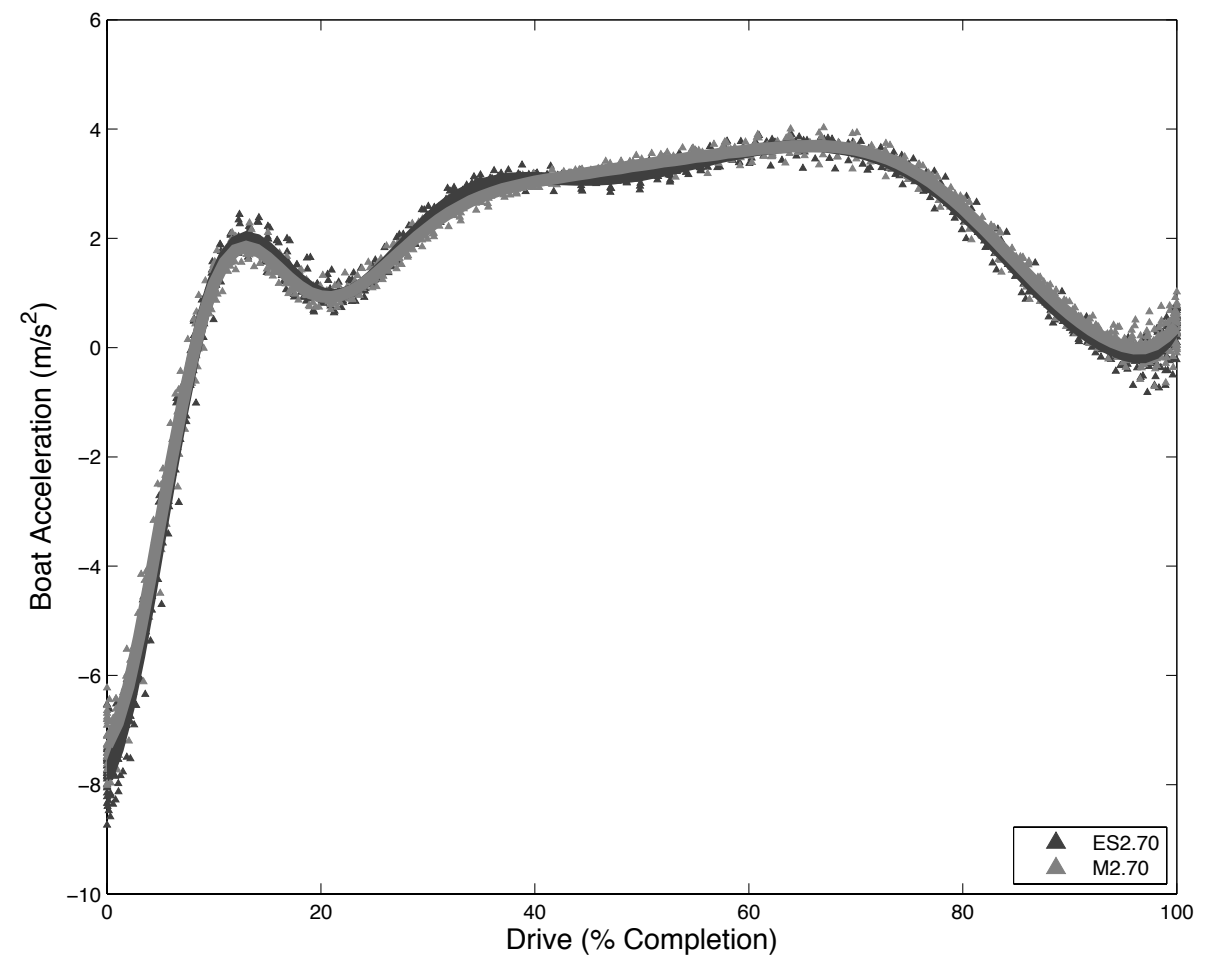

Figure 17. Boat acceleration as a percentage of the drive between ES2.70 and M2.70 for rower 1 . The fits to the data are smoothing splines.

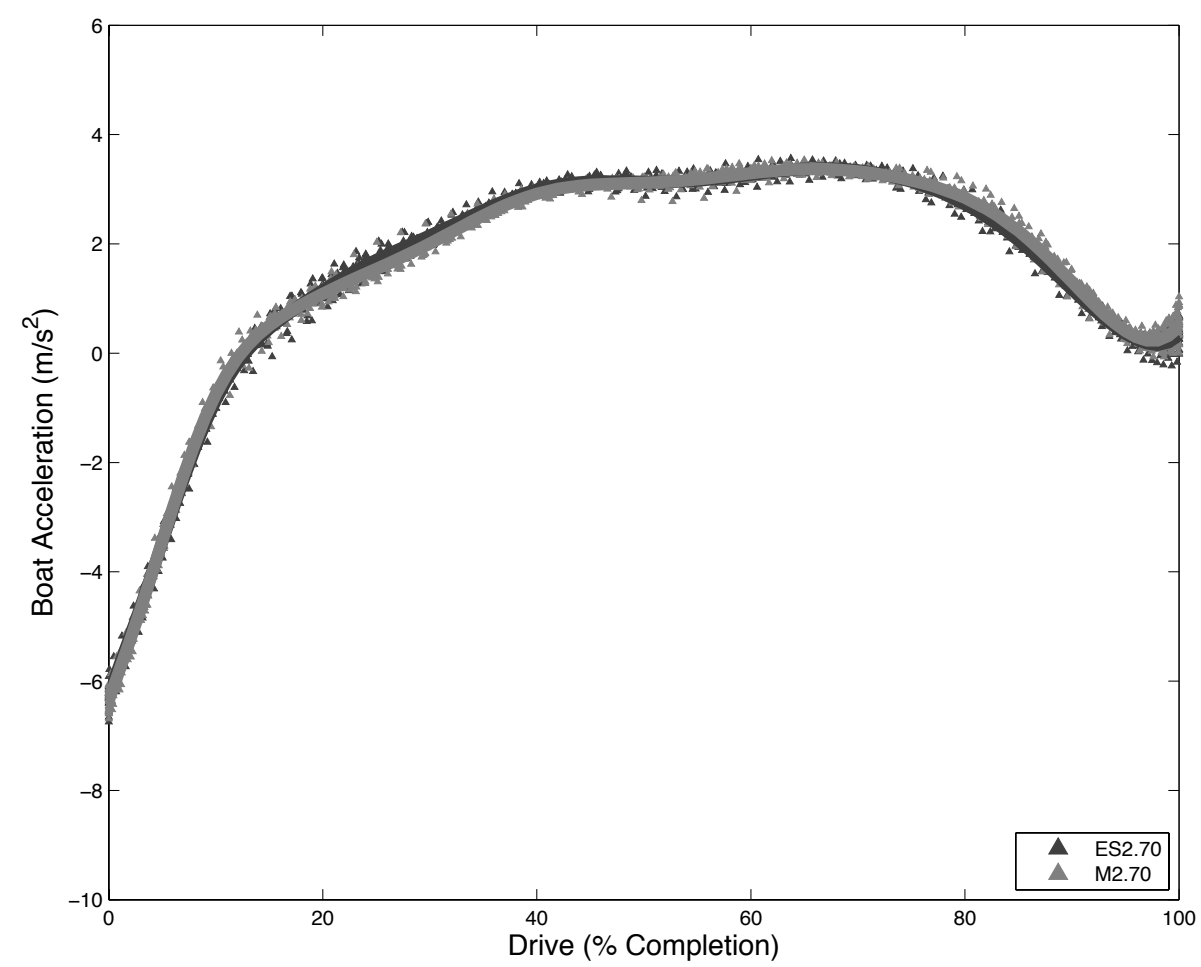

Figure 18. Boat acceleration as a percentage of the drive between ES2.70 and M2.70 for rower 2. The fits to the data are smoothing splines. 


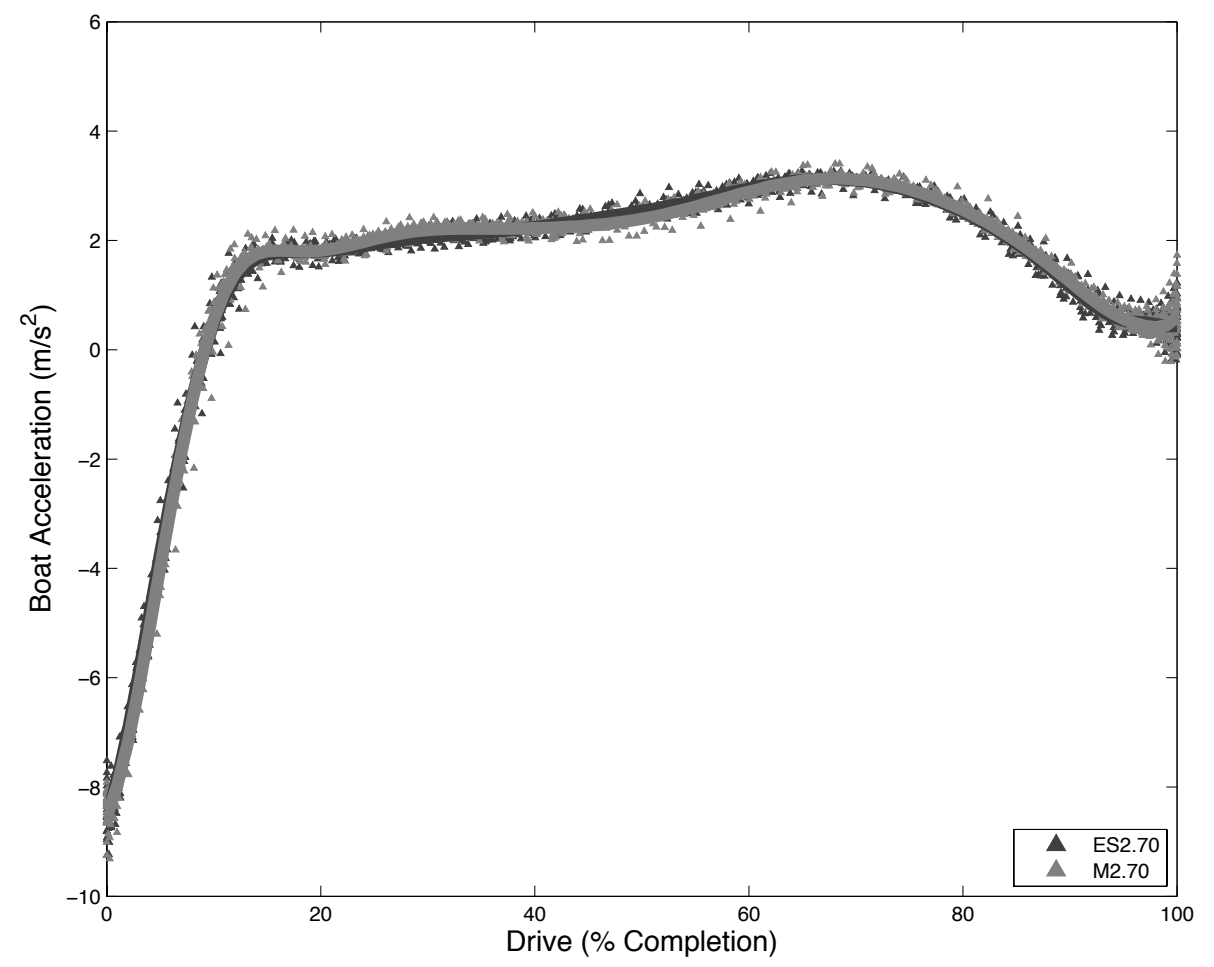

Figure 19. Boat acceleration as a percentage of the drive between ES2.70 and M2.70 for rower 3. The fits to the data are smoothing splines.

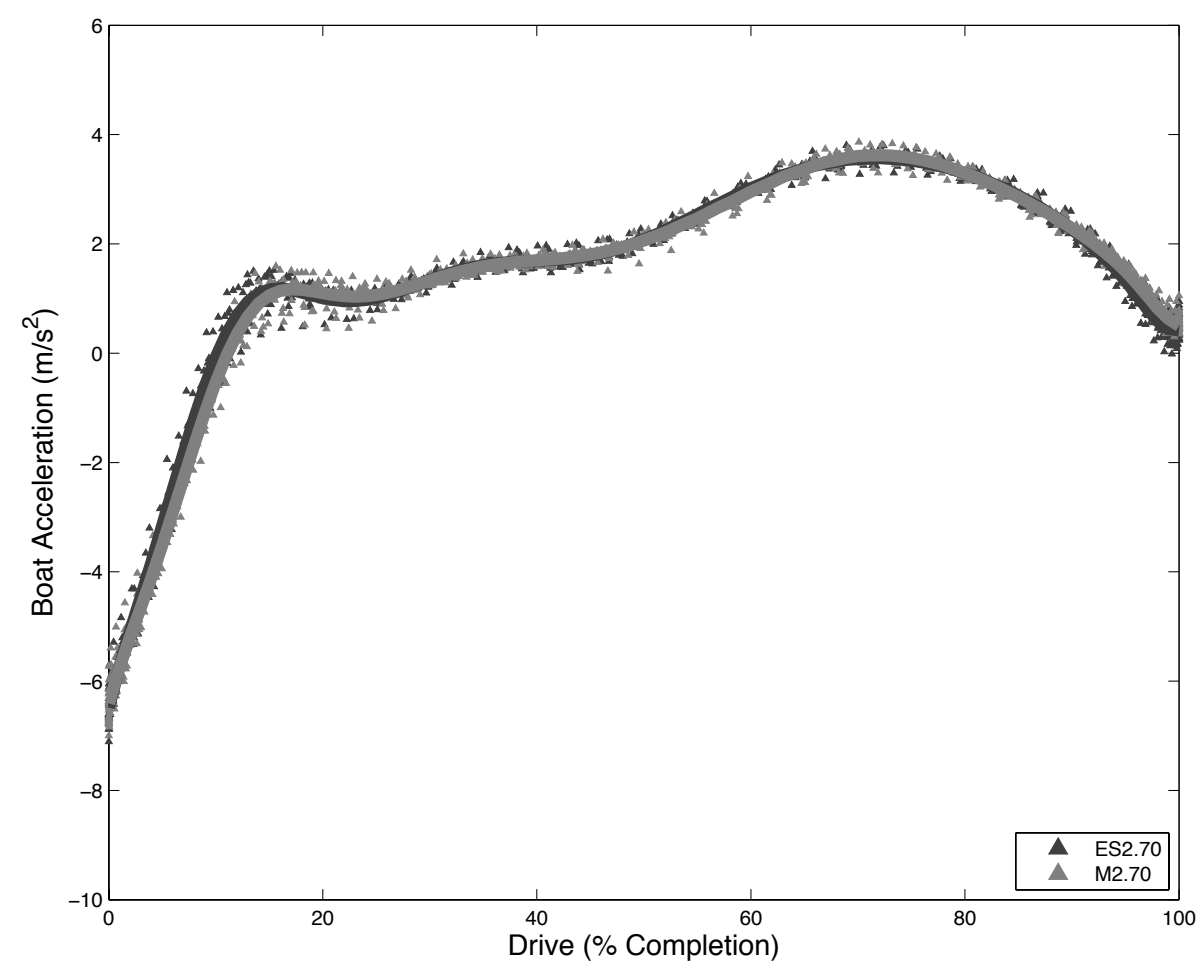

Figure 20. Boat acceleration as a percentage of the drive between ES2.70 and M2.70 for rower 4. The fits to the data are smoothing splines. 
Figures 21-24 show the port oarlock force as a function of the oarlock angle for ES2.70 and M2.70 for rowers 1-4. As expected, each rower had a distinctive force curve. There were slight differences in the force curves between ES2.70 and M2.70, particularly around the area of maximum force $F_{\max }$. However, these differences were presumably less than the rower's inter-stroke inconsistencies. The following descriptive statistics include both port and starboard data. Oar ES had a $2.5 \% \pm 3.6$ pp higher $\mathrm{F}_{\max }$ than Oar $\mathrm{M}$ at an oar length of $2.70 \mathrm{~m}$; the inter-stroke inconsistencies in $\mathrm{F}_{\max }$ were $3.3 \% \pm 0.9 \mathrm{pp}$. This trend in the means was not systematic across oar lengths. Oar M had a 2.3 $\% \pm 4.2$ pp higher $F_{\max }$ than Oar ES at $2.68 \mathrm{~m}$, but a $0.5 \% \pm 3.3$ pp lower $F_{\max }$ than Oar ES at $2.66 \mathrm{~m}$; the inter-stroke inconsistencies in $\mathrm{F}_{\max }$ were $3.5 \% \pm 0.8$ pp at $2.68 \mathrm{~m}$ and $3.6 \% \pm 1.5 \mathrm{pp}$ at $2.66 \mathrm{~m}$. Table 12 shows the $F_{\max }$ for each rower for each configuration.

The percentage of the drive from the catch to the point of maximum force $\mathrm{F}_{\max \%}$ is also of interest. Oar $\mathrm{M}$ reached $\mathrm{F}_{\max } 2.1 \% \pm 7.2 \mathrm{pp}$ faster than Oar ES at an oar length of $2.70 \mathrm{~m}$. However, the $F_{\max \%}$ varied by $9.4 \% \pm 6.0 \mathrm{pp}$ between strokes. This trend in the means was consistent across oar lengths whereby Oar $\mathrm{M}$ reached $\mathrm{F}_{\max }$ quicker than Oar ES by $1.3 \% \pm 5.5 \mathrm{pp}$ at $2.68 \mathrm{~m}$ and $3.3 \% \pm 3.9$ $\mathrm{pp}$ at $2.66 \mathrm{~m}$. Once again, the inter-stroke inconsistencies in the $\mathrm{F}_{\max \%}$ were greater than the differences between Oar M and Oar ES. While the integral of the oarlock force with respect its angular displacement, including the inboard length, is the amount of work done, the integral of the applied force over time is the impulse on the oarlock. Oar ES had a $3.4 \% \pm 4.0$ pp larger impulse than Oar M at an oar length of $2.70 \mathrm{~m}$. However, the impulse varied by $3.2 \% \pm 1.0 \mathrm{pp}$ between strokes. This trend in the means was not systematic across oar lengths. Oar M had a $1.8 \% \pm 6.5 \mathrm{pp}$ larger impulse than Oar ES at $2.68 \mathrm{~m}$, but a $0.2 \% \pm$ $11.7 \mathrm{pp}$ smaller impulse than Oar ES at $2.66 \mathrm{~m}$; the inter-stroke inconsistencies in the impulse were $3.3 \% \pm 0.9 \mathrm{pp}$ at $2.68 \mathrm{~m}$ and $3.2 \% \pm 1.3 \mathrm{pp}$ at $2.66 \mathrm{~m}$. The $F_{\max \%}$ and the impulse for each rower for each configuration are shown in Table 12. 
Table 12. The maximum force $(\mathrm{N}), \%$ of the drive to maximum force, and impulse (Ns) measured on port and starboard oarlocks for each oar configuration for all four rowers. The results are given by the mean \pm SD over 20 strokes.

\begin{tabular}{|c|c|c|c|c|c|c|}
\hline $\begin{array}{c}\text { Test } \\
\text { Description }\end{array}$ & Port $F_{\max }$ & $\begin{array}{c}\text { Starboard } \\
F_{\max }\end{array}$ & Port $F_{\max \%}$ & $\begin{array}{c}\text { Starboard } \\
\mathrm{F}_{\max \%}\end{array}$ & Port Impulse & $\begin{array}{l}\text { Starboard } \\
\text { Impulse }\end{array}$ \\
\hline R1 ES2.66 & $358.7 \pm 11.6$ & $322.3 \pm 27.7$ & $35.7 \pm 1.1$ & $37.6 \pm 2.2$ & $174.2 \pm 4.2$ & $168.1 \pm 9.9$ \\
\hline R1 ES2.68 & $349.3 \pm 13.9$ & $303.5 \pm 11.7$ & $38.3 \pm 2.4$ & $35.8 \pm 3.1$ & $168.8 \pm 5.8$ & $162.0 \pm 5.8$ \\
\hline R1 ES2.70 & $373.7 \pm 9.9$ & $345.9 \pm 15.5$ & $35.9 \pm 1.3$ & $36.2 \pm 1.7$ & $184.8 \pm 6.0$ & $184.2 \pm 7.3$ \\
\hline R1 M2.66 & $369.7 \pm 11.1$ & $321.0 \pm 14.0$ & $36.0 \pm 1.9$ & $33.9 \pm 3.2$ & $184.0 \pm 6.3$ & $171.9 \pm 8.0$ \\
\hline R1 M2.68 & $367.4 \pm 8.2$ & $337.0 \pm 16.8$ & $35.3 \pm 1.2$ & $34.0 \pm 1.8$ & $179.2 \pm 3.9$ & $174.5 \pm 6.4$ \\
\hline R1 M2.70 & $368.3 \pm 8.0$ & $317.5 \pm 16.1$ & $36.3 \pm 1.4$ & $35.4 \pm 2.2$ & $181.7 \pm 4.3$ & $170.3 \pm 8.8$ \\
\hline R2 ES.266 & $317.7 \pm 7.1$ & $268.4 \pm 5.9$ & $39.0 \pm 1.5$ & $37.4 \pm 1.9$ & $168.9 \pm 2.4$ & $136.3 \pm 3.2$ \\
\hline R2 ES2.68 & $313.3 \pm 8.7$ & $230.7 \pm 7.3$ & $40.2 \pm 1.3$ & $37.5 \pm 1.7$ & $165.1 \pm 4.6$ & $115.9 \pm 3.7$ \\
\hline R2 ES2.70 & $318.8 \pm 7.5$ & $247.8 \pm 7.9$ & $37.3 \pm 1.0$ & $35.1 \pm 1.3$ & $173.4 \pm 3.3$ & $129.8 \pm 4.6$ \\
\hline R2 M2.66 & $319.2 \pm 8.6$ & $279.9 \pm 8.9$ & $38.8 \pm 2.2$ & $35.0 \pm 2.6$ & $167.6 \pm 3.1$ & $137.7 \pm 4.3$ \\
\hline R2 M2.68 & $309.3 \pm 6.9$ & $246.6 \pm 10.6$ & $38.0 \pm 1.0$ & $36.3 \pm 1.7$ & $160.8 \pm 2.8$ & $127.5 \pm 4.5$ \\
\hline R2 M2.70 & $312.2 \pm 6.4$ & $245.0 \pm 11.3$ & $39.2 \pm 1.6$ & $37.2 \pm 2.2$ & $169.9 \pm 2.1$ & $125.0 \pm 5.0$ \\
\hline R3 ES2.66 & $312.1 \pm 10.3$ & $311.6 \pm 11.6$ & $40.0 \pm 1.9$ & $36.9 \pm 2.4$ & $173.7 \pm 6.7$ & $179.2 \pm 4.1$ \\
\hline R3 ES2.68 & $305.7 \pm 10.9$ & $292.3 \pm 11.1$ & $37.3 \pm 3.4$ & $35.0 \pm 3.4$ & $182.4 \pm 2.8$ & $167.7 \pm 5.0$ \\
\hline R3 ES2.70 & $285.0 \pm 9.9$ & $304.9 \pm 9.9$ & $33.8 \pm 3.7$ & $29.1 \pm 3.9$ & $167.5 \pm 6.9$ & $178.0 \pm 3.7$ \\
\hline R3 M2.66 & $297.8 \pm 8.4$ & $296.6 \pm 10.7$ & $38.0 \pm 3.1$ & $34.9 \pm 3.5$ & $170.8 \pm 3.2$ & $169.6 \pm 3.6$ \\
\hline R3 M2.68 & $290.4 \pm 11.7$ & $311.2 \pm 9.5$ & $37.4 \pm 3.7$ & $34.2 \pm 4.3$ & $167.1 \pm 6.3$ & $177.2 \pm 5.0$ \\
\hline R3 M2.70 & $296.2 \pm 10.5$ & $299.6 \pm 8.9$ & $34.8 \pm 3.0$ & $31.0 \pm 3.9$ & $175.2 \pm 4.0$ & $174.3 \pm 4.2$ \\
\hline R4 ES2.66 & $303.1 \pm 11.5$ & $330.7 \pm 10.8$ & $46.1 \pm 6.8$ & $46.5 \pm 8.7$ & $164.7 \pm 7.7$ & $176.0 \pm 7.1$ \\
\hline R4 ES2.68 & $301.8 \pm 9.1$ & $323.5 \pm 11.7$ & $45.0 \pm 6.8$ & $44.2 \pm 8.4$ & $168.1 \pm 7.1$ & $176.0 \pm 8.6$ \\
\hline R4 ES2.70 & $315.1 \pm 10.0$ & $333.3 \pm 9.6$ & $44.2 \pm 6.2$ & $43.0 \pm 7.8$ & $176.5 \pm 5.3$ & $176.7 \pm 6.2$ \\
\hline R4 M2.66 & $301.9 \pm 8.6$ & $323.9 \pm 13.3$ & $44.0 \pm 4.7$ & $43.7 \pm 6.2$ & $160.1 \pm 5.3$ & $168.5 \pm 6.5$ \\
\hline R4 M2.68 & $306.4 \pm 8.4$ & $323.4 \pm 12.7$ & $46.8 \pm 5.6$ & $48.0 \pm 7.0$ & $166.6 \pm 5.7$ & $169.7 \pm 8.1$ \\
\hline $\mathrm{R} 4 \mathrm{M} 2.70$ & $301.1 \pm 10.7$ & $320.5 \pm 9.1$ & $47.0 \pm 8.2$ & $47.3 \pm 9.8$ & $163.7 \pm 6.7$ & $167.3 \pm 6.4$ \\
\hline
\end{tabular}


There was no consistent trend across all four rowers in $200 \mathrm{~m}$ performance times between Oar M and Oar ES. Table 13 shows the $200 \mathrm{~m}$ performance times for each rower for each trial. On average, rowers 1 and 2 were $0.8 \% \pm 0.3 \mathrm{pp}$ and $0.5 \% \pm 0.3 \mathrm{pp}$ faster with Oar M than Oar ES. In contrast, rowers 3 and 4 were $1.2 \% \pm 2.6$ pp and $1.2 \% \pm 0.1$ pp faster with Oar ES than Oar M. Subjective feedback from the rowers indicates they could "feel" a difference between Oar M and Oar ES. However, they were unable to correctly identify the stiffness classification of each oar configuration.

Table 13. $200 \mathrm{~m}$ performance time (s) for each rower for each trial. The mean $\overline{\boldsymbol{x}}$ performance time for each rower across all six trials is also provided.

\begin{tabular}{ccccc}
\hline Trial & Rower 1 & Rower 2 & Rower 3 & Rower 4 \\
\hline 1 & 47.8 & 47.7 & 45.9 & 47.4 \\
2 & 48.7 & 48.3 & 46.4 & 46.6 \\
3 & 48.4 & 48.8 & 46.7 & 47.1 \\
4 & 49.2 & 48.4 & 47.5 & 46.6 \\
5 & 49.2 & 48.7 & 47.6 & 47.4 \\
6 & 48.3 & 48.7 & 47.2 & 47.2 \\
$\bar{x}$ & $48.6 \pm 0.5$ & $48.4 \pm 0.4$ & $46.9 \pm 0.7$ & $47.1 \pm 0.4$ \\
\hline
\end{tabular}




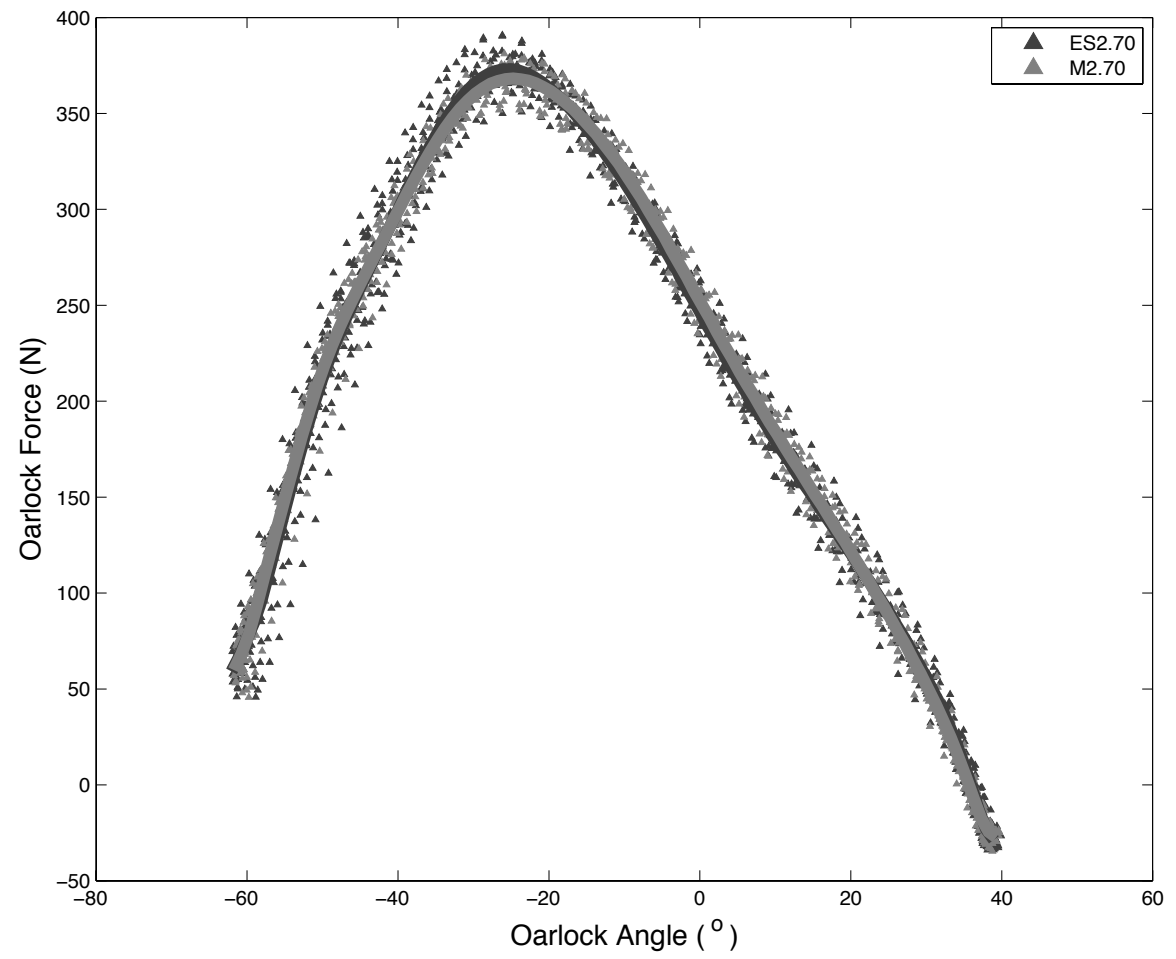

Figure 21. Oarlock force as a function of angle between ES2.70 and M2.70 for rower 1 on port side. The fits are smoothing splines.

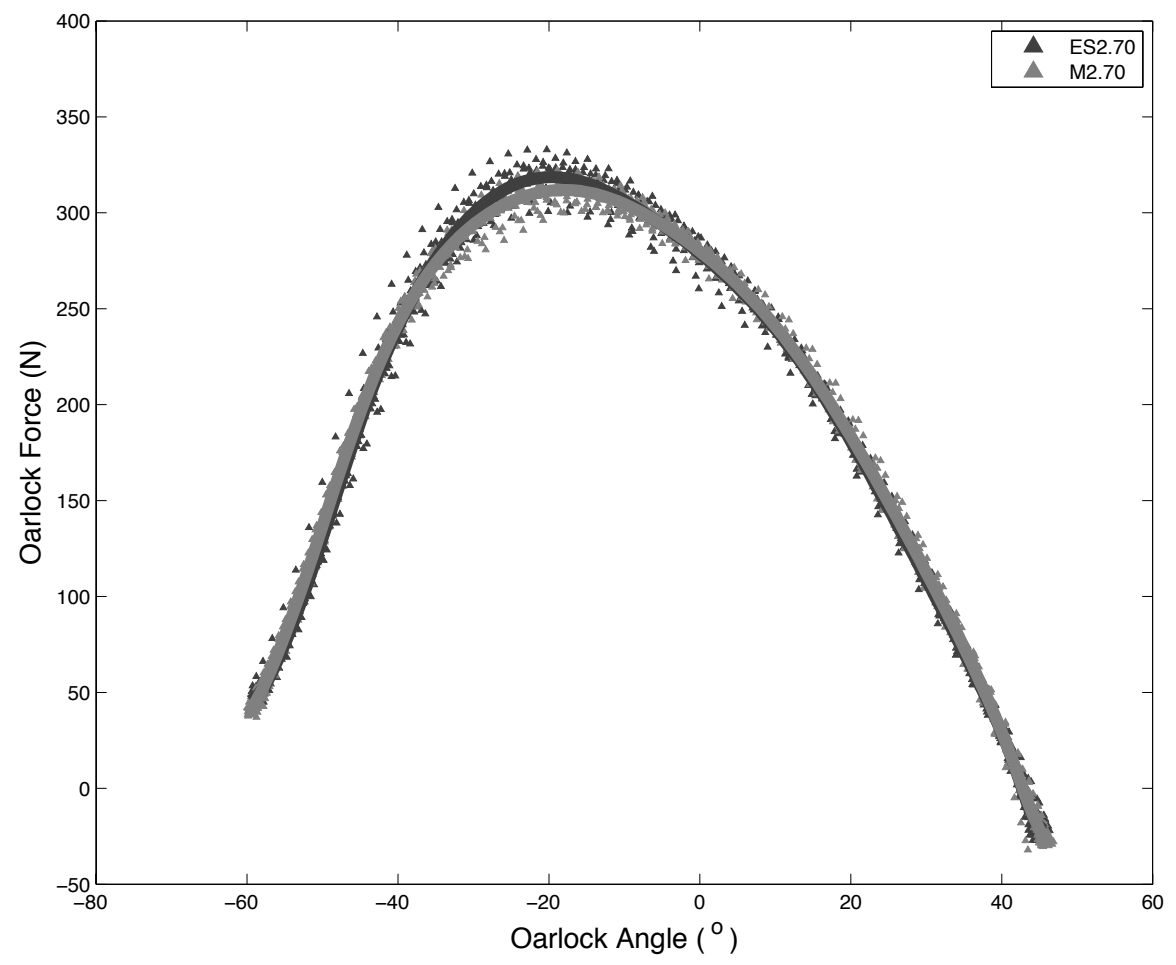

Figure 22. Oarlock force as a function of angle between ES2.70 and M2.70 for rower 2 on port side. The fits are smoothing splines. 


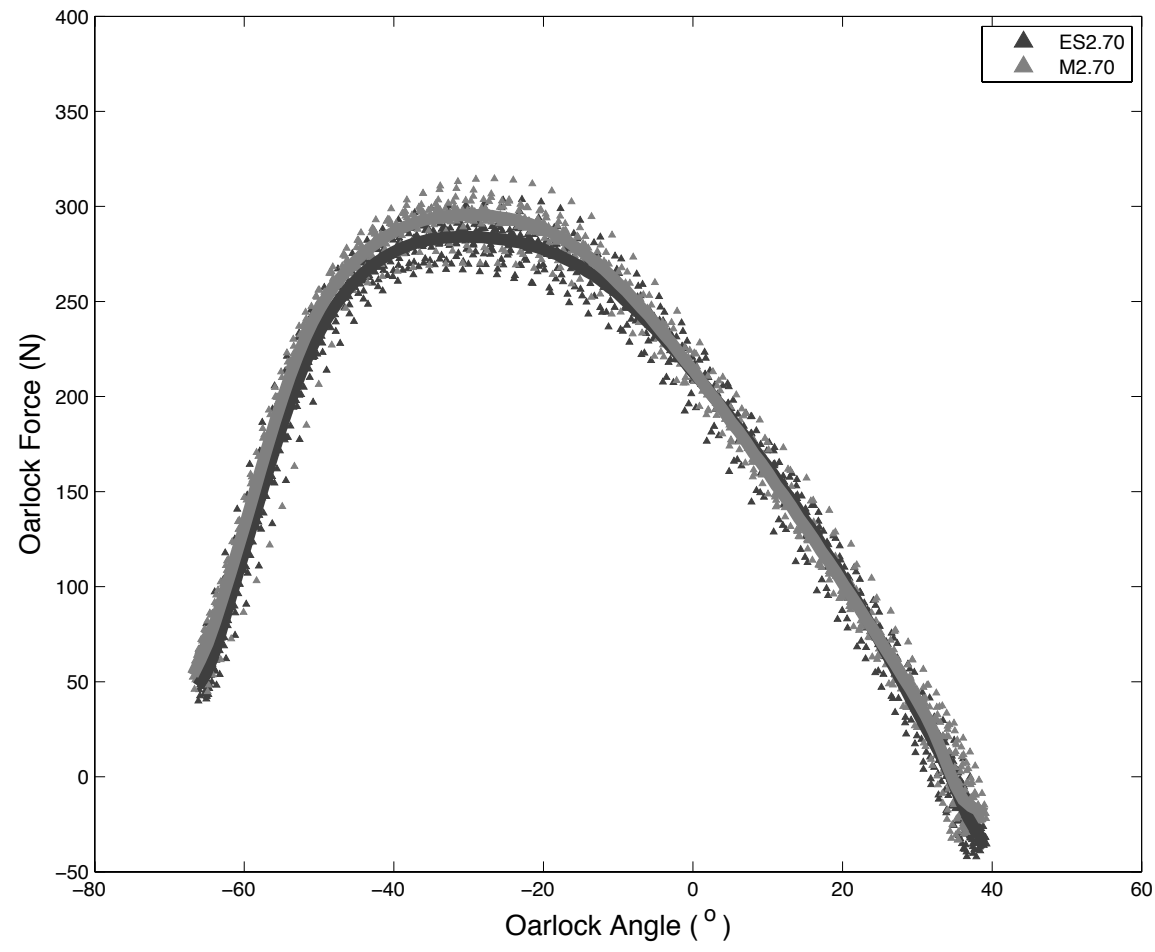

Figure 23. Oarlock force as a function of angle between ES2.70 and M2.70 for rower 3 on port side. The fits are smoothing splines.

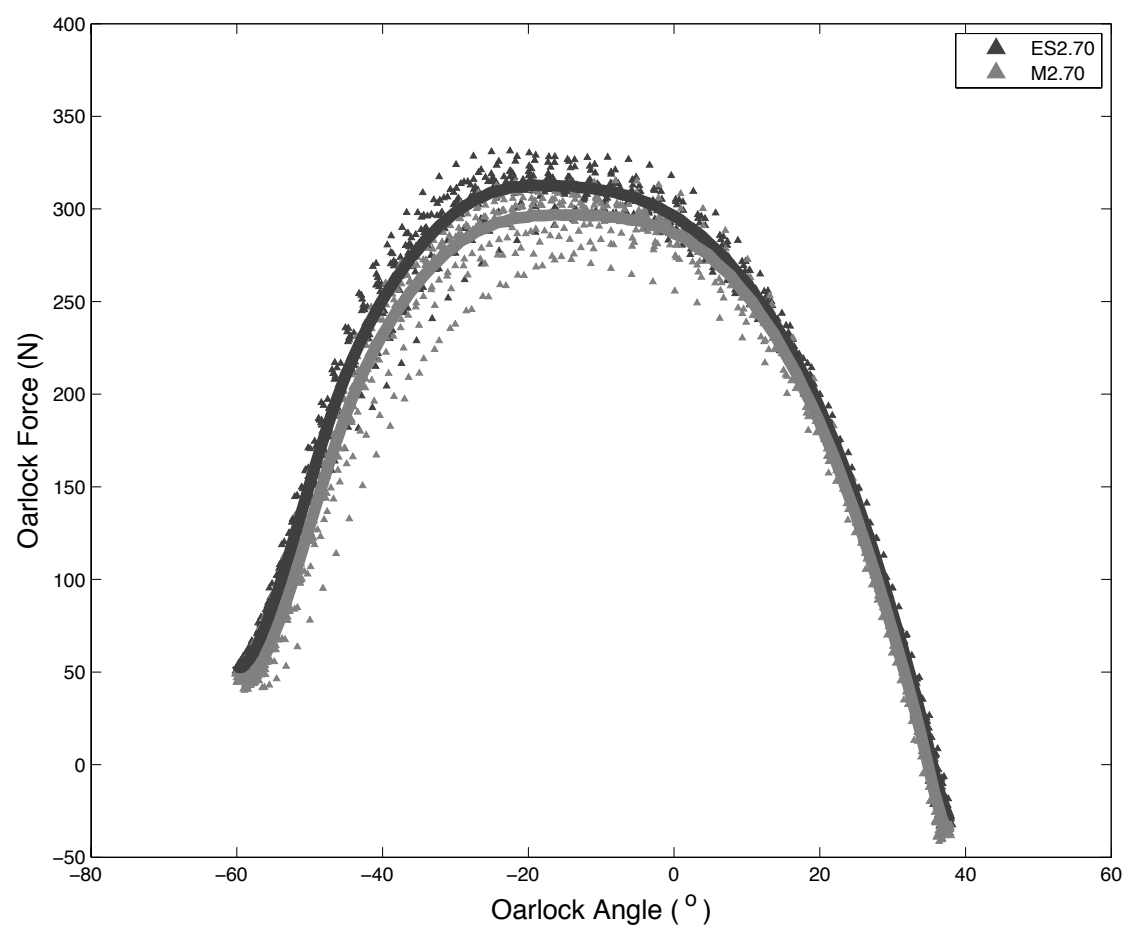

Figure 24. Oarlock force as a function of angle between ES2.70 and M2.70 for rower 4 on port side. The fits are smoothing splines. 


\subsubsection{Length}

Figures 25-28 show the port oarlock force as a function of the oarlock angle for M2.66 and M2.70 for rowers 1-4. The figures show slight differences in the force curves between M2.66 and M2.70, particularly around the area of $F_{\max }$. However, these differences were presumably less than the rower's inter-stroke inconsistencies (i.e., shown in the scatter of the data). The following descriptive statistics include both port and starboard data for Oar $\mathrm{M}$ for all four rowers. There was a weak negative correlation between oar length and $F_{\max }$, with a Pearson $r$ of $-.09 \pm .14$. Oars of $2.66 \mathrm{~m}$ had a $2.4 \% \pm 4.9 \mathrm{pp}$ higher $F_{\max }$ than oars of 2.70 $\mathrm{m}$; although the $\mathrm{F}_{\max }$ varied by $3.4 \% \pm 0.9$ pp between strokes. Similar results were observed for Oar ES.

Oars of $2.66 \mathrm{~m}$ also reached $\mathrm{F}_{\max } 2.4 \% \pm 5.7 \mathrm{pp}$ quicker than oars of 2.70 $\mathrm{m}$; the $\mathrm{F}_{\max \%}$ varied by $9.4 \% \pm 4.8 \mathrm{pp}$ between strokes. There was a strong positive correlation between oar length and $F_{\max }$, with a Pearson $r$ of $.55 \pm .21$. In addition, oars of $2.66 \mathrm{~m}$ had a $0.5 \% \pm 4.2 \mathrm{pp}$ larger impulse than oars of 2.70 $\mathrm{m}$. The inter-stroke inconsistencies in the impulse were greater than the differences between the oars of different length. There was a strong negative correlation $(r=-.43 \pm .67)$ between oar length and impulse. Similar differences in the $F_{\max \%}$ and the impulse between oars of 2.66 and $2.70 \mathrm{~m}$ were observed for Oar ES.

There was a strong negative correlation $(r=-.99 \pm .01$ and $-.52 \pm .62)$ between oar length and $200 \mathrm{~m}$ performance times for rowers 1 and 2 . In contrast, rowers 3 and 4 showed a strong positive correlation $(r=.34 \pm .38$ and $.63 \pm .43)$ between oar length and $200 \mathrm{~m}$ performance times. 


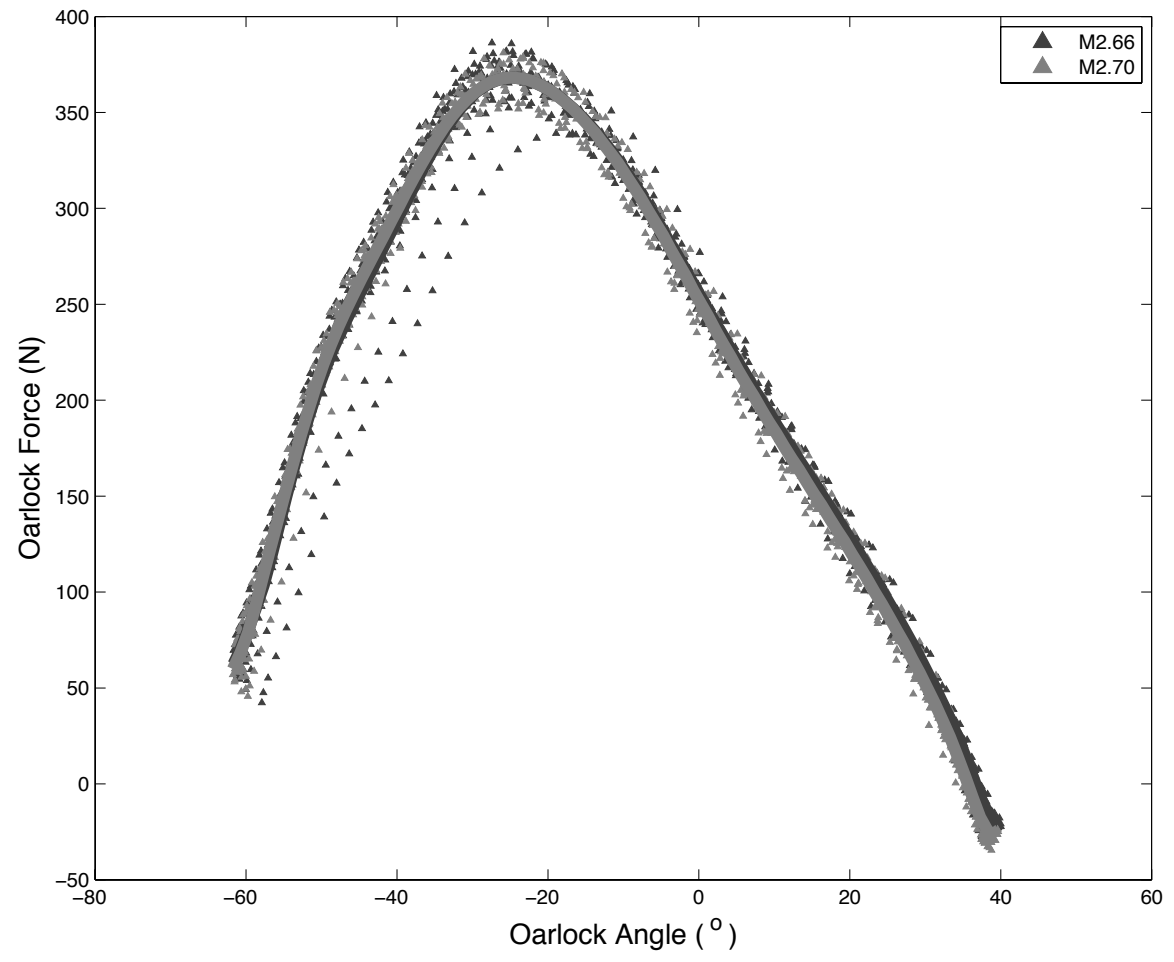

Figure 25. Oarlock force as a function of angle between M2.66 and M2.70 for rower 1 on port side. The fits are smoothing splines.

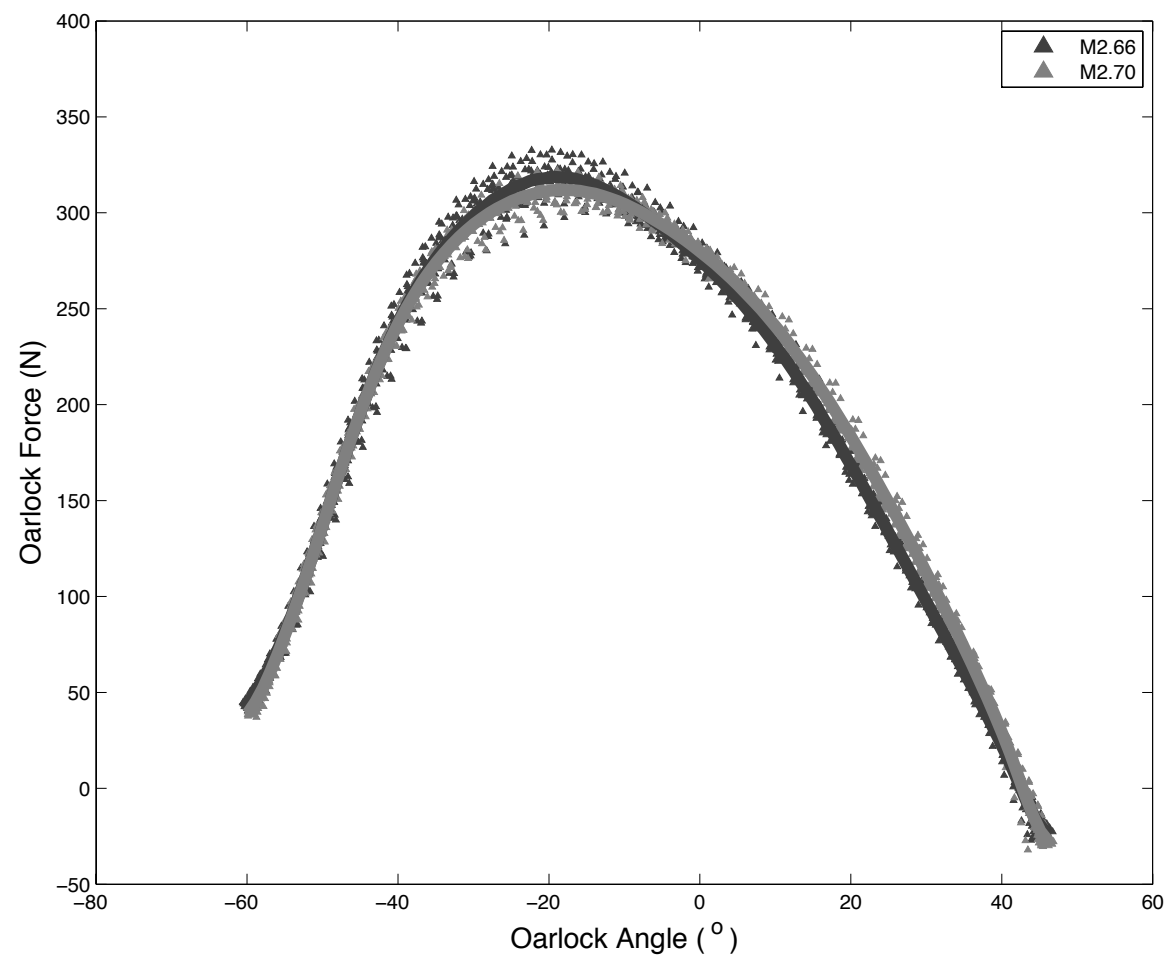

Figure 26. Oarlock force as a function of angle between M2.66 and M2.70 for rower 2 on port side. The fits are smoothing splines. 


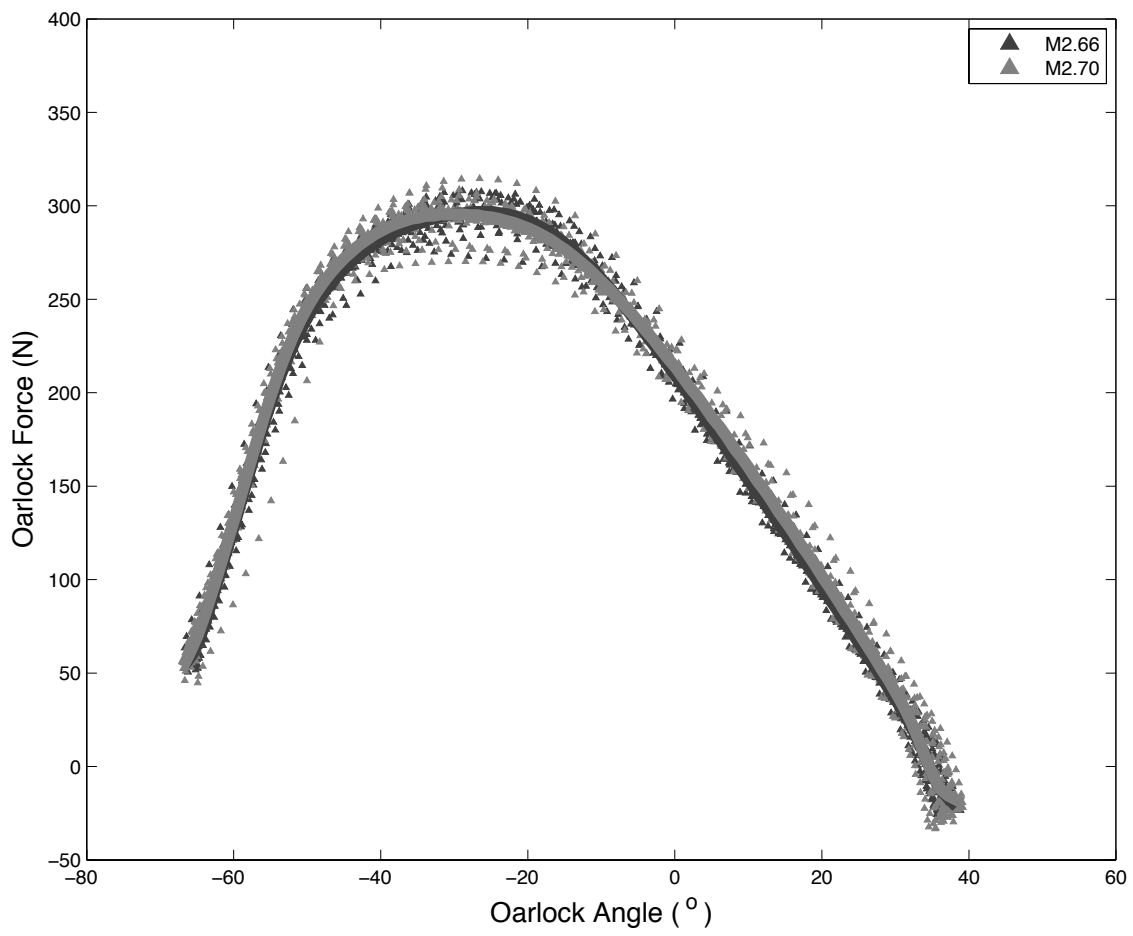

Figure 27. Oarlock force as a function of angle between M2.66 and M2.70 for rower 3 on port side. The fits are smoothing splines.

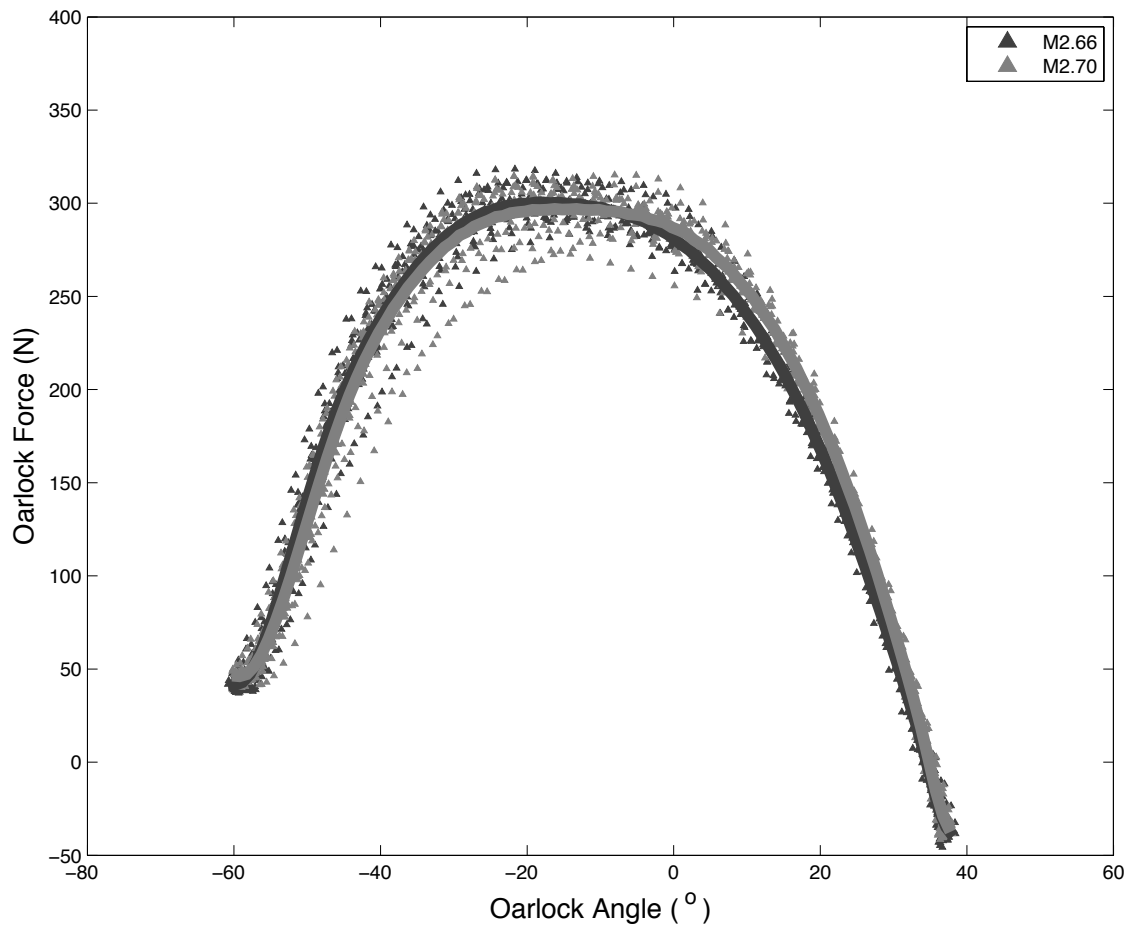

Figure 28. Oarlock force as a function of angle between M2.66 and M2.70 for rower 4 on port side. The fits are smoothing splines. 


\subsection{Discussion}

The purpose of this chapter was to investigate the biomechanics of rowing with oar-shafts of different stiffness and length. Rowers were tested in instrumented single boats over $200 \mathrm{~m}$ at race pace; the mean inter-rower stroke rate was 31.6 \pm 1.1 strokes $/ \mathrm{min}$. In comparison, the mean stroke rate of all medalists in the women's single sculling final at the 2000 Olympic games was 33.5 strokes $/ \mathrm{min}$ [24]. As such, the results presented in this chapter originate from biomechanical measurements taken at rowing intensities that parallel those in elite competition. The rower's stroke rates and the wind velocities were both relatively consistent between trials, and therefore were considered only minor influences in the differences in performance between trials. To minimize physical fatigue, the rowers had $12 \pm 3$ minutes to rest between trials.

The effect of oar-shaft stiffness on proper boat acceleration was examined. There were small differences in the boat accelerations between Medium and Extra-Soft oar-shafts. The differences in acceleration between the oars of different stiffness were presumably on the same order of magnitude as the rower's inter-stroke inconstancies. These findings contradict the notion that less stiff oar-shafts increase boat acceleration towards the end of the drive [12] via high rates of inverse deflection. It is projected that the water provides a damping effect on the blade's movement, and thus prevents the oar-shafts from inversely deflecting at such high rates. Future research should consider instrumenting oar-shafts of different stiffness with technology capable of measuring the rate and magnitude of deflection and inverse deflection during the drive.

High rates of force development at the beginning of the drive are advantageous to rowing performance [25-28]. Less stiff oar-shafts presumably deflect more than stiffer oars during the drive, and therefore provide less resistance and decrease the amount of force the rower can exert onto the handles. This could explain why Extra-Soft oar-shafts showed slightly lower rates 
of force development to maximum force than Medium oars. However, the differences in the rates to maximum force between the oars of different stiffness were less than the rower's inter-stroke inconsistencies.

The impulse on the oarlock was integrated via oarlock force as a function of drive time. There were small differences in the impulse between Medium and Extra-Soft oar-shafts. However, the differences in impulse were less than the rower's inter-stroke inconsistencies. Previous research [29] indicates that "any increase in momentum, and therefore increase in boat velocity, will be determined by the size of the impulse on the oarlock". Accordingly, the interrower inconsistencies in the differences in the mean impulse between Medium and Extra-Soft oar-shafts could explain the inter-rower inconsistencies in the mean boat velocities (i.e., derived from the performance times over $200 \mathrm{~m}$ ) between the oars of different stiffness. However, the exact correlation between the impulse on the oarlock and the mean boat velocity was not analyzed in this work.

Using lever-theory, Nolte [13] projected that shorter oars are more effective in rowing. In this work, oars of $2.66 \mathrm{~m}$ showed slightly higher maximum forces and larger impulses on the oarlocks than oars of $2.70 \mathrm{~m}$. In addition, oars of $2.66 \mathrm{~m}$ showed slightly faster rates of force development to maximum force than oars of $2.70 \mathrm{~m}$. However, the rower's inter-stroke inconsistencies in these biomechanical parameters were greater than the differences between oars of 2.66 and $2.70 \mathrm{~m}$. As such, changing oar length by approximately $1.5 \%$ (i.e., between 2.66 and $2.70 \mathrm{~m}$ ) did not drastically affect rowing biomechanics. These findings are in agreement with an earlier pilot study [17], which tested two single sculling rowers with oars of different lengths (i.e., 2.62, 2.67, and $2.72 \mathrm{~m}$ ). The blade design and inboard length (i.e., $0.87 \mathrm{~m}$ ) were both constant across the different oar configurations. The authors reported, "some variation in force application is noticeable, but the majority of variables are quite similar in these very different rigging settings". Therefore, changing oar length by approximately $3.7 \%$ also did not drastically affect rowing biomechanics. 
In summary, there were slight differences in the rowing biomechanics between oars of different stiffness and length. However, the differences in the biomechanical parameters were on the same order of magnitude as the rower's inter-stroke inconsistencies. It is important to note that the sample included national and world champion rowers. It is assumed that even greater inter-stroke inconsistencies in the biomechanical parameters would be observed in less experienced rowers. As this work focused on sculling oars, future research is still needed to investigate the biomechanics of rowing with sweep oar-shafts of different stiffness and length.

\subsection{References}

1. Affeld K, Schichl K and Ziemann A. Assessment of rowing efficiency. International Journal of Sports Medicine 1993; 14: 39-41.

2. Alexander FH. The theory of rowing. Proceedings of the University of Durham Philosophical Society 1925, pp.160-179.

3. Baudouin A and Hawkins D. A biomechanical review of factors affecting rowing performance. British Journal of Sports Medicine 2002; 36: 396-402.

4. Brearley $\mathrm{MN}$ and De Mestre NJ. Modeling the rowing stroke and increasing its efficiency. In: The third Conference on Mathematics and Computers in Sport, Bond University, Queensland, Australia, 30 Sept-2 Oct 1996, pp.35-46.

5. Cabrera D, Ruina A and Kleshnev V. A Simple 1+ Dimensional Model of Rowing Mimics Observed Forces and Motions. Human Movement Science 2006; 25: 192-220.

6. Findlay M and Turnock SR. Mechanics of a rowing stroke: Surge speed variations of a single scull. Proceedings of the Institution of Mechanical Engineers, Part P: Journal of Sports Engineering and Technology 2010; 224: 89100. 
7. Hofmijster MJ, Landman EH, Smith RM and Van Soest AJ. Effect of stroke rate on the distribution of net mechanical power in rowing. Journal of Sports Sciences 2007; 25: 403-411.

8. Macrossan MN and Macrossan NW. Energy efficiency of the rowing oar from catch to square-off. Report, University of Queensland, Australia, May 2008.

9. Sanderson B and Martindale W. Towards optimizing rowing technique. Medicine and Science in Sports and Exercise 1986; 18: 454-468.

10. Serveto S, Barre S, Kobus JM and Mariot JP. A three-dimensional model of the boat-oars-rower system using ADAMS and LifeMOD commercial software. Proceedings of the Institution of Mechanical Engineers, Part P: Journal of Sports Engineering and Technology 2010; 224: 75-88.

11. Zatsiorsky VM and Yakunin N. Mechanics and biomechanics of rowing: $A$ review. International Journal of Sport Biomechanics 1991; 7: 229-281.

12. Kleshnev V. Trampoline effect. Rowing Biomechanics Newsletter 2006; 6: 1.

13. Nolte V. Shorter Oars Are More Effective. Journal of Applied Biomechanics 2009; 25: 1-8.

14. Hofmijster M, De Koning J and Van Soest AJ. Estimation of the energy loss at the blades in rowing: Common assumptions revisited. Journal of Sports Science 2010; 28: 1093-1102.

15. Kinoshita T, Miyashita M, Kobayashi $\mathrm{H}$ and Hino T. Rowing Velocity Prediction Program with Estimating Hydrodynamic Load Acting on an Oar Blade. In: Kato $\mathrm{N}$ and Kamimura $\mathrm{S}$ (eds) Bio-mechanisms of Swimming and Flying: Fluid Dynamics, Biomimetic Robots, and Sports Science. Japan: Springer, 2008, pp.345-359. 
16. Sliasas $A$ and Tullis $S$. Modeling the effect of oar shaft bending during the rowing stroke. Proceedings of the Institution of Mechanical Engineers Part P Journal of Sports Engineering and Technology 2011; 225: 265-270.

17. Kleshnev $V$ and Nolte $V$. Changing oar length a few $\mathrm{cm}$ does not dramatically affects rowing biomechanics. Rowing Biomechanics Newsletter 2011; 11: 1.

18. Nielsen-Kellerman. Instruments, http://www.nkhome.com/kestrelproducts/instruments (accessed 30 June 2014).

19. Tenenbaum G and Driscoll MP. Methods of Research in Sport Sciences. UK: Meyer \& Meyer Sport, 2005, p.246.

20. Gust JC, Graham RB and Lombardi MA. Stopwatch and Timer Calibrations. National Institute of Standards and Technology Recommended Practice Guide, 2009, Special Publication 960-12.

21. Haines P. Force-sensing system. Patent 7114398 B2, USA, 2006.

22. Peach Innovations. PowerLine Rowing Instrumentation System, www.peachinnovations.com (assessed 11 June 2014).

23. McBride M. Rowing Biomechanics. In: Nolte V (ed) Rowing Faster. USA: Human Kinetics, 2005, pp.111-123.

24. Kleshnev V. Stroke rate and DPS of the medalists of the Olympic games 2004 in Athens. Rowing Biomechanics Newsletter 2005; 5: 1.

25. Kleshnev V. Case study: biomechanical parameters and rowing efficiency. Importance of fast force increasing. Rowing Biomechanics Newsletter 2004; 4: 1.

26. Kleshnev V. Timing of the stroke cycle and micro-phases at different patterns of force curve and various stroke rates. Importance of fast force increasing up to $70 \%$ of the maximum. Rowing Biomechanics Newsletter 2004; 4: 1. 
27. Kleshnev V and Kleshnev I. Dependence of rowing performance and efficiency on motor coordination of the main body segments. Journal of Sports Sciences 1998; 16: 418-419.

28. Millward A. A study of the forces exerted by an oarsmen and the effect on boat speed. Journal of Sports Sciences 1987; 5: 93-103.

29. Coker J, Hume PA and Nolte V. Evaluating rowing force profiles: implications from literature. Report, Auckland University of Technology, New Zealand, 2008. 


\section{Chapter 5}

\section{General Summary}

The mechanical properties of oar-shafts of different stiffness and length were investigated in Chapter 2. Static forces were applied to the oars at the junction between the shaft and the blade, and deflections were measured at several positions along the shafts. The flexural rigidity at each position was calculated by treating the oar-shafts as end-loaded cantilever beams. The results show that the oar-shafts are most compliant near the sleeves and become progressively stiffer towards the blades. The deflection angle was calculated from the tangent between the deflections at the two positions on the oar-shaft nearest to the blade. Differences in the deflection angles between the oar configurations have implications for on-water rowing because they may result in different blade angle of attacks for a given oar angle, and thus lead to different hydrodynamic forces on the blades. However, the maximum differences in the deflection angle between the oar configurations were less than the inter-stroke inconsistencies in the angular displacement of the oar for an elite sculling rower (Canadian Sport Institute Ontario, 2014, personal communication).

Chapter 3 investigated the convergent validity and test-retest reliability of the PowerLine Rowing Instrumentation System. The PowerLine system measures force on the oarlock using strain gauge load cells. The PowerLine oarlocks were secured to a horizontally oriented bar, and static forces were applied to each PowerLine oarlock via a suspension rig that was loaded with known weights; this test was repeated over multiple days. The differences between the PowerLine force measurements and the known static forces were statistically analyzed. The PowerLine force measurements were consistent over multiple days of testing, but were slightly less than the values of the known static forces. Calibration factors for each PowerLine oarlock were established to correct for the discrepancies in the force measurements. The maximum difference 
between the PowerLine force measurements and the known static forces was on the same order of magnitude as the inter-stroke inconsistencies in the maximum oarlock forces measured in elite rowers (Canadian Sport Institute Ontario, 2014, personal communication).

Chapter 4 investigated the biomechanics of rowing with oar-shafts of different stiffness and length. Single sculling boats were instrumented with the PowerLine oarlocks and accelerometers. Rowers rowed multiple trials over 200 $\mathrm{m}$ in the instrumented boats using the various oar configurations previously investigated in Chapter 2. There were small differences in the boat accelerations between the oars of different stiffness. The differences in acceleration were on the same order of magnitude as the rower's inter-stroke inconsistencies. There were no consistent intra or inter-rower trends in the differences in oarlock force between the oars of different stiffness. In terms of oar length, oars of $2.66 \mathrm{~m}$ consistently showed higher maximum forces, faster rates of force development to maximum force, and larger impulses on the oarlocks than oars of $2.70 \mathrm{~m}$. However, the differences in the biomechanical parameters between the oars of different lengths were less than the rower's inter-stroke inconsistencies. The sample included national and world champion rowers. It is projected that even greater inter-stroke inconsistencies in the biomechanical parameters would be observed in less experienced rowers. 


\section{Appendix}

\section{Appendix 1. Ethics Approval Notice from the University of Western Ontario Research Ethics Board for Health Sciences Research involving Human Subjects.}

\begin{tabular}{|c|c|c|}
\hline $\begin{array}{l}\text { Principal Imvestigator: Dr. Vokes Nolle } \\
\text { File Number:103737 } \\
\text { Review Level:Delegated } \\
\text { Approved Local Adult Participants: } 8 \\
\text { Approved Local Minor Participants:0 } \\
\text { Protocol Titie:The influence of oar-sha } \\
\text { Department \& Institution:Heath Sciem } \\
\text { Spensor: } \\
\text { Ethics Approval Date:June 24, } 2013 \text { E } \\
\text { Decuments Reviewed \& Approved \& }\end{array}$ & $\begin{array}{l}\text { At stifliness and length on scullin } \\
\text { cosikinesiology, Westem Unive } \\
\text { xpiry Date:July } 31,2014 \\
\text { Documents Received for Info }\end{array}$ & $\begin{array}{l}\text { g biomechanics } \\
\text { rsity } \\
\text { rmation: }\end{array}$ \\
\hline Document Name & Comments & Version Date \\
\hline Western University Protocol & & $2013 / 04 / 15$ \\
\hline Letter of Information & Letter of Information & $2013 / 06 / 19$ \\
\hline Letter of Information & Consent Form & $2013 / 06 / 19$ \\
\hline Other & Figures & $2013 / 06 / 19$ \\
\hline Instruments & PARQ & $2013 / 06 / 19$ \\
\hline
\end{tabular}

This is to notify you that The University of Western Ortario Research Ethics Boasd for Health Sciences Resescch Imvolving Human Subjects (HSREB) which is organized and operates acooeding to the Tri-Council Policy Statement. Ethical Conduct of Research and regulations of Ontario has reviewed and granted approval to the above referenced revision(s) or amendmentels) on the appeoval cate noted above. The membership of this REB also complies w th the membership requiements for REB's as defined in Division 5 of the Food and Drug Regulations.

The ethics approval for this study shall temain valid until the expiry date noted above assuming timely and acceptable responses to the HSREB's periodic requests for survellance and montoring irfomation. If you require an updated aporoval notice prior to that time you must request it using the University of Western Ontario Updated Agproval Request Form.

Members of the HSREB who are named as investigators in research studies, or declare a conflict of interest, do not participate in discussion related to, nor vote on, such studies when they are presented to the HSREB

The Chair of the HSREB is Dr. Joseph Gibert. The HSREB is registered with the U.S. Department of Health 8 Human Services under the IFB registration number IRB 00000940 .

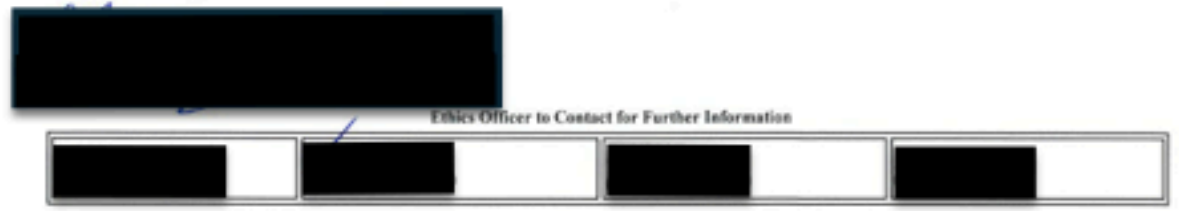

This is as oflocial document Please retain the onginal in your fless 


\section{Curriculum Vitae}

\section{Alma Mater}

- Master of Science, School of Kinesiology, University of Western Ontario (2014)

- Honours Bachelor, Faculty of Physical \& Health Education, University of

Toronto (2012)

- Summer Abroad Program, Masaryk University, Czech Republic (2008)

\section{Academic Publications}

- Laschowski, B. (2014). Technology in Rowing. International Sports Engineering Newsletter, 4-5.

- Laschowski, B. (2013). Oar Engineering. International Sports Engineering Newsletter, 1(3), 7.

- Laschowski, B. (2013). The Physics of Rowing. Phys13news, 145, 12-14.

\section{Conference Publications}

- Rupf, R., Schultz, L., Musalem, L., Laschowski, B., Fernandes, E., \& Fischer, HC. (2013). Poling rates in female cross country skiers using different techniques. Canadian Society for Exercise Physiology Annual General Meeting, Toronto, Canada.

- Laschowski, B., Zang., Y., Nolte, V., \& George, W. (2013). Statistical Analysis of the PowerLine Rowing Instrumentation System. Sport Innovation Summit, Calgary, Canada.

- Laschowski, B., Nolte, V., \& de Bruyn, J. (2013). The influence of oar-shaft stiffness \& length on rowing biomechanics. Canadian Association of Physicists Congress, Montreal, Canada.

- Laschowski, B., \& Nolte, V. (2013). The influence of oar-shaft stiffness \& length on rowing biomechanics. 10th Annual Ontario Biomechanics Conference, Barrie, Canada.

- Laschowski, B., \& Taha, T. (2012). The effects of explosive strength training on 
jump biomechanics. 20th International Congress on Sports Sciences for Students, Budapest, Hungary.

- Laschowski, B., \& Taha, T. (2012). The effects of detraining on elite jump athletes. 13th Bertha Rosenstadt National Undergraduate Research Conference in Kinesiology, Toronto, Canada.

\section{Academic Service}

- University of Western Ontario, Graduate Teaching Assistant (2012-2014)

- Biomechanical Analysis of Human Location, School of Kinesiology

- Introduction to Athletic Injuries, School of Kinesiology

- Physiology of Fitness Appraisal, School of Kinesiology

- University of Western Ontario, Graduate Student Ambassador (2013)

- Faculty of Health Sciences

- University of Western Ontario, Exam Proctor (2013)

- Anatomy of the Human Body, Department of Anatomy and Cell Biology

- Introductory Biomechanics, School of Kinesiology

- University of Western Ontario, Graduate Research Assistant (2012-2014)

- The Richard \& Jean Ivey Sport Biomechanics Lab, School of Kinesiology

- University of Toronto, Undergraduate Research Assistant (2010-2012)

- Faculty of Physical and Health Education

\section{Professional Service}

- Journal of Sports Engineering \& Technology, Reviewer (2014)

- Canadian Sport Institute Ontario, Biomechanics Intern (2011-2014)

- Olympic \& National Teams: Trampoline / Beach Volleyball / Wheelchair

Basketball / Women's Ice Hockey / Swimming / Cross-Country Skiing / Women's Heavyweight Rowing / Men's Lightweight Rowing / Under 23 Women's Heavyweight Rowing

\section{Awards \& Scholarships}


- International Sport Engineering Association, Student Project Semi-Finalist (2013)

- Canadian Association of Physicists Congress, 1st Prize \& Best Presentation (2013)

- University of Western Ontario, Graduate Research Scholarship (2012-2014)

- University of Western Ontario, Faculty of Health Science Travel Award (20122014)

- University of Western Ontario, Kinesiology Travel Award (2013)

- University of Toronto, Peter Klavora Research Award (2012)

\section{Membership}

- Canadian Society for Biomechanics

- International Sports Engineering Association

- International Society of Biomechanics in Sports

\section{Media Appearances}

- Laschowski, B. Sports Technology. Interviewed by Dr. Mike Vasquez \& Henry Hanson [Online Podcast]. Recorded December 19, 2013.

- Laschowski, B. Gradcast. Interviewed by Chris Vandenbreekel [Radio] 94.9 CHRW. Recorded November 12, 2013. 